\title{
CMS tracking performance results from early LHC operation
}

\author{
The CMS Collaboration
}

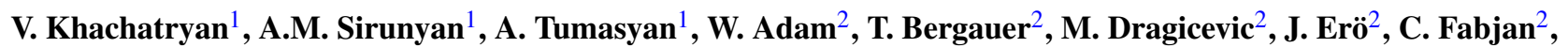
M. Friedl' ${ }^{2}$, R. Frühwirth ${ }^{2}$, V.M. Ghete ${ }^{2}$, J. Hammer ${ }^{2, b}$, S. Hänsel ${ }^{2}$, M. Hoch ${ }^{2}$, N. Hörmann ${ }^{2}$, J. Hrubec ${ }^{2}$, M. Jeitler ${ }^{2}$, G. Kasieczka² ${ }^{2}$ W. Kiesenhofer ${ }^{2}$, M. Krammer ${ }^{2}$, D. Liko ${ }^{2}$, I. Mikulec ${ }^{2}$, M. Pernicka ${ }^{2}$, H. Rohringer ${ }^{2}$, R. Schöfbeck ${ }^{2}$, J. Strauss ${ }^{2}$, A. Taurok ${ }^{2}$, F. Teischinger ${ }^{2}$, W. Waltenberger ${ }^{2}$, G. Walzel ${ }^{2}$, E. Widl ${ }^{2}$, C.-E. Wulz ${ }^{2}$, V. Mossolov ${ }^{3}$, N. Shumeiko ${ }^{3}$, J. Suarez Gonzalez ${ }^{3}$, L. Benucci ${ }^{4}$, L. Ceard ${ }^{4}$, E.A. De Wolf ${ }^{4}$, X. Janssen ${ }^{4}$, T. Maes ${ }^{4}$, L. Mucibello ${ }^{4}$, S. Ochesanu ${ }^{4}$, B. Roland ${ }^{4}$, R. Rougny ${ }^{4}$, M. Selvaggi ${ }^{4}$, H. Van Haevermaet ${ }^{4}$, P. Van Mechelen ${ }^{4}$, N. Van Remortel ${ }^{4}$, V. Adler ${ }^{5}$, S. Beauceron ${ }^{5}$, S. Blyweert ${ }^{5}$, J. D'Hondt ${ }^{5}$, O. Devroede ${ }^{5}$, A. Kalogeropoulos ${ }^{5}$, J. Maes ${ }^{5}$, M. Maes ${ }^{5}$, S. Tavernier ${ }^{5}$, W. Van Doninck ${ }^{5}$, P. Van Mulders ${ }^{5}$, I. Villella ${ }^{5}$, E.C. Chabert ${ }^{6}$, O. Charaf $^{6}$, B. $_{\text {Clerbaux }}^{6}$, G. De Lentdecker ${ }^{6}$, V. Dero ${ }^{6}$, A.P.R. Gay ${ }^{6}$, G.H. Hammad ${ }^{6}$, P.E. Marage $^{6}$, C. Vander Velde $^{6}$, P. Vanlaer ${ }^{6}$, J. Wickens ${ }^{6}$, S. Costantini ${ }^{7}$, M. Grunewald ${ }^{7}$, B. Klein ${ }^{7}$, A. Marinov ${ }^{7}$, D. Ryckbosch ${ }^{7}$, F. Thyssen ${ }^{7}$, M. Tytgat ${ }^{7}$, L. Vanelderen ${ }^{7}$, P. Verwilligen ${ }^{7}$, S. Walsh ${ }^{7}$, N. Zaganidis ${ }^{7}$, S. Basegmez ${ }^{8}$, G. Bruno ${ }^{8}$, J. Caudron ${ }^{8}$, J. De Favereau De

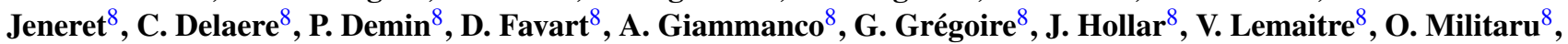
S. Ovyn ${ }^{8}$, D. Pagano ${ }^{8}$, A. Pin ${ }^{8}$, K. Piotrzkowski ${ }^{8, b}$, L. Quertenmont ${ }^{8}$, N. Schul ${ }^{8}$, N. Beliy ${ }^{9}$, T. Caebergs ${ }^{9}$, E. Daubie ${ }^{9}$, G.A. Alves ${ }^{10}$, M.E. Pol ${ }^{10}$, M.H.G. Souza ${ }^{10}$, W. Carvalho ${ }^{11}$, E.M. Da Costa ${ }^{11}$, D. De Jesus Damiao ${ }^{11}$, C. De Oliveira Martins ${ }^{11}$, S. Fonseca De Souza ${ }^{11}$, L. Mundim ${ }^{11}$, H. Nogima ${ }^{11}$, V. Oguri ${ }^{11}$, A. Santoro ${ }^{11}$, S.M. Silva Do Amaral ${ }^{11}$, A. Sznajder ${ }^{11}$, F. Torres Da Silva De Araujo ${ }^{11}$, F.A. Dias ${ }^{12}$, M.A.F. Dias ${ }^{12}$, T.R. Fernandez Perez Tomei ${ }^{12}$, E.M. Gregores ${ }^{12, c}$, F. Marinho ${ }^{12}$, S.F. Novaes ${ }^{12}$, Sandra S. Padula ${ }^{12}$, N. Darmenov ${ }^{13, b}$, L. Dimitrov ${ }^{13}$, V. Genchev ${ }^{13, b}$, P. Iaydjiev ${ }^{13, b}$, S. Piperov ${ }^{13}$, S. Stoykova ${ }^{13}$, G. Sultanov ${ }^{13}$, R. Trayanov ${ }^{13}$, I. Vankov ${ }^{13}$, M. Dyulendarova ${ }^{14}$, R. Hadjiiska ${ }^{14}$, V. Kozhuharov ${ }^{14}$, L. Litov ${ }^{14}$, E. Marinova ${ }^{14}$, M. Mateev ${ }^{14, \dagger}$, B. Pavlov ${ }^{14}$, P. Petkov ${ }^{14}$, J.G. Bian ${ }^{15}$, G.M. Chen ${ }^{15}$, H.S. Chen ${ }^{15}$, C.H. Jiang ${ }^{15}$, D. Liang ${ }^{15}$, S. Liang ${ }^{15}$, J. Wang ${ }^{15}$, J. Wang ${ }^{15}$, X. Wang ${ }^{15}$, Z. Wang ${ }^{15}$, M. Yang ${ }^{15}$, J. Zang ${ }^{15}$, Z. Zhang ${ }^{15}$, Y. Ban ${ }^{16}$, S. Guo ${ }^{16}$, Z. Hu ${ }^{16}$, Y. Mao $^{16}$, S.J. Qian ${ }^{16}$, H. Teng ${ }^{16}$, B. Zhu ${ }^{16}$, A. Cabrera ${ }^{17}$, C.A. Carrillo Montoya ${ }^{17}$, B. Gomez Moreno ${ }^{17}$,

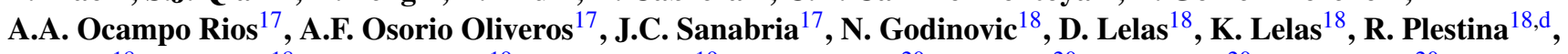
D. Polic ${ }^{18}$, I. Puljak ${ }^{18}$, Z. Antunovic ${ }^{19}$, M. Dzelalija ${ }^{19}$, V. Brigljevic ${ }^{20}$, S. Duric $^{20}$, K. Kadija ${ }^{20}$, S. Morovic ${ }^{20}$,

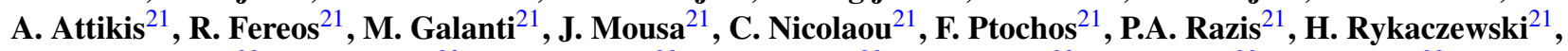
M.A. Mahmoud ${ }^{22, \text { e }}$, A. Hektor ${ }^{23}$, M. Kadastik ${ }^{23}$, K. Kannike ${ }^{23}$, M. Müntel ${ }^{23}$, M. Raidal ${ }^{23}$, L. Rebane ${ }^{23}$, V. Azzolini ${ }^{24}$, P. Eerola ${ }^{24}$, S. Czellar ${ }^{25}$, J. Härkönen ${ }^{25}$, A. Heikkinen ${ }^{25}$, V. Karimäki ${ }^{25}$, R. Kinnunen ${ }^{25}$, J. Klem ${ }^{25}$, M.J. Kortelainen ${ }^{25}$, T. Lampén ${ }^{25}$, K. Lassila-Perini2 ${ }^{25}$, S. Lehti ${ }^{25}$, T. Lindén ${ }^{25}$, P. Luukka ${ }^{25}$, T. Mäenpää25, S. Sarkar ${ }^{25}$, E. Tuominen ${ }^{25}$, J. Tuominiemi ${ }^{25}$, E. Tuovinen ${ }^{25}$, D. Ungaro ${ }^{25}$, L. Wendland ${ }^{25}$, K. Banzuzi ${ }^{26}$, A. Korpela ${ }^{26}$, T. Tuuva ${ }^{26}$, D. Sillou ${ }^{27}$, M. Besancon ${ }^{28}$, M. Dejardin ${ }^{28}$, D. Denegri $^{28}$, J. Descamps $^{28}$, B. Fabbro ${ }^{28}$, J.L. Faure ${ }^{28}$, F. Ferri ${ }^{28}$, S. Ganjour ${ }^{28}$, F.X. Gentit ${ }^{28}$, A. Givernaud ${ }^{28}$, P. Gras ${ }^{28}$, G. Hamel de Monchenault ${ }^{28}$,

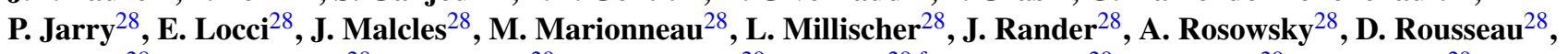
M. Titov ${ }^{28}$, P. Verrecchia ${ }^{28}$, S. Baffioni ${ }^{29}$, L. Bianchini ${ }^{29}$, M. Bluj ${ }^{29, f}$, C. Broutin ${ }^{29}$, P. Busson ${ }^{29}$, C. Charlot $^{29}$, L. Dobrzynski ${ }^{29}$, S. Elgammal ${ }^{29}$, R. Granier de Cassagnac $^{29}$, M. Haguenauer ${ }^{29}$, A. Kalinowski ${ }^{29}$, P. Miné ${ }^{29}$,

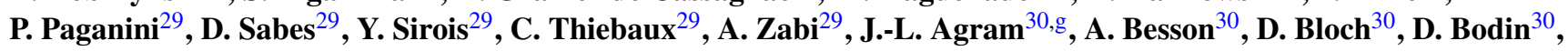

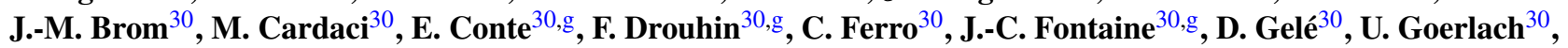

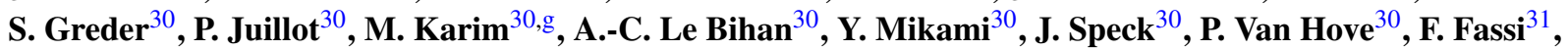

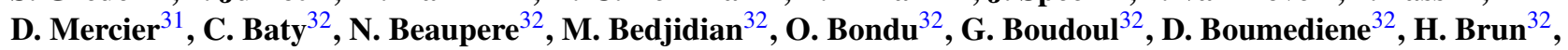

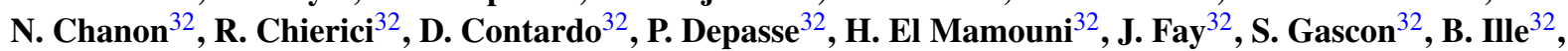

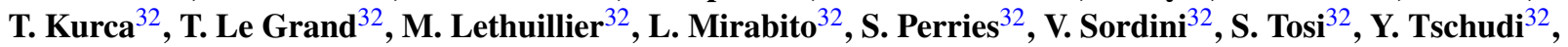

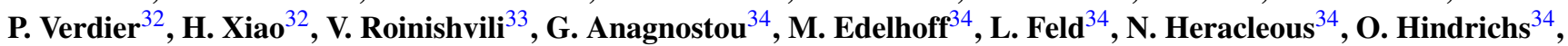
R. Jussen ${ }^{34}$, K. Klein ${ }^{34}$, J. Merz ${ }^{34}$, N. Mohr ${ }^{34}$, A. Ostapchuk ${ }^{34}$, A. Perieanu ${ }^{34}$, F. Raupach ${ }^{34}$, J. Sammet ${ }^{34}$, S. Schael ${ }^{34}$, D. Sprenger ${ }^{34}$, H. Weber ${ }^{34}$, M. Weber ${ }^{34}$, B. Wittmer ${ }^{34}$, O. Actis ${ }^{35}$, M. Ata ${ }^{35}$, W. Bender ${ }^{35}$, P. Biallass ${ }^{35}$, M. Erdmann ${ }^{35}$, J. Frangenheim ${ }^{35}$, T. Hebbeker ${ }^{35}$, A. Hinzmann ${ }^{35}$, K. Hoepfner ${ }^{35}$, C. Hof ${ }^{35}$, M. Kirsch ${ }^{35}$, 
T. Klimkovich ${ }^{35}$, P. Kreuzer ${ }^{35, b}$, D. Lanske ${ }^{35, \dagger}$, C. Magass ${ }^{35}$, M. Merschmeyer ${ }^{35}$, A. Meyer ${ }^{35}$, P. Papacz ${ }^{35}$, H. Pieta ${ }^{35}$, H. Reithler ${ }^{35}$, S.A. Schmitz ${ }^{35}$, L. Sonnenschein ${ }^{35}$, M. Sowa ${ }^{35}$, J. Steggemann ${ }^{35}$, D. Teyssier ${ }^{35}$, C. Zeidler ${ }^{35}$, M. Bontenackels ${ }^{36}$, M. Davids ${ }^{36}$, M. Duda ${ }^{36}$, G. Flügge ${ }^{36}$, H. Geenen ${ }^{36}$, M. Giffels ${ }^{36}$, W. Haj Ahmad ${ }^{36}$,

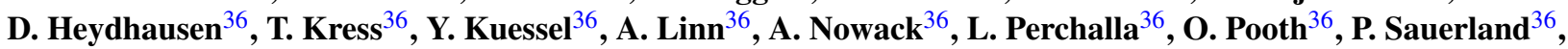
A. Stahl ${ }^{36}$, M. Thomas ${ }^{36}$, D. Tornier ${ }^{36}$, M.H. Zoeller ${ }^{36}$, M. Aldaya Martin ${ }^{37}$, W. Behrenhoff ${ }^{37}$, U. Behrens ${ }^{37}$, M. Bergholz ${ }^{37}$, K. Borras ${ }^{37}$, A. Campbell ${ }^{37}$, E. Castro ${ }^{37}$, D. Dammann ${ }^{37}$, G. Eckerlin ${ }^{37}$, A. Flossdorf ${ }^{37}$, G. Flucke ${ }^{37}$,

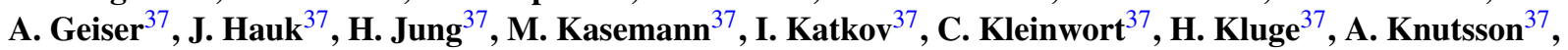

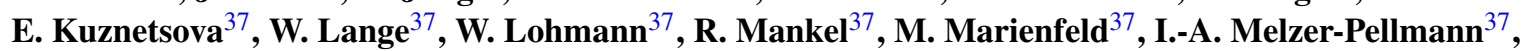
A.B. Meyer $^{37}$, J. Mnich $^{37}$, A. Mussgiller ${ }^{37}$, J. Olzem ${ }^{37}$, A. Parenti ${ }^{37}$, A. Raspereza ${ }^{37}$, R. Schmidt ${ }^{37}$, T. Schoerner-Sadenius ${ }^{37}$, N. Sen ${ }^{37}$, M. Stein ${ }^{37}$, J. Tomaszewska ${ }^{37}$, D. Volyanskyy ${ }^{37}$, C. Wissing ${ }^{37}$, C. Autermann ${ }^{38}$, S. Bobrovskyi ${ }^{38}$, J. Draeger ${ }^{38}$, D. Eckstein ${ }^{38}$, H. Enderle ${ }^{38}$, U. Gebbert ${ }^{38}$, K. Kaschube ${ }^{38}$, G. Kaussen ${ }^{38}$, R. Klanner ${ }^{38}$, B. Mura ${ }^{38}$, S. Naumann-Emme ${ }^{38}$, F. Nowak ${ }^{38}$, N. Pietsch ${ }^{38}$, C. Sander ${ }^{38}$, H. Schettler ${ }^{38}$, P. Schleper ${ }^{38}$, M. Schröder ${ }^{38}$, T. Schum ${ }^{38}$, J. Schwandt ${ }^{38}$, A.K. Srivastava ${ }^{38}$, H. Stadie ${ }^{38}$, G. Steinbrück ${ }^{38}$, J. Thomsen ${ }^{38}$,

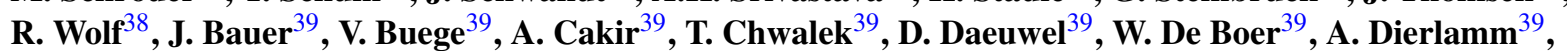
G. Dirkes $^{39}$, M. Feindt ${ }^{39}$, J. Gruschke ${ }^{39}$, C. Hackstein ${ }^{39}$, F. Hartmann ${ }^{39}$, M. Heinrich ${ }^{39}$, H. Held ${ }^{39}$, K.H. Hoffmann ${ }^{39}$, S. Honc ${ }^{39}$, T. Kuhr ${ }^{39}$, D. Martschei ${ }^{39}$, S. Mueller ${ }^{39}$, Th. Müller ${ }^{39}$, M. Niegel ${ }^{39}$, O. Oberst $^{39}$,

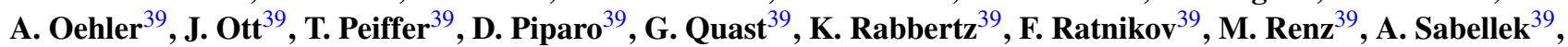
C. Saout ${ }^{39}$, A. Scheurer ${ }^{39}$, P. Schieferdecker ${ }^{39}$, F.-P. Schilling ${ }^{39}$, G. Schott ${ }^{39}$, H.J. Simonis ${ }^{39}$, F.M. Stober ${ }^{39}$, D. Troendle ${ }^{39}$, J. Wagner-Kuhr ${ }^{39}$, M. Zeise ${ }^{39}$, V. Zhukov ${ }^{39, h}$, E.B. Ziebarth ${ }^{39}$, G. Daskalakis ${ }^{40}$, T. Geralis ${ }^{40}$,

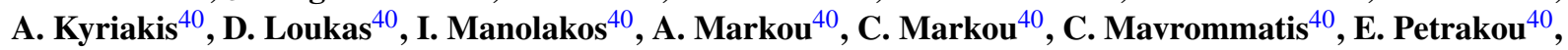

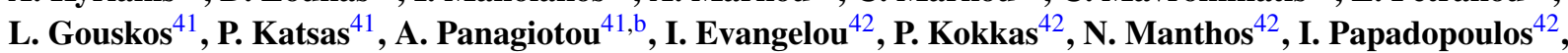
V. Patras ${ }^{42}$, F.A. Triantis ${ }^{42}$, A. Aranyi ${ }^{43}$, G. Bencze ${ }^{43}$, L. Boldizsar ${ }^{43}$, G. Debreczeni ${ }^{43}$, C. Hajdu ${ }^{43, b}$, D. Horvath ${ }^{43, i}$, A. Kapusii ${ }^{43}$, K. Krajczar ${ }^{43, j}$, A. Laszlo ${ }^{43}$, F. Sikler ${ }^{43}$, G. Vesztergombi ${ }^{43, j}$, N. Beni ${ }^{44}$, J. Molnar ${ }^{44}$, J. Palinkas ${ }^{44}$, Z. Szillasi ${ }^{44, b}$, V. Veszpremi ${ }^{44}$, P. Raics ${ }^{45}$, Z.L. Trocsanyi ${ }^{45}$, B. Ujvari ${ }^{45}$, S. Bansal ${ }^{46}$, S.B. Beri ${ }^{46}$, V. Bhatnagar ${ }^{46}$, M. Jindal ${ }^{46}$, M. Kaur ${ }^{46}$, J.M. Kohli ${ }^{46}$, M.Z. Mehta ${ }^{46}$, N. Nishu ${ }^{46}$, L.K. Saini ${ }^{46}$, A. Sharma ${ }^{46}$, R. Sharma ${ }^{46}$, A.P. Singh ${ }^{46}$, J.B. Singh ${ }^{46}$, S.P. Singh ${ }^{46}$, S. Ahuja ${ }^{47}$, S. Bhattacharya ${ }^{47}$, S. Chauhan ${ }^{47}$, B.C. Choudhary ${ }^{47}$, P. Gupta ${ }^{47}$, S. Jain ${ }^{47}$, S. Jain ${ }^{47}$, A. Kumar ${ }^{47}$, K. Ranjan ${ }^{47}$, R.K. Shivpuri ${ }^{47}$, R.K. Choudhury ${ }^{48}$, D. Dutta ${ }^{48}$, S. Kailas ${ }^{48}$, S.K. Kataria ${ }^{48}$, A.K. Mohanty ${ }^{48}$, L.M. Pant ${ }^{48}$, P. Shukla ${ }^{48}$, P. Suggisetti ${ }^{48}$, T. Aziz ${ }^{49}$, M. Guchait ${ }^{49, k}$, A. Gurtu ${ }^{49}$, M. Maity ${ }^{49,1}$, D. Majumder $^{49}$, G. Majumder $^{49}$, K. Mazumdar $^{49}$, G.B. Mohanty ${ }^{49}$, A. Saha ${ }^{49}$, K. Sudhakar ${ }^{49}$,

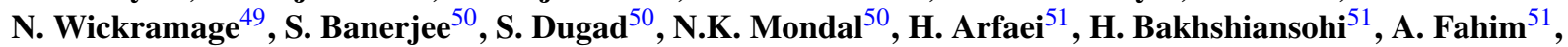
M. Hashemi ${ }^{51}$, A. Jafari ${ }^{51}$, M. Mohammadi Najafabadi ${ }^{51}$, S. Paktinat Mehdiabadi ${ }^{51}$, B. Safarzadeh ${ }^{51}$, M. Zeinali ${ }^{51}$, M. Abbrescia ${ }^{52,53}$, L. Barbone ${ }^{52}$, A. Colaleo ${ }^{52}$, D. Creanza ${ }^{52,54}$, N. De Filippis ${ }^{52}$, M. De Palma ${ }^{52,53}$, A. Dimitrov . $^{52}$,

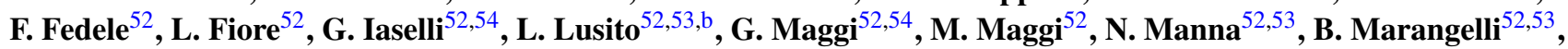

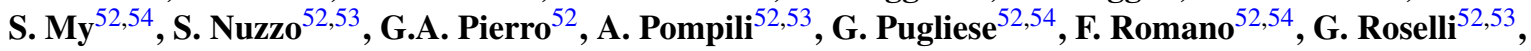
G. Selvaggi ${ }^{52,53}$, L. Silvestris ${ }^{52}$, R. Trentadue ${ }^{52}$, S. Tupputi ${ }^{52,53}$, G. Zito $^{52}$, G. Abbiendi ${ }^{55}$, A.C. Benvenuti ${ }^{55}$, D. Bonacorsi ${ }^{55}$, S. Braibant-Giacomelli ${ }^{55,56}$, P. Capiluppi ${ }^{55,56}$, A. Castro ${ }^{55,56}$, F.R. Cavallo ${ }^{55}$, G. Codispoti ${ }^{55,56}$, M. Cuffiani ${ }^{55,56}$, G.M. Dallavalle ${ }^{55, b}$, F. Fabbri ${ }^{55}$, A. Fanfani ${ }^{55,56}$, D. Fasanella ${ }^{55}$, P. Giacomelli ${ }^{55}$, M. Giunta ${ }^{55, b}$, S. Marcellini ${ }^{55}$, G. Masetti ${ }^{55,56}$, A. Montanari ${ }^{55}$, F.L. Navarria ${ }^{55,56}$, F. Odorici ${ }^{55}$, A. Perrotta ${ }^{55}$, A.M. Rossi ${ }^{55,56}$,

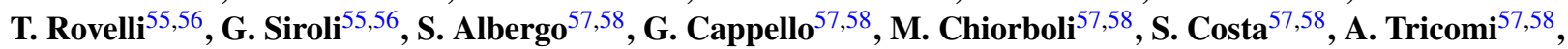
C. Tuve ${ }^{57}$, G. Barbagli ${ }^{59}$, G. Broccolo ${ }^{59,60}$, V. Ciulli ${ }^{59,60}$, C. Civinini ${ }^{59}$, R. D’Alessandro ${ }^{59,60}$, E. Focardi ${ }^{59,60}$,

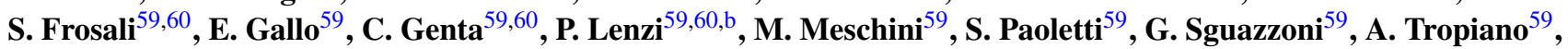

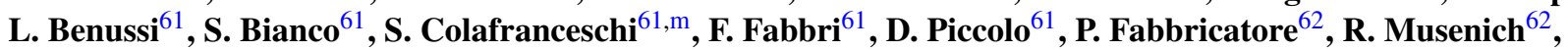
A. Benaglia ${ }^{63,64}$, G.B. Cerati ${ }^{63,64, b}$, F. De Guio ${ }^{63,64}$, L. Di Matteo ${ }^{63,64}$, A. Ghezzi ${ }^{63,64, b}$, P. Govoni ${ }^{63,64}$, M. Malberti ${ }^{63,64, b}$, S. Malvezzi $^{63}$, A. Martelli ${ }^{63,64, d}$, A. Massironi ${ }^{63,64}$, D. Menasce ${ }^{63}$, V. Miccio ${ }^{63,64}$, L. Moroni ${ }^{63}$, P. Negri ${ }^{63,64}$, M. Paganoni ${ }^{63,64}$, D. Pedrini ${ }^{63}$, S. Ragazzi ${ }^{63,64}$, N. Redaelli ${ }^{63}$, S. Sala ${ }^{63}$, R. Salerno ${ }^{63,64}$, T. Tabarelli de

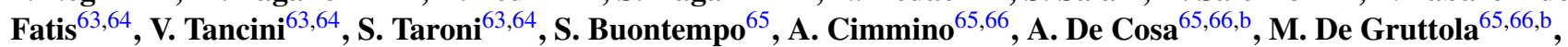

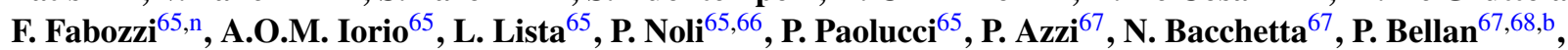
D. Bisello ${ }^{67,68}$, R. Carlin ${ }^{67,68}$, P. Checchia ${ }^{67}$, E. Conti ${ }^{67}$, M. De Mattia ${ }^{67,68}$, T. Dorigo ${ }^{67}$, U. Dosselli ${ }^{67}$, F. Fanzago ${ }^{67}$, F. Gasparini ${ }^{67,68}$, U. Gasparini ${ }^{67,68}$, P. Giubilato ${ }^{67,68}$, A. Gresele ${ }^{67,69}$, S. Lacaprara $^{67,0}$, I. Lazzizzera ${ }^{67,69}$, M. Margoni ${ }^{67,68}$, M. Mazzucato ${ }^{67}$, A.T. Meneguzzo ${ }^{67,68}$, L. Perrozzi ${ }^{67}$, N. Pozzobon ${ }^{67,68}$, P. Ronchese $^{67,68}$, F. Simonetto ${ }^{67,68}$, E. Torassa ${ }^{67}$, M. Tosi ${ }^{67,68}$, S. Vanini ${ }^{67,68}$, P. Zotto ${ }^{67,68}$, G. Zumerle ${ }^{67,68}$, P. Baesso ${ }^{70,71}$,

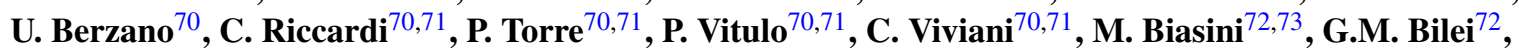


B. Caponeri ${ }^{72,73}$, L. Fanò ${ }^{72}$, P. Lariccia ${ }^{72,73}$, A. Lucaroni ${ }^{72,73}$, G. Mantovani ${ }^{72,73}$, M. Menichelli ${ }^{72}$, A. Nappi ${ }^{72,73}$, A. Santocchia ${ }^{72,73}$, L. Servoli ${ }^{72}$, M. Valdata ${ }^{72}$, R. Volpe .2,73,b $^{72}$ P. Azzurri ${ }^{74,76}$, G. Bagliesi ${ }^{74}$, J. Bernardini ${ }^{74,75, b}$, T. Boccali ${ }^{74, b}$, R. Castaldi ${ }^{74}$, R.T. Dagnolo ${ }^{74,76}$, R. Dell'Orso ${ }^{74}$, F. Fiori ${ }^{74,75}$, L. Foà ${ }^{74,76}$, A. Giassii ${ }^{74}$, A. Kraan ${ }^{74}$,

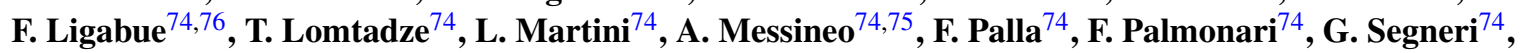

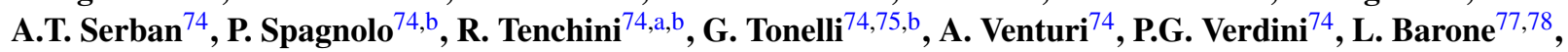

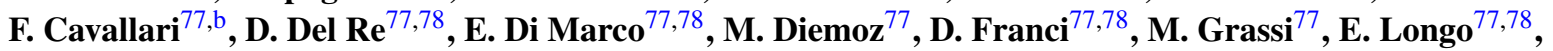

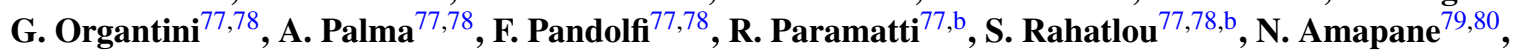
R. Arcidiacono ${ }^{79,80}$, S. Argiro ${ }^{79,80}$, M. Arneodo ${ }^{79,81}$, C. Biino $^{79}$, C. Botta $^{79,80}$, N. Cartiglia $^{79}$, R. Castello ${ }^{79,80}$, M. Costa ${ }^{79,80}$, N. Demaria ${ }^{79}$, A. Graziano ${ }^{79,80}$, C. Mariotti ${ }^{79}$, M. Marone $^{79,80}$, S. Maselli ${ }^{79}$, E. Migliore ${ }^{79,80}$, G. Mila ${ }^{79,80}$, V. Monaco ${ }^{79,80}$, M. Musich ${ }^{79,80}$, M.M. Obertino ${ }^{79,81}$, N. Pastrone ${ }^{79}$, M. Pelliccioni ${ }^{79,80, b}$, A. Romero ${ }^{79,80}$, M. Ruspa ${ }^{79,81}$, R. Sacchi ${ }^{79,80}$, A. Solano ${ }^{79,80}$, A. Staiano ${ }^{79}$, D. Trocino ${ }^{79,80}$, A. Vilela Pereira ${ }^{79,80, b}$, F. Ambroglini ${ }^{82,83}$, S. Belforte ${ }^{82}$, F. Cossutti ${ }^{82}$, G. Della Ricca ${ }^{82,83}$, B. Gobbo ${ }^{82}$, D. Montanino ${ }^{82}$, A. Penzo ${ }^{82}$, H. Kim ${ }^{84}$, S. Chang ${ }^{85}$, J. Chung ${ }^{85}$, D.H. Kim ${ }^{85}$, G.N. Kim ${ }^{85}$, J.E. Kim ${ }^{85}$, D.J. Kong ${ }^{85}$, H. Park ${ }^{85}$, D. Son ${ }^{85}$, D.C. Son ${ }^{85}$, Z. Kim ${ }^{86}$, J.Y. Kim ${ }^{86}$, S. Song ${ }^{86}$, S. Choi ${ }^{87}$, B. Hong ${ }^{87}$, H. Kim ${ }^{87}$, J.H. Kim ${ }^{87}$, T.J. Kim ${ }^{87}$, K.S. Lee $^{87}$, D.H. Moon ${ }^{87}$, S.K. Park ${ }^{87}$,

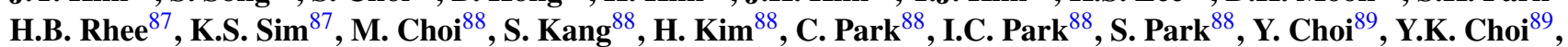
J. Goh ${ }^{89}$, J. Lee ${ }^{89}$, S. Lee ${ }^{89}$, H. Seo ${ }^{89}$, I. Yu ${ }^{89}$, M. Janulis ${ }^{90}$, D. Martisiute ${ }^{90}$, P. Petrov ${ }^{90}$, T. Sabonis ${ }^{90}$, S. Carrillo Moreno ${ }^{91}$, H.A. Salazar Ibarguen ${ }^{92}$, E. Casimiro Linares ${ }^{93}$, A. Morelos Pineda ${ }^{93}$, M.A. Reyes-Santos ${ }^{93}$, P. Allfrey ${ }^{94}$, D. Krofcheck ${ }^{94}$, J. Tam ${ }^{94}$, P.H. Butler ${ }^{95}$, T. Signal ${ }^{95}$, J.C. Williams ${ }^{95}$, M. Ahmad ${ }^{96}$, I. Ahmed ${ }^{96}$, M.I. Asghar ${ }^{96}$, H.R. Hoorani ${ }^{96}$, W.A. Khan ${ }^{96}$, T. Khurshid ${ }^{96}$, S. Qazi ${ }^{96}$, M. Cwiok ${ }^{97}$, W. Dominik ${ }^{97}$, K. Doroba ${ }^{97}$, M. Konecki ${ }^{97}$, J. Krolikowski ${ }^{97}$, T. Frueboes ${ }^{98}$, R. Gokieli ${ }^{98}$, M. Górski ${ }^{98}$, M. Kazana ${ }^{98}$, K. Nawrocki ${ }^{98}$, M. Szleper ${ }^{98}$, G. Wrochna ${ }^{98}$, P. Zalewski ${ }^{98}$, N. Almeida ${ }^{99}$, A. David ${ }^{99}$, P. Faccioli $^{99}$, P.G. Ferreira Parracho ${ }^{99}$, M. Gallinaro ${ }^{99}$, P. Martins ${ }^{99}$, G. Mini ${ }^{99}$, P. Musella ${ }^{99}$, A. Nayak ${ }^{99}$, L. Raposo ${ }^{99}$, P.Q. Ribeiro ${ }^{99}$, J. Seixas ${ }^{99}$, P. Silva ${ }^{99}$, D. Soares ${ }^{99}$, J. Varela ${ }^{99, b}$, H.K. Wöhri9 ${ }^{99}$, I. Belotelov ${ }^{100}$, P. Bunin ${ }^{100}$, M. Finger ${ }^{100}$, M. Finger Jr. ${ }^{100}$, I. Golutvin ${ }^{100}$, A. Kamenev ${ }^{100}$, V. Karjavin ${ }^{100}$, G. Kozlov ${ }^{100}$, A. Lanev ${ }^{100}$, P. Moisenz ${ }^{100}$, V. Palichik ${ }^{100}$, V. Perelygin ${ }^{100}$, S. Shmatov ${ }^{100}$, V. Smirnov ${ }^{100}$, A. Volodko ${ }^{100}$, A. Zarubin ${ }^{100}$, N. Bondar ${ }^{101}$, V. Golovtsov ${ }^{101}$, Y. Ivanov ${ }^{101}$, V. Kim ${ }^{101}$, P. Levchenko ${ }^{101}$, I. Smirnov ${ }^{101}$, V. Sulimov ${ }^{101}$, L. Uvarov ${ }^{101}$, S. Vavilov ${ }^{101}$, A. Vorobyev ${ }^{101}$, Yu. Andreev ${ }^{102}$, S. Gninenko ${ }^{102}$, N. Golubev ${ }^{102}$, M. Kirsanov ${ }^{102}$, N. Krasnikov ${ }^{102}$, V. Matveev ${ }^{102}$, A. Pashenkov ${ }^{102}$, A. Toropin ${ }^{102}$, S. Troitsky ${ }^{102}$, V. Epshteyn ${ }^{103}$, V. Gavrilov ${ }^{103}$, N. Ilina ${ }^{103}$, V. Kaftanov $^{103, \dagger}$, M. Kossov ${ }^{103, b}$, A. Krokhotin ${ }^{103}$, S. Kuleshov ${ }^{103}$, A. Oulianov ${ }^{103}$, G. Safronov ${ }^{103}$, S. Semenov ${ }^{103}$, I. Shreyber ${ }^{103}$, V. Stolin ${ }^{103}$, E. Vlasov ${ }^{103}$, A. Zhokin ${ }^{103}$, E. Boos ${ }^{104}$, M. Dubinin ${ }^{104, p}$, L. Dudko ${ }^{104}$, A. Ershov ${ }^{104}$, A. Gribushin ${ }^{104}$, O. Kodolova ${ }^{104}$, I. Lokhtin ${ }^{104}$, S. Obraztsov ${ }^{104}$, S. Petrushanko ${ }^{104}$, L. Sarycheva ${ }^{104}$, V. Savrin ${ }^{104}$, A. Snigirev ${ }^{104}$, V. Andreev ${ }^{105}$, I. Dremin ${ }^{105}$, M. Kirakosyan ${ }^{105}$, S.V. Rusakov ${ }^{105}$, A. Vinogradov ${ }^{105}$, I. Azhgirey ${ }^{106}$, S. Bitioukov ${ }^{106}$, K. Datsko ${ }^{106}$, V. Grishin ${ }^{106, b}$, V. Kachanov ${ }^{106}$, D. Konstantinov ${ }^{106}$, V. Krychkine ${ }^{106}$, V. Petrov ${ }^{106}$, R. Ryutin ${ }^{106}$, S. Slabospitsky ${ }^{106}$,

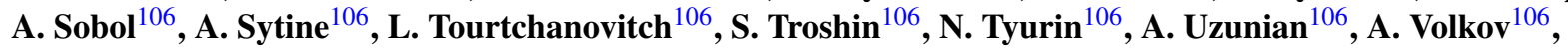

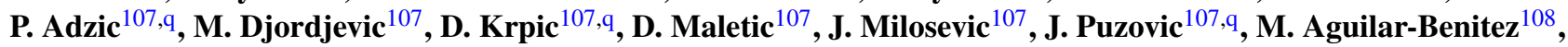

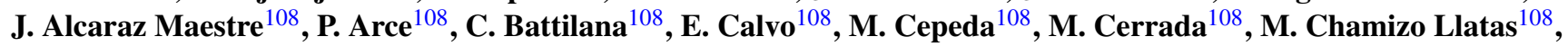
N. Colino ${ }^{108}$, B. De La Cruz ${ }^{108}$, C. Diez Pardos ${ }^{108}$, C. Fernandez Bedoya ${ }^{108}$, J.P. Fern ández Ramos ${ }^{108}$, A. Ferrando ${ }^{108}$, J. Flix ${ }^{108}$, M.C. Fouz ${ }^{108}$, P. Garcia-Abia ${ }^{108}$, O. Gonzalez Lopez ${ }^{108}$, S. Goy Lopez ${ }^{108}$,

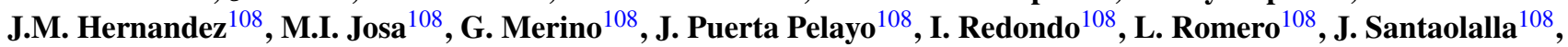
C. Willmott ${ }^{108}$, C. Albajar ${ }^{109}$, J.F. de Trocóniz ${ }^{109}$, J. Cuevas ${ }^{110}$, J. Fernandez Menendez ${ }^{110}$, I. Gonzalez Caballero ${ }^{110}$, L. Lloret Iglesias ${ }^{110}$, J.M. Vizan Garcia ${ }^{110}$, I.J. Cabrillo ${ }^{111}$, A. Calderon ${ }^{111}$, S.H. Chuang ${ }^{111}$, I. Diaz Merino ${ }^{111}$, C. Diez Gonzalez ${ }^{111}$, J. Duarte Campderros ${ }^{111}$, M. Fernandez ${ }^{111}$, G. Gomez ${ }^{111}$, J. Gonzalez Sanchez ${ }^{111}$, R. Gonzalez Suarez ${ }^{111}$, C. Jorda ${ }^{111}$, P. Lobelle Pardo ${ }^{111}$, A. Lopez Virto ${ }^{111}$, J. Marco ${ }^{111}$, R. Marco ${ }^{111}$, C. Martinez Rivero ${ }^{111}$, P. Martinez Ruiz del Arbol ${ }^{111}$, F. Matorras ${ }^{111}$, T. Rodrigo ${ }^{111}$, A. Ruiz Jimeno ${ }^{111}$,

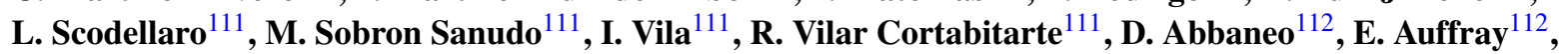
P. Baillon ${ }^{112}$, A.H. Ball ${ }^{112}$, D. Barney ${ }^{112}$, F. Beaudette ${ }^{12, \mathrm{~d}}$, A.J. Bell ${ }^{12, \mathrm{r}}$, D. Benedetti ${ }^{112}$, C. Bernet ${ }^{112, \mathrm{~d}}$, W. Bialas ${ }^{112}$, P. Bloch $^{112}$, A. Bocci ${ }^{112}$, S. Bolognesi ${ }^{112}$, H. Breuker ${ }^{112}$, G. Brona ${ }^{112}$, K. Bunkowski ${ }^{112}$, T. Camporesi ${ }^{112}$, E. Cano ${ }^{112}$, A. Cattai ${ }^{112}$, G. Cerminara ${ }^{112}$, T. Christiansen ${ }^{112}$, J.A. Coarasa Perez ${ }^{112}$, R. Covarelli ${ }^{112}$, B. Curé ${ }^{112}$, T. Dahms ${ }^{112}$, A. De Roeck ${ }^{112}$, A. Elliott-Peisert ${ }^{112}$, W. Funk ${ }^{112}$, A. Gaddi ${ }^{112}$, S. Gennai ${ }^{112}$, H. Gerwig ${ }^{112}$, D. Gigi ${ }^{112}$, K. Gill ${ }^{112}$, D. Giordano ${ }^{112}$, F. Glege ${ }^{112}$, R. Gomez-Reino Garrido ${ }^{112}$, S. Gowdy ${ }^{112}$, L. Guiducci ${ }^{112}$, M. Gouzevitch ${ }^{112}$,

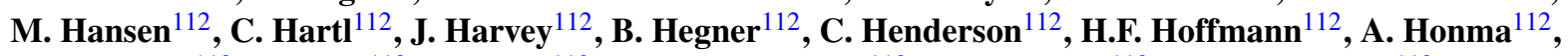

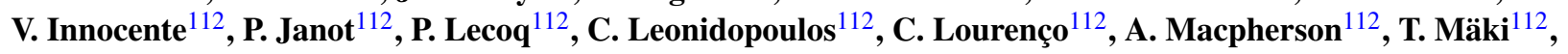


L. Malgeri ${ }^{112}$, M. Mannelli ${ }^{112}$, L. Masetti ${ }^{112}$, F. Meijers ${ }^{112}$, S. Mersi ${ }^{112}$, E. Meschi ${ }^{112}$, R. Moser ${ }^{112}$, M.U. Mozer ${ }^{112}$,

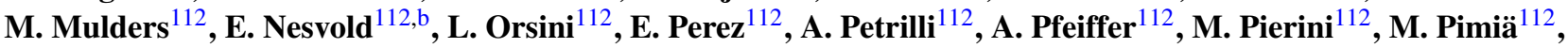

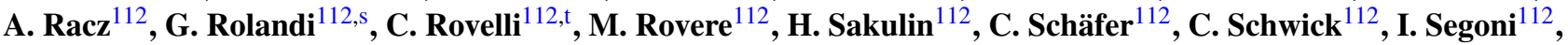
A. Sharma ${ }^{112}$, P. Siegrist ${ }^{112}$, M. Simon ${ }^{112}$, P. Sphicas ${ }^{112, u}$, D. Spiga ${ }^{112}$, M. Spiropulu ${ }^{112, p}$, F. Stöckli ${ }^{112}$, M. Stoye ${ }^{112}$, P. Tropea ${ }^{112}$, A. Tsirou ${ }^{112}$, G.I. Veres ${ }^{112, j}$, P. Vichoudis ${ }^{112}$, M. Voutilainen ${ }^{112}$, W.D. Zeuner ${ }^{112}$, W. Bertl ${ }^{113}$, K. Deiters ${ }^{113}$, W. Erdmann ${ }^{113}$, K. Gabathuler ${ }^{113}$, R. Horisberger ${ }^{113}$, Q. Ingram ${ }^{113}$, H.C. Kaestli ${ }^{113}$, S. König ${ }^{113}$, D. Kotlinski ${ }^{113}$, U. Langenegger ${ }^{113}$, F. Meier ${ }^{113}$, D. Renker ${ }^{113}$, T. Rohe ${ }^{113}$, J. Sibille ${ }^{113, v}$, A. Starodumov ${ }^{113, w}$, L. Caminada ${ }^{114, \mathrm{x}}$, Z. Chen ${ }^{114}$, S. Cittolin ${ }^{114}$, G. Dissertori ${ }^{114}$, M. Dittmar ${ }^{114}$, J. Eugster $^{114}$, K. Freudenreich ${ }^{114}$, C. Grab ${ }^{114}$, A. Hervé ${ }^{114}$, W. Hintz ${ }^{114}$, P. Lecomte ${ }^{114}$, W. Lustermann ${ }^{114}$, C. Marchica ${ }^{114, x}$, P. Meridiani ${ }^{114}$, P. Milenovic ${ }^{114, y}$, F. Moortgat ${ }^{114}$, A. Nardulli ${ }^{114}$, P. Nef ${ }^{114}$, F. Nessi-Tedaldi ${ }^{114}$, L. Pape ${ }^{114}$, F. Pauss ${ }^{114}$, T. Punz ${ }^{114}$, A. Rizzi ${ }^{114}$, F.J. Ronga ${ }^{114}$, L. Sala ${ }^{114}$, A.K. Sanchez ${ }^{114}$, M.-C. Sawley ${ }^{114}$, D. Schinzel ${ }^{114}$, B. Stieger ${ }^{114}$,

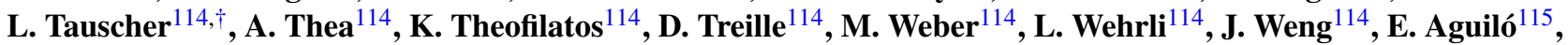
C. Amsler ${ }^{115}$, V. Chiochia ${ }^{115}$, S. De Visscher ${ }^{115}$, C. Favaro ${ }^{115}$, M. Ivova Rikova ${ }^{115}$, A. Jaeger ${ }^{115}$, B. Millan Mejias ${ }^{115}$, C. Regenfus ${ }^{115}$, P. Robmann ${ }^{115}$, T. Rommerskirchen ${ }^{115}$, A. Schmidt ${ }^{115}$, D. Tsirigkas ${ }^{115}$, L. Wilke ${ }^{115}$, Y.H. Chang ${ }^{116}$, K.H. Chen ${ }^{116}$, W.T. Chen ${ }^{116}$, A. Go ${ }^{116}$, C.M. Kuo ${ }^{116}$, S.W. Li ${ }^{116}$, W. Lin ${ }^{116}$, M.H. Liu ${ }^{116}$, Y.J. Lu ${ }^{116}$, J.H. Wu ${ }^{116}$, S.S. Yu ${ }^{116}$, P. Bartalini ${ }^{117}$, P. Chang ${ }^{117}$, Y.H. Chang ${ }^{117}$, Y.W. Chang ${ }^{117}$, Y. Chao ${ }^{117}$, K.F. Chen ${ }^{117}$, W.-S. Hou ${ }^{117}$, Y. Hsiung ${ }^{117}$, K.Y. Kao ${ }^{117}$, Y.J. Lei ${ }^{117}$, S.W. Lin ${ }^{117}$, R.-S. Lu ${ }^{117}$, J.G. Shiu ${ }^{117}$, Y.M. Tzeng ${ }^{117}$, K. Ueno ${ }^{117}$, C.C. Wang ${ }^{117}$, M. Wang ${ }^{117}$, J.T. Wei ${ }^{117}$, A. Adiguzel $^{118}$, A. Ayhan ${ }^{118}$, M.N. Bakirci ${ }^{118}$, S. Cerci ${ }^{118 \text {,aa }}$, Z. Demir ${ }^{118}$, C. Dozen ${ }^{118}$, I. Dumanoglu ${ }^{118}$, E. Eskut ${ }^{118}$, S. Girgis ${ }^{118}$, G. Gökbulut ${ }^{118}$, Y. Güler ${ }^{118}$, E. Gurpinar ${ }^{118}$, I. Hos ${ }^{118}$, E.E. Kangal ${ }^{118}$, T. Karaman ${ }^{118}$, A. Kayis Topaksu ${ }^{118}$, A. Nart ${ }^{118}$, G. Önengüit ${ }^{118}$, K. Ozdemir ${ }^{118}$, S. Ozturk ${ }^{118}$,

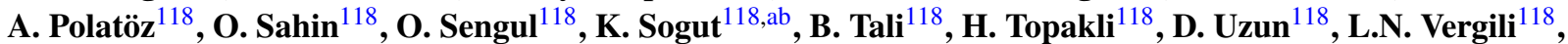
M. Vergili ${ }^{118}$, C. Zorbilmez ${ }^{118}$, I.V. Akin ${ }^{119}$, T. Aliev ${ }^{119}$, S. Bilmis ${ }^{119}$, M. Deniz ${ }^{119}$, H. Gamsizkan ${ }^{119}$, A.M. Guler ${ }^{119}$, K. Ocalan ${ }^{119}$, A. Ozpineci ${ }^{119}$, M. Serin ${ }^{119}$, R. Sever ${ }^{119}$, U.E. Surat ${ }^{119}$, E. Yildirim ${ }^{119}$, M. Zeyrek ${ }^{119}$,

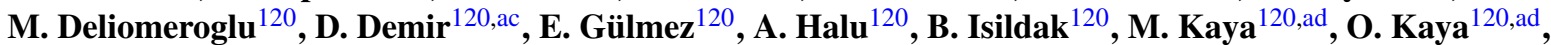

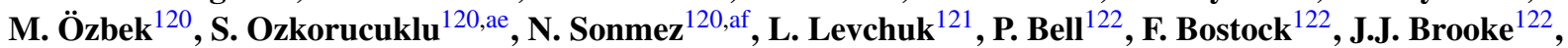
T.L. Cheng ${ }^{122}$, D. Cussans ${ }^{122}$, R. Frazier ${ }^{122}$, J. Goldstein ${ }^{122}$, M. Hansen ${ }^{122}$, G.P. Heath ${ }^{122}$, H.F. Heath ${ }^{122}$, C. Hill ${ }^{122}$,

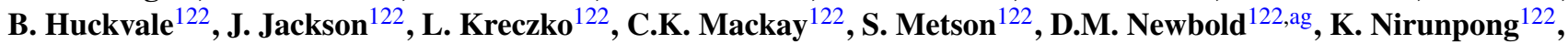
V.J. Smith ${ }^{122}$, S. Ward ${ }^{122}$, L. Basso ${ }^{123}$, K.W. Bell ${ }^{123}$, A. Belyaev ${ }^{123}$, C. Brew ${ }^{123}$, R.M. Brown ${ }^{123}$, B. Camanzi ${ }^{123}$, D.J.A. Cockerill ${ }^{123}$, J.A. Coughlan ${ }^{123}$, K. Harder ${ }^{123}$, S. Harper ${ }^{123}$, B.W. Kennedy ${ }^{123}$, E. Olaiya ${ }^{123}$, D. Petyt ${ }^{123}$, B.C. Radburn-Smith ${ }^{123}$, C.H. Shepherd-Themistocleous ${ }^{123}$, I.R. Tomalin ${ }^{123}$, W.J. Womersley ${ }^{123}$, S.D. Worm ${ }^{123}$, R. Bainbridge ${ }^{124}$, G. Ball ${ }^{124}$, J. Ballin ${ }^{124}$, R. Beuselinck ${ }^{124}$, O. Buchmuller ${ }^{124}$, D. Colling ${ }^{124}$, N. Cripps ${ }^{124}$, M. Cutajar ${ }^{124}$, G. Davies ${ }^{124}$, M. Della Negra ${ }^{124}$, C. Foudas ${ }^{124}$, J. Fulcher ${ }^{124}$, D. Futyan ${ }^{124}$, A. Guneratne Bryer ${ }^{124}$, G. Hall ${ }^{124}$, Z. HatherelI ${ }^{124}$, J. Hays ${ }^{124}$, G. Iles ${ }^{124}$, G. Karapostoli ${ }^{124}$, L. Lyons ${ }^{124}$, A.-M. Magnan ${ }^{124}$, J. Marrouche ${ }^{124}$, R. Nandi ${ }^{124}$, J. Nash ${ }^{124}$, A. Nikitenko ${ }^{124, w}$, A. Papageorgiou ${ }^{124}$, M. Pesaresi ${ }^{124}$, K. Petridis ${ }^{124}$, M. Pioppi ${ }^{124, \text { ah }}$, D.M. Raymond ${ }^{124}$, N. Rompotis ${ }^{124}$, A. Rose $^{124}$, M.J. Ryan ${ }^{124}$, C. Seez ${ }^{124}$, P. Sharp ${ }^{124}$, A. Sparrow ${ }^{124}$, A. Tapper ${ }^{124}$, S. Tourneur ${ }^{124}$, M. Vazquez Acosta ${ }^{124}$, T. Virdee ${ }^{124, b}$, S. Wakefield ${ }^{124}$, D. Wardrope ${ }^{124}$, T. Whyntie ${ }^{124}$, M. Barrett ${ }^{125}$,

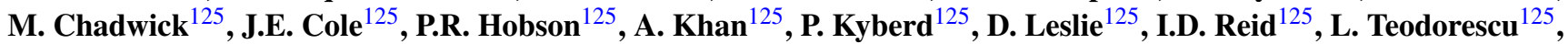
T. Bose ${ }^{126}$, E. Carrera Jarrin ${ }^{126}$, A. Clough ${ }^{126}$, C. Fantasia ${ }^{126}$, A. Heister ${ }^{126}$, J. St. John ${ }^{126}$, P. Lawson ${ }^{126}$,

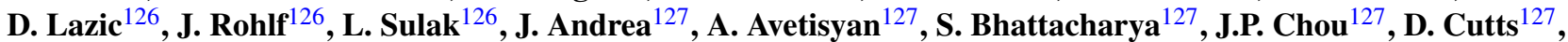
S. Esen $^{127}$, A. Ferapontov ${ }^{127}$, U. Heintz ${ }^{127}$, S. Jabeen ${ }^{127}$, G. Kukartsev ${ }^{127}$, G. Landsberg ${ }^{127}$, M. Narain ${ }^{127}$, D. Nguyen $^{127}$, T. Speer ${ }^{127}$, K.V. Tsang ${ }^{127}$, M.A. Borgia ${ }^{128}$, R. Breedon ${ }^{128}$, M. Calderon De La Barca Sanchez ${ }^{128}$, D. Cebra ${ }^{128}$, M. Chertok ${ }^{128}$, J. Conway ${ }^{128}$, P.T. Cox ${ }^{128}$, J. Dolen ${ }^{128}$, R. Erbacher $^{128}$, E. Friis ${ }^{128}$, W. Ko ${ }^{128}$, A. Kopecky ${ }^{128}$, R. Lander ${ }^{128}$, H. Liu ${ }^{128}$, S. Maruyama ${ }^{128}$, T. Miceli ${ }^{128}$, M. Nikolic ${ }^{128}$, D. Pellett ${ }^{128}$, J. Robles ${ }^{128}$, T. Schwarz ${ }^{128}$, M. Searle ${ }^{128}$, J. Smith ${ }^{128}$, M. Squires ${ }^{128}$, M. Tripathi ${ }^{128}$, R. Vasquez Sierra ${ }^{128}$, C. Veelken ${ }^{128}$, V. Andreev ${ }^{129}$, K. Arisaka ${ }^{129}$, D. Cline ${ }^{129}$, R. Cousins ${ }^{129}$, A. Deisher ${ }^{129}$, S. Erhan ${ }^{129, b}$, C. Farrell ${ }^{129}$, M. Felcini ${ }^{129}$, J. Hauser ${ }^{129}$, M. Ignatenko ${ }^{129}$, C. Jarvis ${ }^{129}$, C. Plager $^{129}$, G. Rakness ${ }^{129}$, P. Schlein ${ }^{129, \dagger}$, J. Tucker ${ }^{129}$, V. Valuev ${ }^{129}$, R. Wallny ${ }^{129}$, J. Babb ${ }^{130}$, R. Clare ${ }^{130}$, J. Ellison ${ }^{130}$, J.W. Gary ${ }^{130}$, F. Giordano ${ }^{130}$, G. Hanson ${ }^{130}$, G.Y. Jeng ${ }^{130}$,

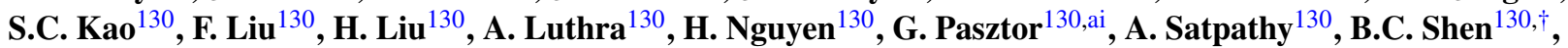
R. Stringer ${ }^{130}$, J. Sturdy ${ }^{130}$, S. Sumowidagdo ${ }^{130}$, R. Wilken ${ }^{130}$, S. Wimpenny ${ }^{130}$, W. Andrews ${ }^{131}$, J.G. Branson ${ }^{131}$, E. Dusinberre ${ }^{131}$, D. Evans ${ }^{131}$, F. Golf ${ }^{131}$, A. Holzner ${ }^{131}$, R. Kelley ${ }^{131}$, M. Lebourgeois ${ }^{131}$, J. Letts ${ }^{131}$, B. Mangano ${ }^{131}$, J. Muelmenstaedt ${ }^{131}$, S. Padhi ${ }^{131}$, C. Palmer ${ }^{131}$, G. Petrucciani ${ }^{131}$, H. Pi ${ }^{131}$, M. Pieri ${ }^{131}$, R. Ranieri ${ }^{131}$, M. Sani ${ }^{131}$,

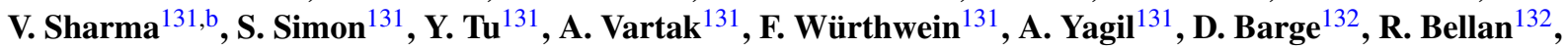


M. Blume ${ }^{132}$, C. Campagnari ${ }^{132}$, M. D’Alfonso ${ }^{132}$, T. Danielson ${ }^{132}$, J. Garberson ${ }^{132}$, J. Incandela ${ }^{132}$, C. Justus ${ }^{132}$, P. Kalavase ${ }^{132}$, S.A. Koay ${ }^{132}$, D. Kovalskyi ${ }^{132}$, V. Krutelyov ${ }^{132}$, J. Lamb ${ }^{132}$, S. Lowette $^{132}$, V. Pavlunin ${ }^{132}$,

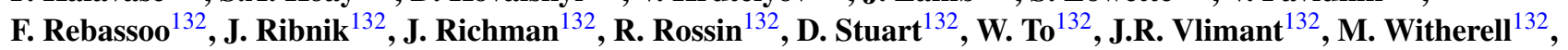

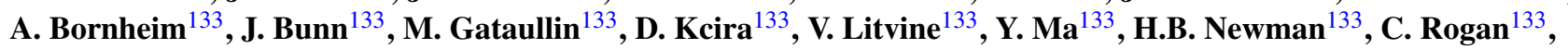
K. Shin ${ }^{133}$, V. Timciuc ${ }^{133}$, P. Traczyk ${ }^{133}$, J. Veverka ${ }^{133}$, R. Wilkinson ${ }^{133}$, Y. Yang ${ }^{133}$, R.Y. Zhu ${ }^{133}$, B. Akgun ${ }^{134}$, R. Carroll ${ }^{134}$, T. Ferguson ${ }^{134}$, D.W. Jang ${ }^{134}$, S.Y. Jun ${ }^{134}$, Y.F. Liu ${ }^{134}$, M. Paulini ${ }^{134}$, J. Russ ${ }^{134}$, N. Terentyev ${ }^{134}$, H. Vogel ${ }^{134}$, I. Vorobiev ${ }^{134}$, J.P. Cumalat ${ }^{135}$, M.E. Dinardo ${ }^{135}$, B.R. Drell ${ }^{135}$, C.J. Edelmaier $^{135}$, W.T. Ford ${ }^{135}$, B. Heyburn ${ }^{135}$, E. Luiggi Lopez ${ }^{135}$, U. Nauenberg ${ }^{135}$, J.G. Smith ${ }^{135}$, K. Stenson ${ }^{135}$, K.A. Ulmer ${ }^{135}$, S.R. Wagner ${ }^{135}$,

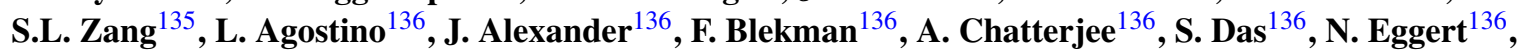
L.J. Fields $^{136}$, L.K. Gibbons ${ }^{136}$, B. Heltsley ${ }^{136}$, K. Henriksson ${ }^{136}$, W. Hopkins ${ }^{136}$, A. Khukhunaishvili ${ }^{136}$, B. Kreis ${ }^{136}$, V. Kuznetsov ${ }^{136}$, G. Nicolas Kaufman ${ }^{136}$, J.R. Patterson ${ }^{136}$, D. Puigh ${ }^{136}$, D. Riley ${ }^{136}$, A. Ryd ${ }^{136}$, M. Saelim ${ }^{136}$, X. Shi ${ }^{136}$, W. Sun ${ }^{136}$, W.D. Teo ${ }^{136}$, J. Thom ${ }^{136}$, J. Thompson ${ }^{136}$, J. Vaughan ${ }^{136}$, Y. Weng ${ }^{136}$, P. Wittich ${ }^{136}$, A. Biselli ${ }^{137}$, G. Cirino ${ }^{137}$, D. Winn ${ }^{137}$, S. Abdullin ${ }^{138}$, M. Albrow ${ }^{138}$, J. Anderson ${ }^{138}$, G. Apollinari ${ }^{138}$, M. Atac ${ }^{138}$,

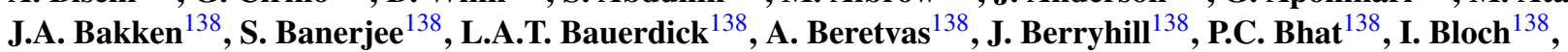
F. Borcherding ${ }^{138}$, K. Burkett ${ }^{138}$, J.N. Butler ${ }^{138}$, V. Chetluru ${ }^{138}$, H.W.K. Cheung ${ }^{138}$, F. Chlebana ${ }^{138}$, S. Cihangir $^{138}$, M. Demarteau ${ }^{138}$, D.P. Eartly ${ }^{138}$, V.D. Elvira ${ }^{138}$, I. Fisk ${ }^{138}$, J. Freeman ${ }^{138}$, Y. Gao ${ }^{138}$, E. Gottschalk ${ }^{138}$, D. Green ${ }^{138}$, O. Gutsche ${ }^{138}$, A. Hahn ${ }^{138}$, J. Hanlon ${ }^{138}$, R.M. Harris ${ }^{138}$, J. Hirschauer ${ }^{138}$, E. James ${ }^{138}$, H. Jensen ${ }^{138}$, M. Johnson ${ }^{138}$, U. Joshi ${ }^{138}$, R. Khatiwada ${ }^{138}$, B. Kilminster ${ }^{138}$, B. Klima ${ }^{138}$, K. Kousouris ${ }^{138}$, S. Kunori ${ }^{138}$, S. Kwan ${ }^{138}$, P. Limon ${ }^{138}$, R. Lipton ${ }^{138}$, J. Lykken ${ }^{138}$, K. Maeshima ${ }^{138}$, J.M. Marraffino ${ }^{138}$, D. Mason ${ }^{138}$, P. McBride ${ }^{138}$, T. McCauley ${ }^{138}$, T. Miao ${ }^{138}$, K. Mishra ${ }^{138}$, S. Mrenna ${ }^{138}$, Y. Musienko ${ }^{138, a j}$, C. Newman-Holmes $^{138}$, V. O'Dell ${ }^{138}$, S. Popescu ${ }^{138}$, R. Pordes ${ }^{138}$, O. Prokofyev ${ }^{138}$, N. Saoulidou ${ }^{138}$, E. Sexton-Kennedy ${ }^{138}$, S. Sharma $^{138}$, R.P. Smith ${ }^{138, \dagger}$, A. Soha ${ }^{138}$, W.J. Spalding ${ }^{138}$, L. Spiegel ${ }^{138}$, P. Tan ${ }^{138}$, L. Taylor ${ }^{138}$, S. Tkaczyk ${ }^{138}$, L. Uplegger ${ }^{138}$, E.W. Vaandering ${ }^{138}$, R. Vidal ${ }^{138}$, J. Whitmore ${ }^{138}$, W. Wu ${ }^{138}$, F. Yumiceva ${ }^{138}$, J.C. Yun ${ }^{138}$, D. Acosta ${ }^{139}$, P. Avery $^{139}$, D. Bourilkov ${ }^{139}$, M. Chen ${ }^{139}$, G.P. Di Giovanni ${ }^{139}$, D. Dobur ${ }^{139}$, A. Drozdetskiy ${ }^{139}$, R.D. Field ${ }^{139}$, M. Fisher ${ }^{139}$, Y. Fu' ${ }^{139}$, I.K. Furic ${ }^{139}$, J. Gartner ${ }^{139}$, B. Kim ${ }^{139}$, S. Klimenko ${ }^{139}$, J. Konigsberg ${ }^{139}$, A. Korytov ${ }^{139}$, K. Kotov ${ }^{139}$, A. Kropivnitskaya ${ }^{139}$, T. Kypreos ${ }^{139}$, K. Matchev ${ }^{139}$, G. Mitselmakher ${ }^{139}$, L. Muniz ${ }^{139}$, Y. Pakhotin ${ }^{139}$,

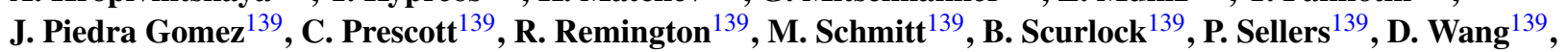
J. Yelton ${ }^{139}$, M. Zakaria ${ }^{139}$, C. Ceron ${ }^{140}$, V. Gaultney ${ }^{140}$, L. Kramer ${ }^{140}$, L.M. Lebolo ${ }^{140}$, S. Linn ${ }^{140}$, P. Markowitz ${ }^{140}$, G. Martinez ${ }^{140}$, D. Mesa ${ }^{140}$, J.L. Rodriguez ${ }^{140}$, T. Adams ${ }^{141}$, A. Askew ${ }^{141}$, J. Chen ${ }^{141}$, B. Diamond ${ }^{141}$, S.V. Gleyzer ${ }^{141}$, J. Haas ${ }^{141}$, S. Hagopian ${ }^{141}$, V. Hagopian ${ }^{141}$, M. Jenkins ${ }^{141}$, K.F. Johnson ${ }^{141}$, H. Prosper ${ }^{141}$, S. Sekmen ${ }^{141}$, V. Veeraraghavan ${ }^{141}$, M.M. Baarmand ${ }^{142}$, S. Guragain ${ }^{142}$, M. Hohlmann ${ }^{142}$, H. Kalakhety ${ }^{142}$, H. Mermerkaya ${ }^{142}$, R. Ralich $^{142}$, I. Vodopiyanov ${ }^{142}$, M.R. Adams ${ }^{143}$, I.M. Anghel ${ }^{143}$, L. Apanasevich ${ }^{143}$, V.E. Bazterra ${ }^{143}$, R.R. Betts ${ }^{143}$, J. Callner ${ }^{143}$, R. Cavanaugh ${ }^{143}$, C. Dragoiu ${ }^{143}$, E.J. Garcia-Solis ${ }^{143}$, C.E. Gerber ${ }^{143}$, D.J. Hofman ${ }^{143}$, S. Khalatian ${ }^{143}$, F. Lacroix ${ }^{143}$, E. Shabalina ${ }^{143}$, A. Smoron ${ }^{143}$, D. Strom ${ }^{143}$, N. Varelas $^{143}$, U. Akgun $^{144}$, E.A. Albayrak ${ }^{144}$, B. Bilki ${ }^{144}$, K. Cankocak ${ }^{144, a k}$, W. Clarida ${ }^{144}$, F. Duru ${ }^{144}$, C.K. Lae $^{144}$, E. McCliment ${ }^{144}$, J.-P. Merlo ${ }^{144}$, A. Mestvirishvili ${ }^{144}$, A. Moeller ${ }^{144}$, J. Nachtman ${ }^{144}$, C.R. Newsom ${ }^{144}$, E. Norbeck $^{144}$, J. Olson ${ }^{144}$, Y. Onel ${ }^{144}$, F. Ozok ${ }^{144}$, S. Sen ${ }^{144}$, J. Wetzel ${ }^{144}$, T. Yetkin ${ }^{144}$, K. Yi ${ }^{144}$, B.A. Barnett ${ }^{145}$, B. Blumenfeld $^{145}$, A. Bonato ${ }^{145}$, C. Eskew ${ }^{145}$, D. Fehling ${ }^{145}$, G. Giurgiu ${ }^{145}$, A.V. Gritsan ${ }^{145}$, Z.J. Guo ${ }^{145}$, G. Hu ${ }^{145}$,

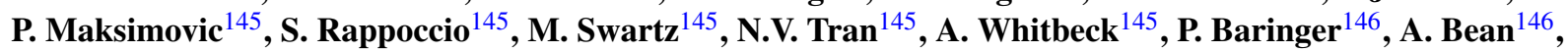

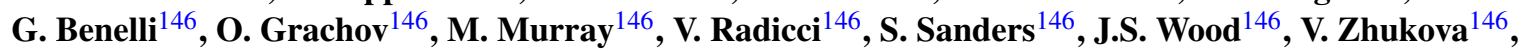
D. Bandurin $^{147}$, T. Bolton ${ }^{147}$, I. Chakaberia ${ }^{147}$, A. Ivanov ${ }^{147}$, K. Kaadze ${ }^{147}$, Y. Maravin ${ }^{147}$, S. Shrestha ${ }^{147}$, I. Svintradze ${ }^{147}$, Z. Wan ${ }^{147}$, J. Gronberg ${ }^{148}$, D. Lange ${ }^{148}$, D. Wright ${ }^{148}$, D. Baden ${ }^{149}$, M. Boutemeur ${ }^{149}$, S.C. Eno ${ }^{149}$, D. Ferencek $^{149}$, N.J. Hadley ${ }^{149}$, R.G. Kellogg ${ }^{149}$, M. Kirn ${ }^{149}$, Y. Lu ${ }^{149}$, A.C. Mignerey ${ }^{149}$, K. Rossato ${ }^{149}$, P. Rumerio $^{149}$, F. Santanastasio ${ }^{149}$, A. Skuja ${ }^{149}$, J. Temple ${ }^{149}$, M.B. Tonjes ${ }^{149}$, S.C. Tonwar ${ }^{149}$, E. Twedt ${ }^{149}$,

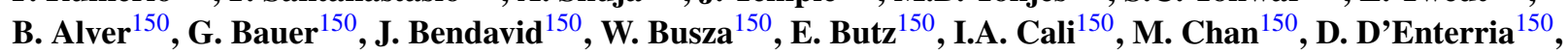
P. Everaerts ${ }^{150}$, G. Gomez Ceballos ${ }^{150}$, M. Goncharov ${ }^{150}$, K.A. Hahn ${ }^{150}$, P. Harris ${ }^{150}$, Y. Kim ${ }^{150}$, M. Klute ${ }^{150}$, Y.-J. Lee ${ }^{150}$, W. Li ${ }^{150}$, C. Loizides ${ }^{150}$, P.D. Luckey ${ }^{150}$, T. Ma ${ }^{150}$, S. Nahn ${ }^{150}$, C. Paus ${ }^{150}$, C. Roland ${ }^{150}$, G. Roland ${ }^{150}$, M. Rudolph ${ }^{150}$, G.S.F. Stephans ${ }^{150}$, K. Sumorok ${ }^{150}$, K. Sung ${ }^{150}$, E.A. Wenger ${ }^{150}$, B. Wyslouch ${ }^{150}$, S. Xie ${ }^{150}$, M. Yang ${ }^{150}$, Y. Yilmaz ${ }^{150}$, A.S. Yoon ${ }^{150}$, M. Zanetti ${ }^{150}$, P. Cole ${ }^{151}$, S.I. Cooper ${ }^{151}$, P. Cushman ${ }^{151}$, B. Dahmes ${ }^{151}$, A. De Benedetti ${ }^{151}$, P.R. Dudero ${ }^{151}$, G. Franzoni ${ }^{151}$, J. Haupt ${ }^{151}$, K. Klapoetke ${ }^{151}$, Y. Kubota ${ }^{151}$, J. Mans ${ }^{151}$, V. Rekovic $^{151}$, R. Rusack ${ }^{151}$, M. Sasseville ${ }^{151}$, A. Singovsky ${ }^{151}$, L.M. Cremaldi ${ }^{152}$, R. Godang ${ }^{152}$, R. Kroeger ${ }^{152}$,

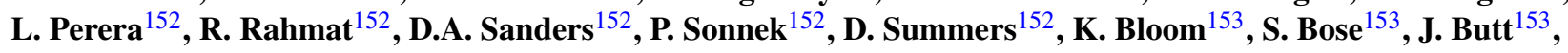


D.R. Claes ${ }^{153}$, A. Dominguez ${ }^{153}$, M. Eads ${ }^{153}$, J. Keller ${ }^{153}$, T. Kelly ${ }^{153}$, I. Kravchenko ${ }^{153}$, J. Lazo-Flores ${ }^{153}$, C. Lundstedt ${ }^{153}$, H. Malbouisson ${ }^{153}$, S. Malik ${ }^{153}$, G.R. Snow ${ }^{153}$, U. Baur ${ }^{154}$, I. Iashvili ${ }^{154}$, A. Kharchilava ${ }^{154}$, A. Kumar ${ }^{154}$, K. Smith ${ }^{154}$, J. Zennamo ${ }^{154}$, G. Alverson ${ }^{155}$, E. Barberis ${ }^{155}$, D. Baumgartel ${ }^{155}$, O. Boeriu ${ }^{155}$, M. Chasco ${ }^{155}$, S. Reucroft ${ }^{155}$, J. Swain ${ }^{155}$, D. Wood ${ }^{155}$, J. Zhang ${ }^{155}$, A. Anastassov ${ }^{156}$, A. Kubik ${ }^{156}$,

R.A. Ofierzynski ${ }^{156}$, A. Pozdnyakov ${ }^{156}$, M. Schmitt ${ }^{156}$, S. Stoynev $^{156}$, M. Velasco ${ }^{156}$, S. Won $^{156}$, L. Antonelli ${ }^{157}$, D. Berry ${ }^{157}$, M. Hildreth ${ }^{157}$, C. Jessop ${ }^{157}$, D.J. Karmgard ${ }^{157}$, J. Kolb ${ }^{157}$, T. Kolberg ${ }^{157}$, K. Lannon ${ }^{157}$, S. Lynch ${ }^{157}$, N. Marinelli ${ }^{157}$, D.M. Morse ${ }^{157}$, R. Ruchti ${ }^{157}$, J. Slaunwhite ${ }^{157}$, N. Valls ${ }^{157}$, J. Warchol ${ }^{157}$, M. Wayne ${ }^{157}$, J. Ziegler ${ }^{157}$, B. Bylsma ${ }^{158}$, L.S. Durkin ${ }^{158}$, J. Gu ${ }^{158}$, P. Killewald ${ }^{158}$, T.Y. Ling ${ }^{158}$, M. Rodenburg ${ }^{158}$, G. Williams ${ }^{158}$, N. Adam ${ }^{159}$, E. Berry ${ }^{159}$, P. Elmer ${ }^{159}$, D. Gerbaudo ${ }^{159}$, V. Halyo ${ }^{159}$, A. Hunt ${ }^{159}$, J. Jones ${ }^{159}$, E. Laird ${ }^{159}$, D. Lopes Pegna ${ }^{159}$, D. Marlow ${ }^{159}$, T. Medvedeva ${ }^{159}$, M. Mooney ${ }^{159}$, J. Olsen ${ }^{159}$, P. Pirouéé ${ }^{159}$, D. Stickland ${ }^{159}$, C. Tully ${ }^{159}$, J.S. Werner ${ }^{159}$, A. Zuranski ${ }^{159}$, J.G. Acosta ${ }^{160}$, X.T. Huang ${ }^{160}$, A. Lopez ${ }^{160}$, H. Mendez ${ }^{160}$, S. Oliveros ${ }^{160}$, J.E. Ramirez Vargas ${ }^{160}$, A. Zatzerklyaniy ${ }^{160}$, E. Alagoz ${ }^{161}$, V.E. Barnes ${ }^{161}$, G. Bolla ${ }^{161}$, L. Borrello ${ }^{161}$, D. Bortoletto ${ }^{161}$, A. Everett ${ }^{161}$, A.F. Garfinkel ${ }^{161}$, Z. Gecse ${ }^{161}$, L. Gutay ${ }^{161}$, M. Jones ${ }^{161}$, O. Koybasi ${ }^{161}$,

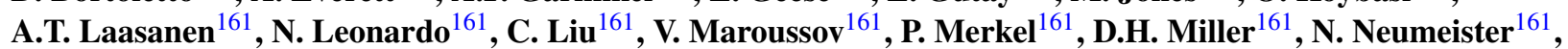
K. Potamianos ${ }^{161}$, I. Shipsey ${ }^{161}$, D. Silvers ${ }^{161}$, H.D. Yoo ${ }^{161}$, J. Zablocki ${ }^{161}$, Y. Zheng ${ }^{161}$, P. Jindal ${ }^{162}$, N. Parashar ${ }^{162}$, V. Cuplov ${ }^{163}$, K.M. Ecklund ${ }^{163}$, F.J.M. Geurts ${ }^{163}$, J.H. Liu ${ }^{163}$, J. Morales ${ }^{163}$, B.P. Padley ${ }^{163}$, R. Redjimi ${ }^{163}$, J. Roberts ${ }^{163}$, B. Betchart ${ }^{164}$, A. Bodek ${ }^{164}$, Y.S. Chung ${ }^{164}$, P. de Barbaro ${ }^{164}$, R. Demina ${ }^{164}$, Y. Eshaq ${ }^{164}$, H. Flacher ${ }^{164}$, A. Garcia-Bellido ${ }^{164}$, P. Goldenzweig ${ }^{164}$, Y. Gotra ${ }^{164}$, J. Han ${ }^{164}$, A. Harel ${ }^{164}$, D.C. Miner ${ }^{164}$, D. Orbaker ${ }^{164}$, G. Petrillo ${ }^{164}$, D. Vishnevskiy ${ }^{164}$, M. Zielinski ${ }^{164}$, A. Bhatti ${ }^{165}$, L. Demortier ${ }^{165}$, K. Goulianos ${ }^{165}$, K. Hatakeyama ${ }^{165}$, G. Lungu ${ }^{165}$, C. Mesropian ${ }^{165}$, M. Yan ${ }^{165}$, O. Atramentov ${ }^{166}$, Y. Gershtein ${ }^{166}$, R. Gray ${ }^{166}$, E. Halkiadakis $^{166}$, D. Hidas ${ }^{166}$, D. Hits ${ }^{166}$, A. Lath ${ }^{166}$, K. Rose ${ }^{166}$, S. Schnetzer ${ }^{166}$, S. Somalwar ${ }^{166}$, R. Stone ${ }^{166}$, S. Thomas ${ }^{166}$, G. Cerizza ${ }^{167}$, M. Hollingsworth ${ }^{167}$, S. Spanier ${ }^{167}$, Z.C. Yang $^{167}$, A. York $^{167}$, J. Asaadi ${ }^{168}$, R. Eusebi ${ }^{168}$, J. Gilmore ${ }^{168}$, A. Gurrola ${ }^{168}$, T. Kamon ${ }^{168}$, V. Khotilovich ${ }^{168}$, R. Montalvo ${ }^{168}$, C.N. Nguyen ${ }^{168}$, J. Pivarski ${ }^{168}$, A. Safonov ${ }^{168}$, S. Sengupta ${ }^{168}$, D. Toback ${ }^{168}$, M. Weinberger ${ }^{168}$, N. Akchurin ${ }^{169}$, C. Bardak ${ }^{169}$, J. Damgov ${ }^{169}$, C. Jeong ${ }^{169}$, K. Kovitanggoon ${ }^{169}$, S.W. Lee ${ }^{169}$, P. Mane ${ }^{169}$, Y. Roh $^{169}$, A. Sill ${ }^{169}$, I. Volobouev ${ }^{169}$, R. Wigmans ${ }^{169}$, E. Yazgan ${ }^{169}$, E. Appelt ${ }^{170}$, E. Brownson ${ }^{170}$, D. Engh $^{170}$, C. Florez ${ }^{170}$, W. Gabella ${ }^{170}$, W. Johns ${ }^{170}$, P. Kurt ${ }^{170}$, C. Maguire ${ }^{170}$, A. Melo ${ }^{170}$, P. Sheldon ${ }^{170}$, J. Velkovska ${ }^{170}$, M.W. Arenton ${ }^{171}$, M. Balazs ${ }^{171}$, S. Boutle ${ }^{171}$, M. Buehler ${ }^{171}$, S. Conetti ${ }^{171}$, B. Cox ${ }^{171}$, R. Hirosky ${ }^{171}$, A. Ledovskoy ${ }^{171}$, C. Neu ${ }^{171}$, R. Yohay ${ }^{171}$, S. Gollapinni ${ }^{172}$, K. Gunthoti ${ }^{172}$, R. Harr ${ }^{172}$, P.E. Karchin ${ }^{172}$, M. Mattson ${ }^{172}$, C. Milstène ${ }^{172}$, A. Sakharov ${ }^{172}$, M. Anderson ${ }^{173}$, M. Bachtis ${ }^{173}$, J.N. Bellinger ${ }^{173}$, D. Carlsmith ${ }^{173}$, S. Dasu ${ }^{173}$, S. Dutta ${ }^{173}$, J. Efron ${ }^{173}$, L. Gray ${ }^{173}$, K.S. Grogg ${ }^{173}$, M. Grothe ${ }^{173}$, M. Herndon ${ }^{173}$, P. Klabbers ${ }^{173}$, J. Klukas ${ }^{173}$, A. Lanaro ${ }^{173}$, C. Lazaridis $^{173}$, J. Leonard ${ }^{173}$,

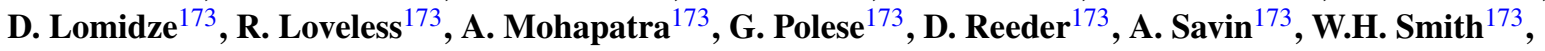
J. Swanson ${ }^{173}$, M. Weinberg ${ }^{173}$

\footnotetext{
${ }^{1}$ Yerevan Physics Institute, Yerevan, Armenia

${ }^{2}$ Institut für Hochenergiephysik der OeAW, Wien, Austria

${ }^{3}$ National Centre for Particle and High Energy Physics, Minsk, Belarus

${ }^{4}$ Universiteit Antwerpen, Antwerpen, Belgium

${ }^{5}$ Vrije Universiteit Brussel, Brussel, Belgium

${ }^{6}$ Université Libre de Bruxelles, Bruxelles, Belgium

${ }^{7}$ Ghent University, Ghent, Belgium

${ }^{8}$ Université Catholique de Louvain, Louvain-la-Neuve, Belgium

${ }^{9}$ Université de Mons, Mons, Belgium

${ }^{10}$ Centro Brasileiro de Pesquisas Fisicas, Rio de Janeiro, Brazil

${ }^{11}$ Universidade do Estado do Rio de Janeiro, Rio de Janeiro, Brazil

${ }^{12}$ Instituto de Fisica Teorica, Universidade Estadual Paulista, Sao Paulo, Brazil

${ }^{13}$ Institute for Nuclear Research and Nuclear Energy, Sofia, Bulgaria

${ }^{14}$ University of Sofia, Sofia, Bulgaria

${ }^{15}$ Institute of High Energy Physics, Beijing, China

${ }^{16}$ State Key Lab. of Nucl. Phys. and Tech., Peking University, Beijing, China

${ }^{17}$ Universidad de Los Andes, Bogota, Colombia

${ }^{18}$ Technical University of Split, Split, Croatia

${ }^{19}$ University of Split, Split, Croatia

${ }^{20}$ Institute Rudjer Boskovic, Zagreb, Croatia

${ }^{21}$ University of Cyprus, Nicosia, Cyprus

${ }^{22}$ Academy of Scientific Research and Technology of the Arab Republic of Egypt, Egyptian Network of High Energy Physics, Cairo, Egypt

${ }^{23}$ National Institute of Chemical Physics and Biophysics, Tallinn, Estonia

${ }^{24}$ Department of Physics, University of Helsinki, Helsinki, Finland
} 
${ }^{25}$ Helsinki Institute of Physics, Helsinki, Finland

${ }^{26}$ Lappeenranta University of Technology, Lappeenranta, Finland

${ }^{27}$ Laboratoire d'Annecy-le-Vieux de Physique des Particules, IN2P3-CNRS, Annecy-le-Vieux, France

${ }^{28}$ DSM/IRFU, CEA/Saclay, Gif-sur-Yvette, France

${ }^{29}$ Laboratoire Leprince-Ringuet, Ecole Polytechnique, IN2P3-CNRS, Palaiseau, France

${ }^{30}$ Institut Pluridisciplinaire Hubert Curien, Université de Strasbourg, Université de Haute Alsace Mulhouse, CNRS/IN2P3, Strasbourg, France

${ }^{31}$ Centre de Calcul de l'Institut National de Physique Nucleaire et de Physique des Particules (IN2P3), Villeurbanne, France

${ }^{32}$ Université de Lyon, Université Claude Bernard Lyon 1, CNRS-IN2P3, Institut de Physique Nucléaire de Lyon, Villeurbanne, France

${ }^{33}$ E. Andronikashvili Institute of Physics, Academy of Science, Tbilisi, Georgia

${ }^{34}$ RWTH Aachen University, I. Physikalisches Institut, Aachen, Germany

${ }^{35}$ RWTH Aachen University, III. Physikalisches Institut A, Aachen, Germany

${ }^{36}$ RWTH Aachen University, III. Physikalisches Institut B, Aachen, Germany

${ }^{37}$ Deutsches Elektronen-Synchrotron, Hamburg, Germany

${ }^{38}$ University of Hamburg, Hamburg, Germany

${ }^{39}$ Institut für Experimentelle Kernphysik, Karlsruhe, Germany

${ }^{40}$ Institute of Nuclear Physics "Demokritos", Aghia Paraskevi, Greece

${ }^{41}$ University of Athens, Athens, Greece

${ }^{42}$ University of Ioánnina, Ioánnina, Greece

${ }^{43}$ KFKI Research Institute for Particle and Nuclear Physics, Budapest, Hungary

${ }^{44}$ Institute of Nuclear Research ATOMKI, Debrecen, Hungary

${ }^{45}$ University of Debrecen, Debrecen, Hungary

${ }^{46}$ Panjab University, Chandigarh, India

${ }^{47}$ University of Delhi, Delhi, India

${ }^{48}$ Bhabha Atomic Research Centre, Mumbai, India

${ }^{49}$ Tata Institute of Fundamental Research-EHEP, Mumbai, India

${ }^{50}$ Tata Institute of Fundamental Research-HECR, Mumbai, India

${ }^{51}$ Institute for Studies in Theoretical Physics \& Mathematics (IPM), Tehran, Iran

${ }^{52}$ INFN Sezione di Bari, Bari, Italy

${ }^{53}$ Università di Bari, Bari, Italy

${ }^{54}$ Politecnico di Bari, Bari, Italy

${ }^{55}$ INFN Sezione di Bologna, Bologna, Italy

${ }^{56}$ Università di Bologna, Bologna, Italy

${ }^{57}$ INFN Sezione di Catania, Catania, Italy

${ }^{58}$ Università di Catania, Catania, Italy

${ }^{59}$ INFN Sezione di Firenze, Firenze, Italy

${ }^{60}$ Università di Firenze, Firenze, Italy

${ }^{61}$ INFN Laboratori Nazionali di Frascati, Frascati, Italy

${ }^{62}$ INFN Sezione di Genova, Genova, Italy

${ }^{63}$ INFN Sezione di Milano-Biccoca, Milano, Italy

${ }^{64}$ Università di Milano-Bicocca, Milano, Italy

${ }^{65}$ INFN Sezione di Napoli, Napoli, Italy

${ }^{66}$ Università di Napoli "Federico II", Napoli, Italy

${ }^{67}$ INFN Sezione di Padova, Padova, Italy

${ }^{68}$ Università di Padova, Padova, Italy

${ }^{69}$ Università di Trento (Trento), Padova, Italy

${ }^{70}$ INFN Sezione di Pavia, Pavia, Italy

${ }^{71}$ Università di Pavia, Pavia, Italy

${ }^{72}$ INFN Sezione di Perugia, Perugia, Italy

${ }^{73}$ Università di Perugia, Perugia, Italy

${ }_{75}^{74}$ INFN Sezione di Pisa, Pisa, Italy

${ }^{75}$ Università di Pisa, Pisa, Italy

${ }^{76}$ Scuola Normale Superiore di Pisa, Pisa, Italy

${ }^{77}$ INFN Sezione di Roma, Roma, Italy

${ }^{78}$ Università di Roma "La Sapienza", Roma, Italy

${ }^{79}$ INFN Sezione di Torino, Torino, Italy

${ }^{80}$ Università di Torino, Torino, Italy

${ }^{81}$ Università del Piemonte Orientale (Novara), Torino, Italy

${ }^{82}$ INFN Sezione di Trieste, Trieste, Italy

${ }^{83}$ Università di Trieste, Trieste, Italy

${ }^{84}$ Chonbuk National University, Chonju, Korea

${ }^{85}$ Kyungpook National University, Daegu, Korea

${ }^{86}$ Chonnam National University, Institute for Universe and Elementary Particles, Kwangju, Korea

${ }^{87}$ Korea University, Seoul, Korea

${ }^{88}$ University of Seoul, Seoul, Korea

${ }^{89}$ Sungkyunkwan University, Suwon, Korea 
${ }^{90}$ Vilnius University, Vilnius, Lithuania

${ }^{91}$ Universidad Iberoamericana, Mexico City, Mexico

${ }^{92}$ Benemerita Universidad Autonoma de Puebla, Puebla, Mexico

${ }^{93}$ Universidad Autónoma de San Luis Potosí, San Luis Potosí, Mexico

${ }^{94}$ University of Auckland, Auckland, New Zealand

${ }^{95}$ University of Canterbury, Christchurch, New Zealand

${ }^{96}$ National Centre for Physics, Quaid-I-Azam University, Islamabad, Pakistan

${ }^{97}$ Institute of Experimental Physics, Warsaw, Poland

${ }^{98}$ Soltan Institute for Nuclear Studies, Warsaw, Poland

${ }^{99}$ Laboratório de Instrumentaç ao e Física Experimental de Partículas, Lisboa, Portugal

${ }^{100}$ Joint Institute for Nuclear Research, Dubna, Russia

${ }^{101}$ Petersburg Nuclear Physics Institute, Gatchina (St Petersburg), Russia

${ }^{102}$ Institute for Nuclear Research, Moscow, Russia

${ }^{103}$ Institute for Theoretical and Experimental Physics, Moscow, Russia

${ }^{104}$ Moscow State University, Moscow, Russia

${ }^{105}$ P.N. Lebedev Physical Institute, Moscow, Russia

${ }^{106}$ State Research Center of Russian Federation, Institute for High Energy Physics, Protvino, Russia

${ }^{107}$ University of Belgrade, Faculty of Physics and Vinca Institute of Nuclear Sciences, Belgrade, Serbia

${ }^{108}$ Centro de Investigaciones Energéticas Medioambientales y Tecnológicas (CIEMAT), Madrid, Spain

${ }^{109}$ Universidad Autónoma de Madrid, Madrid, Spain

${ }^{110}$ Universidad de Oviedo, Oviedo, Spain

${ }^{111}$ Instituto de Física de Cantabria (IFCA), CSIC-Universidad de Cantabria, Santander, Spain

${ }^{112}$ CERN, European Organization for Nuclear Research, Geneva, Switzerland

${ }^{113}$ Paul Scherrer Institut, Villigen, Switzerland

${ }^{114}$ Institute for Particle Physics, ETH Zurich, Zurich, Switzerland

${ }^{115}$ Universität Zürich, Zurich, Switzerland

${ }^{116}$ National Central University, Chung-Li, Taiwan

${ }^{117}$ National Taiwan University (NTU), Taipei, Taiwan

${ }^{118}$ Cukurova University, Adana, Turkey

${ }_{119}^{19}$ Middle East Technical University, Physics Department, Ankara, Turkey

${ }^{120}$ Bogaziçi University, Department of Physics, Istanbul, Turkey

${ }^{121}$ National Scientific Center, Kharkov Institute of Physics and Technology, Kharkov, Ukraine

${ }^{122}$ University of Bristol, Bristol, UK

${ }^{123}$ Rutherford Appleton Laboratory, Didcot, UK

${ }^{124}$ Imperial College, University of London, London, UK

${ }^{125}$ Brunel University, Uxbridge, UK

${ }^{126}$ Boston University, Boston, USA

${ }^{127}$ Brown University, Providence, USA

${ }^{128}$ University of California, Davis, Davis, USA

${ }^{129}$ University of California, Los Angeles, Los Angeles, USA

${ }^{130}$ University of California, Riverside, Riverside, USA

${ }^{131}$ University of California, San Diego, La Jolla, USA

${ }^{132}$ University of California, Santa Barbara, Santa Barbara, USA

${ }^{133}$ California Institute of Technology, Pasadena, USA

${ }^{134}$ Carnegie Mellon University, Pittsburgh, USA

${ }^{135}$ University of Colorado at Boulder, Boulder, USA

${ }^{136}$ Cornell University, Ithaca, USA

${ }^{137}$ Fairfield University, Fairfield, USA

${ }^{138}$ Fermi National Accelerator Laboratory, Batavia, USA

${ }^{139}$ University of Florida, Gainesville, USA

${ }^{140}$ Florida International University, Miami, USA

${ }^{141}$ Florida State University, Tallahassee, USA

${ }^{142}$ Florida Institute of Technology, Melbourne, USA

${ }^{143}$ University of Illinois at Chicago (UIC), Chicago, USA

${ }^{144}$ The University of Iowa, Iowa City, USA

${ }^{145}$ Johns Hopkins University, Baltimore, USA

${ }^{146}$ The University of Kansas, Lawrence, USA

${ }^{147}$ Kansas State University, Manhattan, USA

${ }^{148}$ Lawrence Livermore National Laboratory, Livermore, USA

${ }^{149}$ University of Maryland, College Park, USA

${ }^{150}$ Massachusetts Institute of Technology, Cambridge, USA

${ }^{151}$ University of Minnesota, Minneapolis, USA

${ }^{152}$ University of Mississippi, University, USA

${ }^{153}$ University of Nebraska-Lincoln, Lincoln, USA

${ }^{154}$ State University of New York at Buffalo, Buffalo, USA 
${ }^{155}$ Northeastern University, Boston, USA

${ }^{156}$ Northwestern University, Evanston, USA

${ }^{157}$ University of Notre Dame, Notre Dame, USA

${ }^{158}$ The Ohio State University, Columbus, USA

${ }^{159}$ Princeton University, Princeton, USA

${ }^{160}$ University of Puerto Rico, Mayaguez, USA

${ }^{161}$ Purdue University, West Lafayette, USA

${ }^{162}$ Purdue University Calumet, Hammond, USA

${ }^{163}$ Rice University, Houston, USA

${ }^{164}$ University of Rochester, Rochester, USA

${ }^{165}$ The Rockefeller University, New York, USA

${ }^{166}$ Rutgers, the State University of New Jersey, Piscataway, USA

${ }^{167}$ University of Tennessee, Knoxville, USA

${ }^{168}$ Texas A\&M University, College Station, USA

${ }^{169}$ Texas Tech University, Lubbock, USA

${ }^{170}$ Vanderbilt University, Nashville, USA

${ }^{171}$ University of Virginia, Charlottesville, USA

${ }^{172}$ Wayne State University, Detroit, USA

${ }^{173}$ University of Wisconsin, Madison, USA

Received: 12 July 2010 / Revised: 22 October 2010 / Published online: 24 November 2010

(C) CERN for the benefit of the CMS collaboration 2010. This article is published with open access at Springerlink.com

a e-mail: cms-publication-committee-chair@cern.ch

${ }^{\mathrm{b}}$ Also at CERN, European Organization for Nuclear Research, Geneva, Switzerland.

${ }^{\mathrm{c}}$ Also at Universidade Federal do ABC, Santo Andre, Brazil.

${ }^{\mathrm{d}}$ Also at Laboratoire Leprince-Ringuet, Ecole Polytechnique, IN2P3CNRS, Palaiseau, France.

${ }^{e}$ Also at Fayoum University, El-Fayoum, Egypt.

${ }^{f}$ Also at Soltan Institute for Nuclear Studies, Warsaw, Poland.

${ }^{\mathrm{g}}$ Also at Université de Haute-Alsace, Mulhouse, France.

${ }^{\mathrm{h}}$ Also at Moscow State University, Moscow, Russia.

${ }^{i}$ Also at Institute of Nuclear Research ATOMKI, Debrecen, Hungary.

${ }^{\mathrm{j}}$ Also at Eötvös Loránd University, Budapest, Hungary.

${ }^{\mathrm{k}}$ Also at Tata Institute of Fundamental Research - HECR, Mumbai, India.

${ }^{1}$ Also at University of Visva-Bharati, Santiniketan, India.

mAlso at Facolta' Ingegneria Università di Roma "La Sapienza", Roma, Italy.

${ }^{\mathrm{n}}$ Also at Università della Basilicata, Potenza, Italy.

${ }^{\circ}$ Also at Laboratori Nazionali di Legnaro dell' INFN, Legnaro, Italy.

$\mathrm{p}$ Also at California Institute of Technology, Pasadena, USA.

${ }^{\mathrm{q}}$ Also at Faculty of Physics of University of Belgrade, Belgrade, Serbia.

${ }^{\mathrm{r}}$ Also at Université de Genève, Geneva, Switzerland.

${ }^{\text {s} A l s o ~ a t ~ S c u o l a ~ N o r m a l e ~ e ~ S e z i o n e ~ d e l l ' ~ I N F N, ~ P i s a, ~ I t a l y . ~}$

tAlso at INFN Sezione di Roma; Università di Roma "La Sapienza", Roma, Italy.

${ }^{\mathrm{u}}$ Also at University of Athens, Athens, Greece.

${ }^{v}$ Also at The University of Kansas, Lawrence, USA.

${ }^{\mathrm{w}}$ Also at Institute for Theoretical and Experimental Physics, Moscow, Russia.

${ }^{x}$ Also at Paul Scherrer Institut, Villigen, Switzerland.

${ }^{y}$ Also at University of Belgrade, Faculty of Physics and Vinca Institute of Nuclear Sciences, Belgrade, Serbia.
Abstract The first LHC pp collisions at centre-of-mass energies of 0.9 and $2.36 \mathrm{TeV}$ were recorded by the CMS detector in December 2009. The trajectories of charged particles produced in the collisions were reconstructed using the all-silicon Tracker and their momenta were measured in the $3.8 \mathrm{~T}$ axial magnetic field. Results from the Tracker commissioning are presented including studies of timing, efficiency, signal-to-noise, resolution, and ionization energy. Reconstructed tracks are used to benchmark the performance in terms of track and vertex resolutions, reconstruction of decays, estimation of ionization energy loss, as well as identification of photon conversions, nuclear interactions, and heavy-flavour decays.

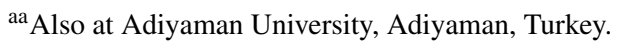

${ }^{\mathrm{ab}}$ Also at Mersin University, Mersin, Turkey.

${ }^{\text {ac }}$ Also at Izmir Institute of Technology, Izmir, Turkey.

${ }^{\text {ad }}$ Also at Kafkas University, Kars, Turkey.

${ }^{a e}$ Also at Suleyman Demirel University, Isparta, Turkey.

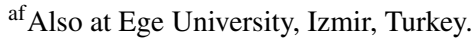

ag Also at Rutherford Appleton Laboratory, Didcot, United Kingdom.

${ }^{\text {ah }}$ Also at INFN Sezione di Perugia; Università di Perugia, Perugia, Italy.

ai Also at KFKI Research Institute for Particle and Nuclear Physics, Budapest, Hungary.

aj Also at Institute for Nuclear Research, Moscow, Russia.

${ }^{\mathrm{ak}}$ Also at Istanbul Technical University, Istanbul, Turkey.

${ }^{\dagger}$ Deceased.
} 


\section{Introduction}

The Compact Muon Solenoid (CMS) [1] is a general purpose detector at the Large Hadron Collider (LHC) of CERN. It has been designed primarily to perform new physics studies at the highest energies achievable with the LHC. The main components of CMS are a muon detection system, electromagnetic and hadronic calorimeters, and an inner tracking system (Tracker). The Tracker provides robust, efficient, and precise reconstruction of the charged particle trajectories inside a $3.8 \mathrm{~T}$ axial magnetic field. The nominal momentum resolution is typically $0.7(5.0) \%$ at $1(1000) \mathrm{GeV} / c$ in the central region and the impact parameter resolution for high-momentum tracks is typically $10 \mu \mathrm{m}$.

The reconstructed tracks of charged particles are among the most fundamental objects in the reconstruction of pp collisions. Tracks are used in the reconstruction of electrons, muons, hadrons, taus, and jets as well as in the determination of the primary interaction vertices. In addition, tracks may be used to identify $b$ jets, in particular through evidence of a displaced vertex associated with a given jet.

This paper describes the performance of the Tracker, which was evaluated with collision data from early LHC operations at centre-of-mass energies of 0.9 and $2.36 \mathrm{TeV}$. The next section contains a brief description of the Tracker. Section 3 illustrates the LHC data and conditions that underlie the analysis. Results obtained from the commissioning of the Pixel and Silicon Strip detectors are described in Sect. 4. Section 5 describes the track reconstruction and Sect. 6 presents tracking results demonstrating the overall performance of the Tracker. In particular, reconstructed tracks are used for track and vertex resolution measurements, the reconstruction of hadron decays, the estimation of ionization energy loss, the identification of photon conversions and nuclear interactions, and $\mathrm{b}$ tagging. Finally, conclusions are presented in Sect. 7.

\section{Tracker description}

The CMS experiment uses a right-handed coordinate system, with the origin at the nominal interaction point, the $x$ axis pointing to the centre of the LHC ring, the $y$ axis pointing up (perpendicular to the LHC plane) and the $z$ axis along the anticlockwise-beam direction. The azimuthal angle $\phi$ is measured in the $x y$ plane, with $\phi=0$ along the positive $x$ axis and $\phi=\pi / 2$ along the positive $y$ axis.

The CMS Tracker [1], shown in Fig. 1, consists of two main detectors: a silicon pixel detector, covering the region from 4 to $15 \mathrm{~cm}$ in radius, and $49 \mathrm{~cm}$ on either side of the collision point along the LHC beam axis, and a silicon strip detector, covering the region from 25 to $110 \mathrm{~cm}$ in radius, and within $280 \mathrm{~cm}$ on either side of the collision point along the LHC beam axis.

The CMS silicon pixel detector has 66 million active elements instrumenting a surface area of about $1 \mathrm{~m}^{2}$. It is designed to provide the determination of three high precision three-dimensional points on track trajectories. The detector consists of three concentric cylindrical barrel layers and four fan-blade disks which close the barrel ends. The barrel layers have an active length of $53 \mathrm{~cm}$ and are located at average radii of $4.3,7.3$, and $10.2 \mathrm{~cm}$. The endcap disks instrument the regions between radii 4.8 and $14.4 \mathrm{~cm}$ at mean longitudinal distances of 35.5 and $48.5 \mathrm{~cm}$ from the interaction point. The system provides efficient three-hit coverage in the region of pseudorapidity $|\eta|<2.2$ and efficient twohit coverage in the region $|\eta|<2.5$. The active elements are n-in-n $100 \mu \mathrm{m} \times 150 \mu \mathrm{m}$ pixels [1] which are oriented with the smaller pitch in the azimuthal direction in the barrel and the radial direction in the disks. The 3.8 T magnetic field in CMS causes significant azimuthal Lorentz drift of the collected electrons in the pixel barrel which enhances the azimuthal charge sharing and therefore improves the resolution in that direction. The blades of the endcap disks are rotated by 20 degrees about their radial axes with respect to
Fig. $1 r-z$ slice of the CMS Tracker

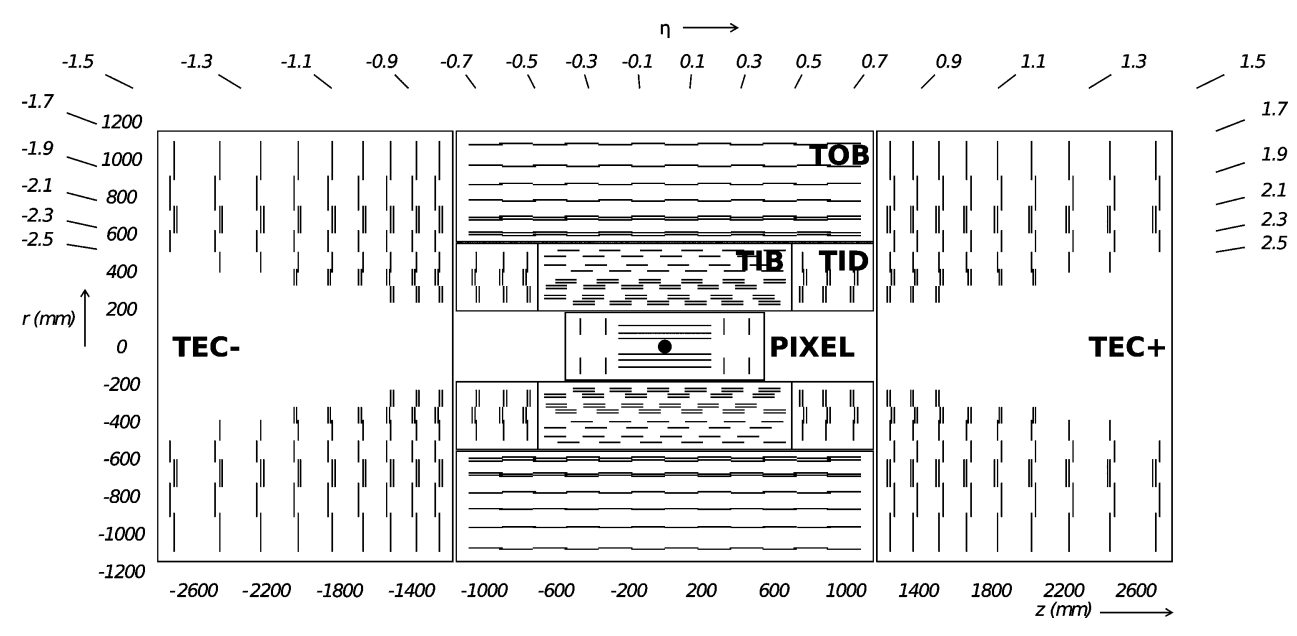


the disk planes to produce azimuthal charge sharing and radial Lorentz drift, which enhances the radial charge sharing. The charge sharing improves the endcap resolution in both planes.

The CMS silicon strip detector has 9.3 million active elements instrumenting a surface area of $198 \mathrm{~m}^{2}$. The detector consists of three large subsystems. The Tracker Inner Barrel and Disks (TIB/TID) extend in radius to $55 \mathrm{~cm}$ and are composed of four barrel layers, supplemented by three disks at each end. The TIB/TID delivers up to four $r-\phi$ measurements on a trajectory using $320 \mu \mathrm{m}$ thick silicon microstrip sensors, which have their strips oriented parallel to the beam axis in the barrel and oriented radially in the disks. The strip pitch is $80 \mu \mathrm{m}$ in the inner pair of TIB layers and $120 \mu \mathrm{m}$ in the outer pair of TIB layers. In the TID, the mean pitch varies between $100 \mu \mathrm{m}$ and $141 \mu \mathrm{m}$. The TIB/TID is enclosed within the Tracker Outer Barrel (TOB), which has an outer radius of $116 \mathrm{~cm}$. The TOB consists of six barrel layers of $500 \mu \mathrm{m}$ thick microstrip sensors with strip pitches of $183 \mu \mathrm{m}$ in the first four layers and $122 \mu \mathrm{m}$ in the last pair of layers. The TOB extends to $\pm 118 \mathrm{~cm}$ in $z$. Beyond this $z$ range, the Tracker EndCaps (TEC) instrument the region $124<|z|<280 \mathrm{~cm}$ and $22.0<r<113.5 \mathrm{~cm}$. Each TEC is composed of nine disks that are instrumented with up to seven rings of radial strip silicon detectors. The sensor thicknesses are thin $(320 \mu \mathrm{m})$ in the inner four rings and thick $(500 \mu \mathrm{m})$ in the outer three rings; the average radial strip pitch varies from $97 \mu \mathrm{m}$ to $184 \mu \mathrm{m}$. The inner two layers of the TIB and TOB, the inner two rings of the TID and TEC, and the fifth ring of the TEC include a second microstrip detector module that is mounted back-to-back at a stereo angle of $100 \mathrm{mrad}$ and that enables a measurement of the orthogonal coordinate. Assuming fully efficient planes and not counting hits in stereo modules, there are from eight to 14 high precision measurements of track impact points for $|\eta|<2.4$.

\section{Data samples}

The results presented in this paper were obtained from data samples collected by the CMS experiment during LHC operation in December 2009 at proton-proton centre-of-mass energies of 0.9 and $2.36 \mathrm{TeV}$. The CMS axial magnetic field was maintained at the nominal value of $3.8 \mathrm{~T}$ and the silicon pixel and silicon strip detectors were biased at their nominal voltages. Due to the relatively low LHC luminosity, the CMS readout was triggered by the coincidence of signals from the beam scintillator counter (BSC) minimum bias trigger and the beam pick-up timing detector which detects the passage of the beam bunches [2]. The BSC minimum bias trigger requires that the arrival times of the signals from the forward and backward arms of the BSC $(3.23<|\eta|<4.65)$ be consistent with the passage of particles emerging from a pp collision in the middle of CMS. In contrast, the BSC beam-gas trigger, used to veto noncollision events, requires that the arrival times be consistent with the passage of particles traversing the detector from one end to the other in time with particles from either beam. The total number of selected minimum bias events is approximately 305000 .

Prior to the LHC pp collisions, the CMS experiment was commissioned using events containing cosmic muons during Cosmic Run At Four Tesla (CRAFT) [3]. The detector and magnetic field conditions during CRAFT were quite similar to the conditions during pp collisions. Thus, the results obtained from CRAFT provided good initial operating points for the pixel detector [4], the strip detector [5], the tracker alignment [6], and the magnetic field [7]. The data used in the referenced CRAFT papers were obtained in the fall of 2008, more than one year before the pp collisions. In most cases, more recent CRAFT data were used to improve on these results.

\section{Tracker commissioning}

The following two subsections describe the operating characteristics and performance of the silicon pixel and silicon strip detectors, respectively.

\subsection{Silicon pixel detector}

\subsubsection{Operating conditions}

In order to make maximal use of experience gained from the operation of the pixel detector with cosmic rays during summer/autumn 2009, the operating conditions were not changed for the December 2009 data taking period. The coolant temperature was kept constant at $7^{\circ} \mathrm{C}$. The bias potential applied to the $285 \mu \mathrm{m}$ thick p-spray barrel sensors [8] was a uniform $150 \mathrm{~V}$. The bias potential applied to the $270 \mu \mathrm{m}$ thick p-stop endcap sensors [9] was a uniform $300 \mathrm{~V}$. Small fractions of the barrel (1.0\%) and endcap (3.1\%) detectors were inactive resulting in a net operational fraction of $98.4 \%$ for the entire detector.

The calibration procedures described in Ref. [4] were used to determine the ADC gains and pedestals for all channels. Iterative tuning reduced the mean (spread) of the readout threshold distributions for the pixel Readout Chips (ROCs) from the values measured during the 2008 cosmic ray commissioning [4] to $2733 e$ (196e) in the barrel detector and $2483 e(163 e)$ in the endcap detectors, where $e$ is the magnitude of the electron charge. These measured threshold values apply only to the calibration procedure. Because the 
bandwidth of the preamplifiers is limited by power considerations, small signals can take more than a bunch crossing time ( $25 \mathrm{~ns}$ ) to fire the zero-crossing discriminator that triggers the storage of the signal. This causes some smaller signals to be associated with the wrong bunch crossing and to be ignored by the readout system. The net result is that the effective or "in-time" thresholds are larger than the set values.

The effective thresholds are estimated by comparing the distribution of measured cluster $x$-sizes (azimuthal direction in the barrel detector and radial direction in the endcap detectors) with those predicted by the detailed pixel simulation, PIXELAV $[10,11]$. The cluster sizes are sensitive to the effective thresholds. To avoid highly ionizing particles, the tracks used in this analysis were required to have momenta larger than $4 \mathrm{GeV} / c$. This selection ensures that even protons and deuterons produce signals that are within a few percent of the ionization minimum. By varying the simulated thresholds until the measured and simulated distributions agree, the average effective thresholds are found to be approximately $3500 e$ in the barrel detector and $3000 e$ in the endcap detectors.

A study of the pixel hit reconstruction efficiency using a technique similar to the strip detector technique described in Sect. 4.2.4 suggests that the efficiency is larger than $99 \%$ for the live regions of the detector and is consistent with earlier work [2].

\subsubsection{Pixel timing scan}

The pixel detector readout system uses the $40 \mathrm{MHz}$ LHC clock as input. Signals from the CMS trigger system must arrive at the correct time within the $25 \mathrm{~ns}$ clock cycle to associate the correct bunch crossing time stamp with any signal above the readout threshold. An optimally phased clock signal will maximize the number of pixels observed in clusters. The overall trigger timing was adjusted by varying the clock phase until the average barrel and endcap cluster sizes as measured in minimum bias triggers were maximized. These quantities are plotted versus clock phase in Fig. 2. The clock phase setting of $6 \mathrm{~ns}$ was found to optimize the smoothly varying detector averages. A finer module-by-module adjustment of the clock phase will be performed when higher trigger rates become available.

\subsubsection{Operating characteristics with minimum bias triggers}

The distributions of the number of clusters observed in $0.9 \mathrm{TeV}$ events selected by the minimum bias trigger are shown in Fig. 3. The observed data, shown as solid dots, are compared with fully simulated data, shown as histograms, that were generated with a recent tuning of the PYTHIA event generator [12]. The left plot shows the distribution for all events, whereas the right plot shows the distribution after removing events that also satisfy the beam-gas trigger. There is an excess of large multiplicity events that are removed by the beam-gas trigger requirement. The source of these events could be beam-gas interactions or beam scraping in

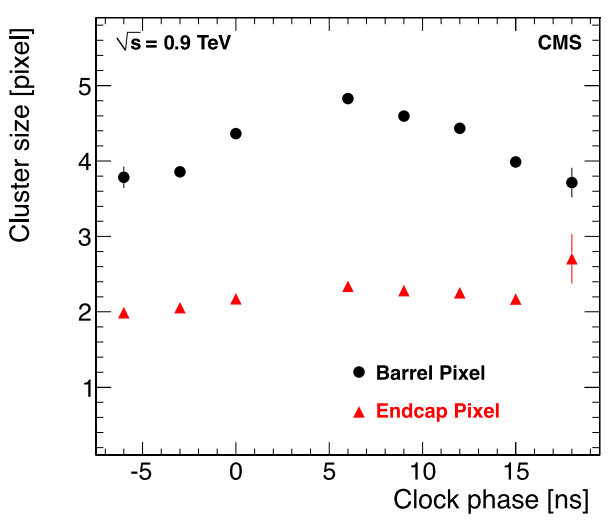

Fig. 2 The average cluster size distributions for the barrel and endcap pixel detectors in minimum bias events are plotted versus clock phase
Fig. 3 The cluster multiplicity of (a) all minimum bias triggered events and (b) those that do not trigger the beam-gas veto in the $0.9 \mathrm{TeV}$ data sample. The histograms show the similar distribution for a sample of simulated data

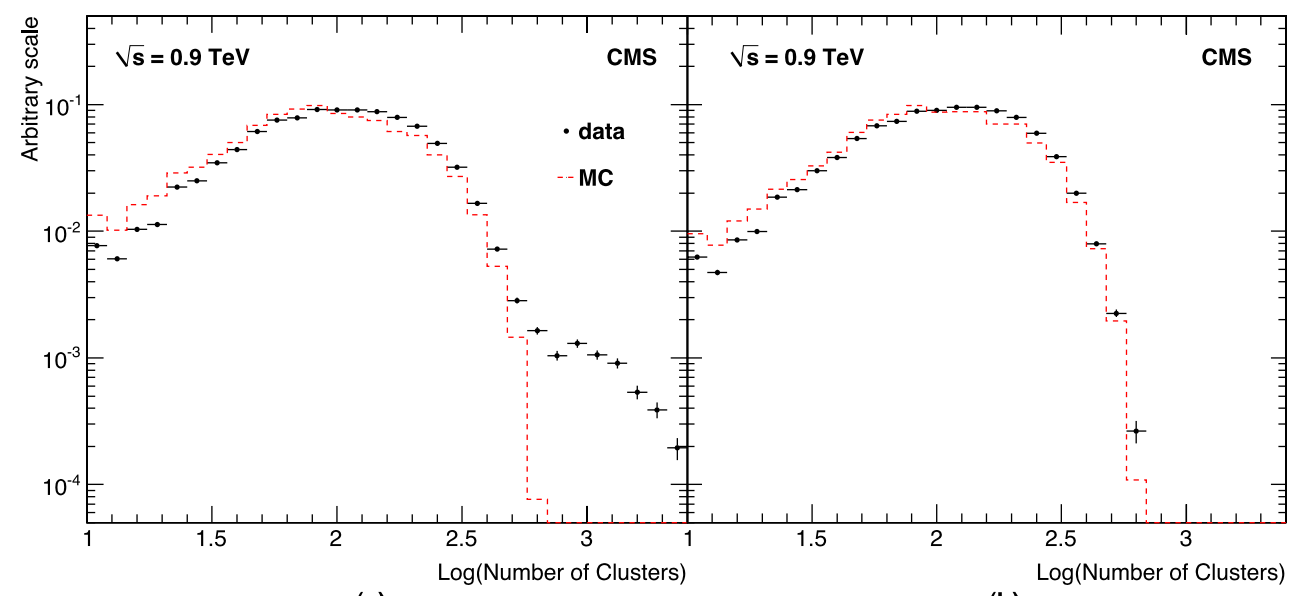

(a) (b) 
Fig. 4 The normalized cluster charge measured in the (a) barrel and (b) endcap pixel detectors for the sample of $0.9 \mathrm{TeV}$ minimum bias events. The insets show the same distributions on semi-log scales

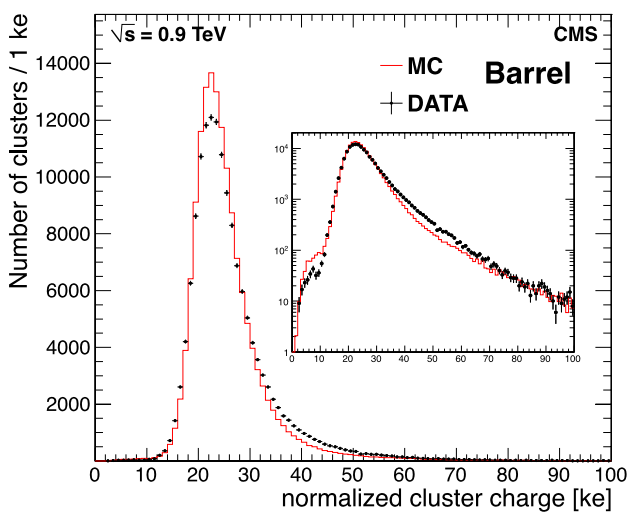

(a)

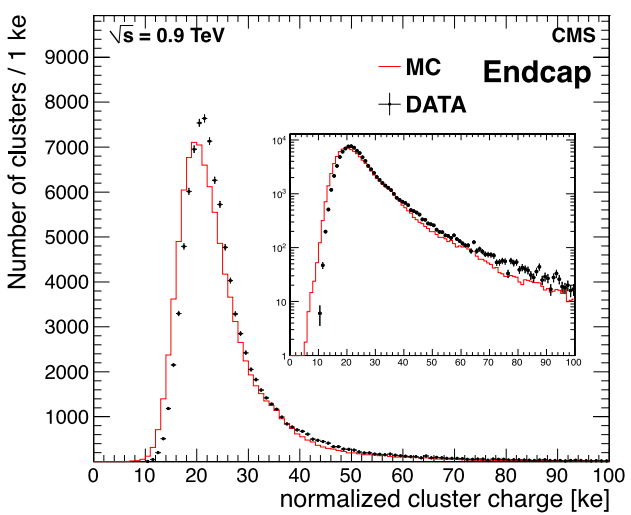

(b)
Table 1 The average cluster multiplicity per layer/disk in $0.9 \mathrm{TeV}$ minimum bias triggers. The simulation errors are entirely statistical and do not represent the uncertainties in the event modelling. The asymmetry seen in the forward and backward endcaps is caused by an offset in the luminous region along the beam axis

\begin{tabular}{|c|c|c|c|c|c|}
\hline \multicolumn{3}{|c|}{ Barrel Pixel: clusters/layer } & \multicolumn{3}{|c|}{ Endcap Pixel: clusters/disk } \\
\hline Layer & Measured & Simulation & Disk & Measured & Simulation \\
\hline 1 & $35.2 \pm 0.9$ & $31.6 \pm 1.2$ & -2 & $8.0 \pm 0.1$ & $7.3 \pm 0.2$ \\
\hline 2 & $30.6 \pm 0.8$ & $27.8 \pm 1.1$ & -1 & $7.8 \pm 0.1$ & $7.2 \pm 0.2$ \\
\hline \multirow[t]{2}{*}{3} & $27.4 \pm 0.8$ & $24.8 \pm 1.0$ & 1 & $8.1 \pm 0.1$ & $7.7 \pm 0.2$ \\
\hline & & & 2 & $8.6 \pm 0.1$ & $8.1 \pm 0.2$ \\
\hline
\end{tabular}

the beam transport system near the interaction point. After removal of the beam background events, the measured distributions are approximately consistent with preliminary expectations. The measured average cluster multiplicities per layer (barrel detector) and per disk (endcap detector) are listed in Table 1. They are compared with the expectation from the simulation and are found to be in rough agreement. It should be noted that the event generator is based on an event model that has not yet been tuned in detail and is not expected to provide accurate predictions.

During the extremely low luminosity run in December 2009 (the instantaneous luminosity was typically in the range $10^{26}-10^{27} \mathrm{~cm}^{-2} \mathrm{~s}^{-1}$ ), the beam background events occurred at a rate that was roughly comparable to the rate of minimum bias triggers. Because they are characterized by particle trajectories that are nearly parallel to one of the beams, most background events ( $\sim 90 \%)$ do not fire the minimum bias trigger but do have clusters in the endcap detectors and elongated clusters in the first two layers of the barrel detector. At the beam energies of the December 2009 run, the pixel detector occupancies associated with the background events were typically five times larger than those associated with minimum bias events. The beam-gas trigger veto effectively removes background events, as do cluster shape, track quality, and vertex requirements.
The cluster charge distributions measured in the barrel and endcap detectors with the $0.9 \mathrm{TeV}$ sample are shown as solid dots in Fig. 4. Each entry is scaled by the ratio of the pixel sensor thickness to the track path length in the sensor. The solid histograms represent the expectations from the PYTHIA-based, full detector simulation. The measured and simulated barrel distributions have similar peaks but the measured distribution is somewhat broader than the simulated one. This may be due to residual pixel-to-pixel gain variation resulting from the use of a single gain for all 80 channels in each ROC column or residual module-to-module clock phase variation. The corresponding distributions for the endcap detectors have similar widths but indicate a 5\% charge-scale mismatch.

\subsubsection{Lorentz angle calibration}

The use of n-in-n pixel technology and the large magnetic field in CMS imply that pixel hit reconstruction involves large Lorentz drift corrections (the typical bias corrections are $53 \mu \mathrm{m}$ in the barrel and $10 \mu \mathrm{m}$ in the endcap). The estimation of track impact coordinates from pixel clusters is performed with two different algorithms. The simpler, faster "Generic Algorithm" [13] uses the Lorentz width $W_{L}$ to estimate the projected cluster size and bias correction. The Lorentz width is the product of the effective thickness of the sensor $T_{\text {eff }}$ and the tangent of the average Lorentz angle $\theta_{L}: W_{L}=T_{\text {eff }} \tan \theta_{L}$. Due to the focussing of the electric field at the $\mathrm{n}+$ implants, the charge sharing near the $\mathrm{n}+$ side of the sensors is reduced. This is modelled by the effective thickness which is $5-10 \%$ smaller than the physical thickness of the sensor substrate. The detailed PIXELAV simulation is used to extract the Lorentz width by applying the Generic Algorithm to a sample of simulated clusters and by adjusting $W_{L}$ to minimize the bias and maximize the resolution. The slower, more sophisticated "Template Algorithm" [14] fits pre-computed cluster shapes to the measured clusters. The Lorentz drift effects are encoded in the cluster shapes and the same PIXELAV simulation is 


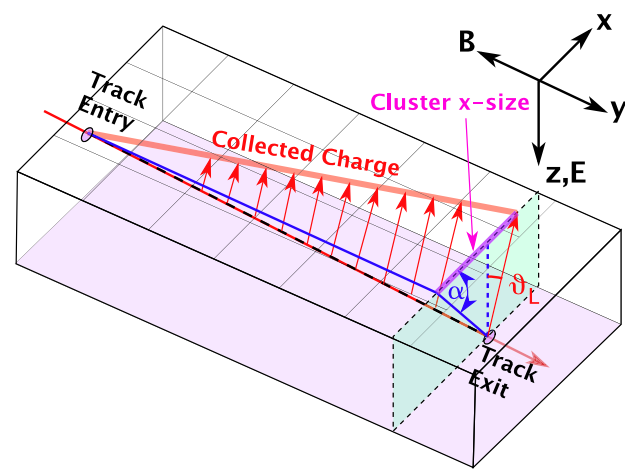

(a)

Fig. 5 (a) The pixel local coordinate system and track angle definitions. The local $z$ axis coincides with the sensor electric field $\vec{E}$. The local $x$ axis is chosen to be parallel to $\vec{E} \times \vec{B}$ where $\vec{B}$ is the axial magnetic field. The local $y$ axis is defined to make a right-handed coordinate system. The angle $\alpha$ is the angle between the $x$ axis and the

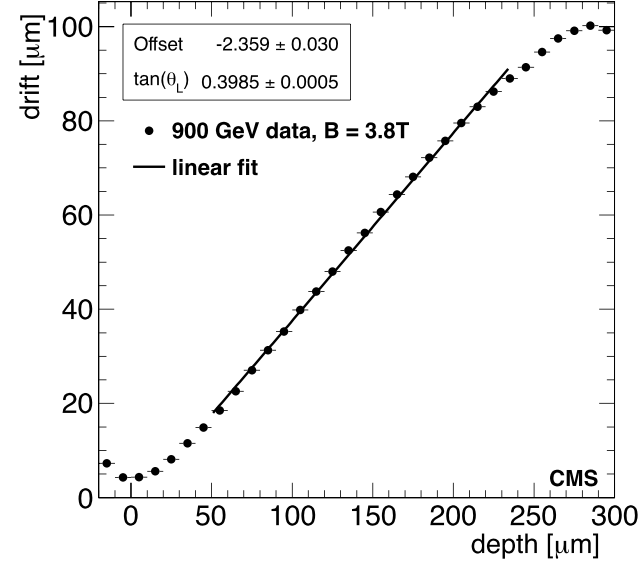

(b)

track projection on the local $x z$ plane. (b) The transverse cluster displacement of highly inclined barrel clusters as a function of depth for a sample of $0.9 \mathrm{TeV}$ minimum bias events at a magnetic field of $3.8 \mathrm{~T}$. The tangent of the Lorentz angle is given by the slope of a linear fit which is shown as the solid line
Table 2 The tangent of the Lorentz angle $\tan \theta_{L}$ as determined by 2009 calibrations

\begin{tabular}{lllll}
\hline \multicolumn{2}{l}{ 2009 Lorentz Angle Measurements } & & \\
\hline Sample & Detector & Technique & Measured $\tan \theta_{L}$ & Simulation \\
\hline Cosmic Ray & Barrel & Cluster Size & $0.409 \pm 0.002$ (stat) & $0.407 \pm 0.002$ (stat) \\
Cosmic Ray & Endcap & Cluster Size & $0.081 \pm 0.005$ (stat) & $0.080 \pm 0.004$ (stat) \\
Minimum Bias & Barrel & Grazing Angle & $0.3985 \pm 0.0005$ (stat) & $0.4006 \pm 0.0005$ (stat) \\
Minimum Bias & Barrel & Cluster Size & $0.409 \pm 0.002$ (stat) & $0.411 \pm 0.005$ (stat) \\
\hline
\end{tabular}

used to compute them. Therefore, the actual Lorentz calibration procedure is to tune the detailed simulation to agree with data and then to generate a Lorentz width for the Generic Algorithm and cluster shapes for the Template Algorithm.

Two different techniques have been used to perform the calibration. The 2008 cosmic ray data were calibrated by measuring the cluster $x$-sizes as functions of $\cot \alpha$ (see Fig. 5 a for definitions) and by determining the locations of the cluster size minimum $\cot \alpha_{\min }$ [4]. In the pixel barrel, $-\cot \alpha_{\min }$ is equal to $\tan \theta_{L}=r_{H} \bar{\mu} B$, where $r_{H}$ is the electron Hall factor, $\bar{\mu}$ is the average electron mobility, and $B$ is the magnetic field. The 2008 cosmic ray measurements suggested that the value of the electron Hall factor used in PIXELAV should be increased to 1.05 from the 1.02 value determined in test beam measurements [15]. In 2009, the temperature of the detector was lowered and the bias voltage of the pixel barrel was increased, which changed the average Lorentz angles in both barrel and endcap detectors. New cosmic ray based determinations are reported in Table 2 and are compared with the tuned simulation.

The barrel calibration was repeated with collision data in December 2009 using a new "grazing angle" technique
[16]. This technique makes use of the two-dimensional pixel segmentation to simultaneously measure the average transverse displacement of the charge carriers as a function of distance along clusters produced by a sample of highly inclined tracks. Since longitudinal position in the cluster is completely correlated with depth in the junction, this technique determines the average transverse carrier displacement as a function of depth as shown graphically in Fig. 5b. The average Lorentz angle, extracted from the linear fit shown in the figure, is compared with the detailed simulation in Table 2. The extremely large population of highly curved, low transverse momentum tracks observed in minimum bias triggers spans the $\cot \alpha$ region needed to determine the minimum projected cluster size in the pixel barrel. This enables the use of the cluster size technique as a cross check which is also reported in Table 2 . Note that the two techniques are affected by different systematic effects and that a better than $1 \%$ consistency is observed between the real and simulated measurements in all cases. A variation of fitting procedures suggests that the total systematic uncertainty on the Lorentz angle calibration is less than $2 \%$. 


\subsubsection{Resolution study}

The intrinsic position resolution in a limited range of the angular acceptance was measured using tracks from minimum bias triggers that traverse overlapping sensors in the barrel layers. A similar analysis was performed in a very different angular region with 2008 cosmic ray data [4] using the measurement technique given in Ref. [17]. Tracks passing through two overlapping modules in the same layer are used to compare the hit position with the expected position from the track trajectory. Because it is insensitive to alignment uncertainties, the difference of the local track impact points on a fitted trajectory is known about ten times more precisely than are the individual predicted hit positions. A double difference is formed by taking the difference between the measured hit position difference in the two modules and the predicted trajectory position difference. The width of this double difference distribution is insensitive to translational misalignment of the overlapping modules.

To limit the effect of multiple scattering, a minimum track momentum of $2.5 \mathrm{GeV} / \mathrm{c}$ is required. Clusters with measured charge below $10000 e$ or containing pixels on the sensor edges are excluded. The double difference widths are fitted with a Gaussian and the uncertainty from the trajectory prediction is subtracted quadratically to recover the hit resolution on the position difference. With the assumption of equal resolution for each of the modules in the overlap, the final fit values for the resolution for a single module are $12.7 \pm 1.0 \mu \mathrm{m}$ along $x$ and $32.4 \pm 1.5 \mu \mathrm{m}$ along $y$. The PIXELAV simulation is used to generate a sample of clusters that has the same distribution of impact angles as the measured sample. Since the simulation does not include the doublesize pixels that span the gaps between the 16 readout chips which tile each module, a subsample of the overlap data sample is used to determine single-size-pixel resolutions of $12.6 \pm 1.6 \mu \mathrm{m}$ along $x$ and $28.1 \pm 2.4 \mu \mathrm{m}$ along $y$. These numbers can be directly compared with those extracted from Gaussian fits to the simulated residual distributions. The simulated resolutions are $14.1 \pm 0.5 \mu \mathrm{m}$ and $24.1 \pm 0.5 \mu \mathrm{m}$ along $x$ and $y$, respectively, and agree reasonably well with the measured resolutions. Because overlaps occur only at the edges of the track $\alpha$-angle acceptance where the $x$ sizes of the clusters deviate from the optimal size of two, the measured and simulated $x$ resolutions are somewhat worse than the typical $x$ resolution (less than $10 \mu \mathrm{m}$ ) expected for most collision-related clusters. The measured and simulated $y$ resolutions are expected to be typical of the detector performance.

\subsection{Silicon strip detector}

\subsubsection{Operating conditions}

All of the modules in the strip tracker were biased at $300 \mathrm{~V}$ in the early collision running. This is the same setting that was used in the CRAFT studies and is well above the full depletion voltage for the sensors. Similarly, the coolant temperature was set at $4-6^{\circ} \mathrm{C}$, the same as in the CRAFT study. This meant that the $p^{+}$on $n$ sensors [18] were approximately at room temperature.

There are two main modes of operation for the strip tracker analogue pipeline integrated circuits (APV25 [19]): peak and deconvolution [20, 21]. In deconvolution mode, the output charge for each strip represents a weighted sum of three consecutive pipeline cells [22]. Although deconvolution mode was designed to avoid signal pile-up in high (design) luminosity operations, it will be necessary to run in this mode whenever the expected separation between proton bunches will be less than a few hundred nanoseconds. The luminosity in the early collision running was very low and the bunches well separated; most of the strip data were collected in peak mode, which is based on the signal in a single pipeline cell. All of the data, whether in peak or deconvolution mode, were zero suppressed, meaning that only strips which were part of clusters were read out for each event.

Many of the starting parameters for the strip tracker during the early collision running had been established in the preceding CRAFT period. For example, the timing of the tracker subsystems (in peak mode) with respect to CMS triggers was set during the cosmic ray muon studies. Similarly, the alignment parameters for the strip detector modules were derived from the same studies.

As part of the alignment process, offsets had been determined for cluster positions in sensors due to the Lorentz drift of holes and electrons under the influence of the solenoid field. For barrel layers, the Lorentz angle correction for cluster positions during track reconstruction is about $10 \mu \mathrm{m}$, which is significantly larger than the $3-4 \mu \mathrm{m}$ alignment precision achieved in the cosmic ray studies [6].

\subsubsection{Strip timing scan}

As the strip tracker was operated in peak mode at the start of the early collision running, the trigger timing established in the preceding CRAFT period could be used. In CRAFT the sampling time of the APV25's was set within each subsystem by means of a dedicated synchronization signal, adjusted according to the measured readout fibre lengths. The synchronization of the subsystems was obtained using the signal from cosmic ray muon tracks. Details on how the scan was done can be found in Ref. [23]. Toward the end of the data collection period the APV25 mode was changed from peak to deconvolution and since timing is more critical in the latter, a fine-delay scan was made following the mode change. For expediency only one layer (TOB L3) was used in the study.

Figure 6 shows the result of the fine-delay timing scan. The timing adjustment for the clock and trigger signals is set 
on the front-end hybrids and the smallest step size is $1.04 \mathrm{~ns}$. From the figure it can be seen that the timing prior to the scan had been off by about $10 \mathrm{~ns}$ from ideal. This level of mistiming resulted in an estimated 2.5\% decrease in Signalto-Noise $(\mathrm{S} / \mathrm{N})$ in the strip modules during the peak mode running, where the delay timing is less critical. The amplitude that is measured in the timing scan represents the signal of the highest pulse height strip in a cluster scaled by the ratio of the sensor thickness to the path length of the track in the sensor.

Following the scan, the timing offsets for all of the strip tracker subsystems were updated and some data were collected in deconvolution mode. No data samples were collected in peak mode with the new delays.

\subsubsection{Signal-to-noise measurements}

Signal-to-Noise measurements were made in both peak and deconvolution modes. In peak mode, the $\mathrm{S} / \mathrm{N}$ ratio was determined at both centre-of-mass energies, 0.9 and $2.36 \mathrm{TeV}$, whereas deconvolution mode is restricted to $2.36 \mathrm{TeV}$. The ratio is evaluated on the basis of charge clusters associated

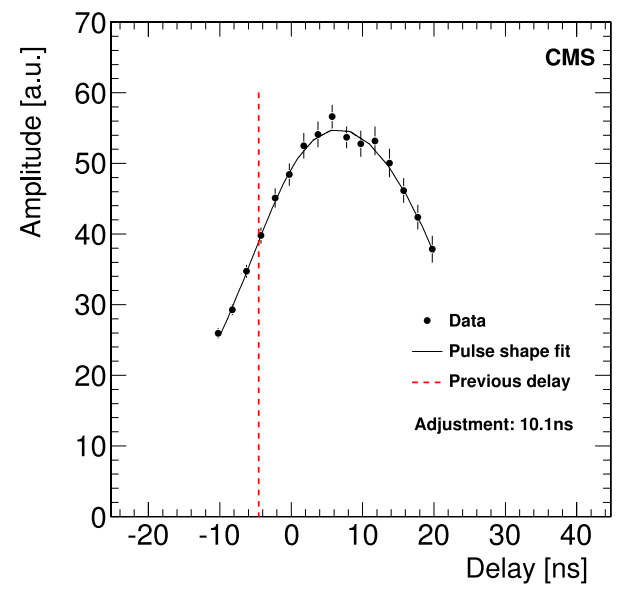

Fig. 6 Normal-incidence-scaled charge (arbitrary units) of the highest pulse height strip in a cluster as a function of the readout delay with respect to the CMS trigger, in deconvolution mode. The dashed vertical line corresponds to the setting prior to the timing scan with reconstructed tracks, where the individual strip noise values are taken from calibration runs. For track angles that are not normal to the surface of modules the signal values are scaled by the cosine of the angle relative to the local normal. This is done to give the same expectation value per cluster for modules of the same type. Cluster noise, which takes into account the noise of each strip within a cluster, is used as the denominator in the $\mathrm{S} / \mathrm{N}$ ratio. When all strips within a cluster have the same noise, cluster noise is equivalent to the noise of a single strip. Further details on the determination of the $\mathrm{S} / \mathrm{N}$ ratio can be found in Ref. [24].

Figures $7 \mathrm{a}$ and $7 \mathrm{~b}$ show the $\mathrm{S} / \mathrm{N}$ distributions for the TIB and TOB modules, respectively, in deconvolution mode. Included with each distribution is the result of the fit to a Landau distribution convolved with a Gaussian distribution. The most probable value of the fitted curves is taken to be the $\mathrm{S} / \mathrm{N}$ value and results for all of the strip tracker subdetectors are summarized in Table 3 for all three running conditions. Peak values shown in the table have not been corrected for the $2.5 \%$ loss due to non-optimal timing. They are comparable with results obtained in the CRAFT studies and in earlier cosmic ray studies. The difference in peak and deconvolution mode $\mathrm{S} / \mathrm{N}$ values stems largely from the higher noise in deconvolution. After calibration there is some variation in signal values (measured in electrons) for the two modes, but this has been shown to be within $10 \%$. The $\mathrm{S} / \mathrm{N}$ ratio should not depend on the centre-of-mass energy and this is confirmed by the table entries.

Although it is not possible to directly compare channel noise distributions in the early collision data with results

Table 3 Summary of strip tracker Signal-to-Noise measurements. The peak mode ratios have not been corrected for the estimated $2.5 \%$ decrease in signal from the trigger mistiming, as described in the text

\begin{tabular}{lcccll}
\hline Conditions & TIB & TID & TOB & TEC thin & TEC thick \\
\hline 0.9 TeV, peak mode & 27.4 & 26.7 & 34.1 & 28.8 & 35.7 \\
2.36 TeV, peak mode & 27.4 & 26.8 & 34.1 & 28.8 & 35.7 \\
$2.36 \mathrm{TeV}$, deco mode & 20.3 & 19.2 & 23.9 & 20.3 & 26.1 \\
\hline
\end{tabular}

Fig. 7 Signal-to-Noise distributions in deconvolution mode for (a) (thin sensor) TIB and (b) (thick sensor) TOB modules. The curves are results of the fits to a Landau distribution convoluted with a Gaussian distribution

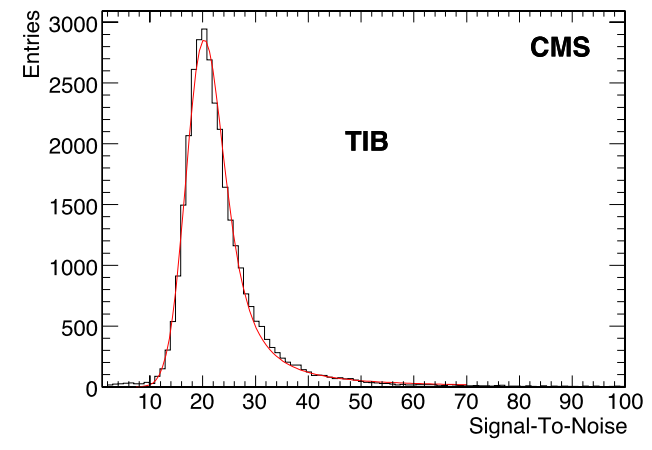

(a)

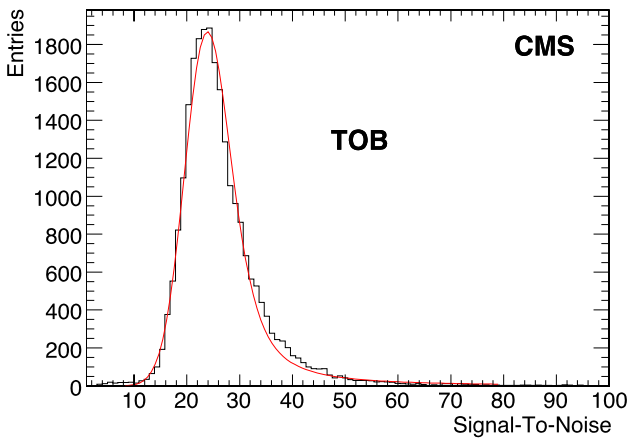

(b) 
from calibration runs given the zero suppression, the frequency and distribution of clusters in empty LHC buckets provide an indirect cross check of the calibration results and assumptions about the Gaussian and uncorrelated nature of the noise. For example, with bad modules excluded from the readout the mean number of clusters in empty buckets, out of some 9 million channels, was 4.2. This is consistent with the clustering rules, which require a certain number of standard deviations (five for the total charge in a cluster), and Gaussian probabilities. By way of contrast, there were $\sim 1200$ clusters per minimum bias trigger in the $0.9 \mathrm{TeV}$ data.

\subsubsection{Strip layer efficiencies}

Efficiencies for strip tracker layers were determined using events that were collected in peak mode. Reconstructed tracks in these events were required to have a minimum of eight hits in order to be used in the efficiency measurements. To avoid inactive regions and allow for alignment imprecision, trajectories passing near the edges of sensors were excluded. The presence of a hit anywhere within the non-excluded region of a traversed module was counted as a positive response; efficiency is determined by the ratio of positive responses to the total number of traversing tracks. Layers under study were not removed from the track reconstruction and could in fact count toward the minimum hit requirement. The total integrated hit efficiency during the early collision period was measured to be $97.8 \%$, which is essentially explained by the number of bad modules in the strip tracker. That is, about $2.2 \%$ of the modules have been excluded from the readout because of problems with high voltage short circuits, control ring failures, or other issues. With known problem modules excluded, the overall hit efficiency is $99.8 \%$, consistent with the $\sim 0.2 \%$ bad channel rate from the construction process. Detailed simulations, used to determine track reconstruction efficiency, take into account inactive regions in addition to the measured efficiencies. The efficiency measurements for the collision data include an estimated $0.04 \%$ systematic error due to the use of the layers under study in the reconstruction process and the wide search windows within modules.

\subsubsection{Energy loss measurement}

Although the primary function of the strip tracker is to provide hit position information for track reconstruction and precise momentum determination, the wide linear range of the strip channel output also provides a measure of energy loss. That is, the charge collected in a hit cluster is directly proportional to energy lost by a particle, largely through ionization, while traversing the silicon. For reconstructed tracks the angle $\theta$ between the track direction and the axis normal to module sensor is well defined for each hit on the track. The instantaneous energy loss per unit path length $(d E / d x)$ in the silicon is then approximated by the quantity $\Delta E /(\Delta L \cdot \sec \theta)$, where $\Delta E$ is the cluster charge expressed in units of $\mathrm{MeV}$ and $\Delta L$ is the normal-angle thickness of the active volume of the silicon sensor. All of the TIB and TID modules and the modules on rings 1-4 of the TEC have silicon sensors that are $320 \mu \mathrm{m}$ thick, whereas the TOB and TEC ring 5-7 modules have $500 \mu \mathrm{m}$ thick sensors. Some $30 \mu \mathrm{m}$ of the nominal thicknesses for both thin and thick types is inactive material, i.e., does not contribute to the charge collection.

In zero-suppressed readout, which was used exclusively in the early collision period, there are eight ADC bits for the charge on each channel within a cluster. Channel gains are set such that a single ADC count corresponds to about one-quarter of the average noise and full scale corresponds to approximately three times the average loss expected from normally incident minimum ionizing particles. The highest two ADC values have a special significance: 254 implies a value between 254 and 1024 counts, and 255 indicates that the actual value was in excess of 1024 counts. The $d E / d x$ algorithm includes the saturated values but without any special treatment.

The main point in determining energy loss per unit path length is that, for a given medium, $d E / d x$ depends largely on the velocity $(\beta)$ of the traversing particle. By combining $d E / d x$ information with the measured momentum $p$ of a track, one can determine the mass of the traversing particle. On the scale of charged particle momenta in CMS collisions, there is only a limited range near the low end where the difference in $\beta$ values is significant enough to distinguish among long-lived hadrons. The momentum range where pions would have relatively large energy loss is such that tracks tend to curl up in the $3.8 \mathrm{~T}$ solenoid field and thus fail to be reconstructed.

The strip hits on reconstructed tracks represent independent measures of $d E / d x$, ignoring the negligible loss of energy in traversing the tracker. Although pixel hits are included in the track reconstruction, they are not used in the $d E / d x$ calculation due to their more limited linear range. Several methods have been used to determine an estimate for the most probable $d E / d x$ value based on the measurements in the strip tracker modules traversed by a track. One example, the Harmonic-2 estimator [25], is defined by

$\frac{d E}{d x}=\left(\frac{1}{N} \sum_{i=1}^{N} \frac{1}{c_{i}^{2}}\right)^{-1 / 2}$,

where $c_{i}$ is the charge, per unit path length of silicon, of the $i$ th hit associated to the track. Figure 8 shows the relationship between the Harmonic- $2 d E / d x$ estimator and momentum for $0.9 \mathrm{TeV}$ data taken in peak mode. In the figure, clear bands can be seen for kaons and protons and to a much lesser extent for deuterons. 
Fig. 8 Energy loss versus the momentum of tracks (a) and frequency of tracks as a function of track mass as determined from the measured energy loss and momentum (b). The lightly shaded line in (a) indicates the fit in the reference range of the proton band while the darker lines correspond to extrapolations for kaons, protons, and deuterons based on the fit parameters

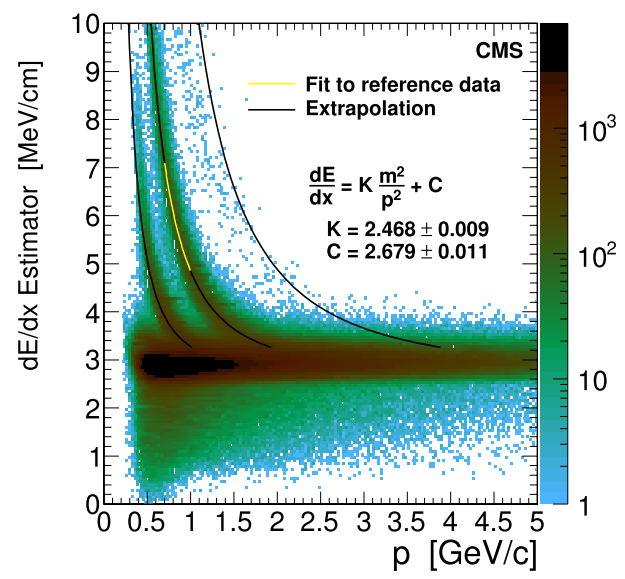

(a)

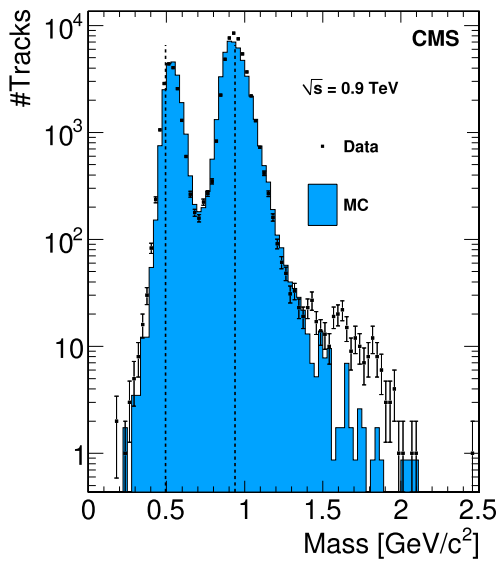

(b)
An estimate of the mass of each candidate can be obtained using the particle momentum and the measurement of the ionization energy loss provided by the $d E / d x$ estimators. To this end the following relation between $d E / d x$, $p$, and $m$ is assumed for the momenta below the minimum ionizing region:

$\frac{d E}{d x}=K \frac{m^{2}}{p^{2}}+C$.

The proton line in Fig. 8a is used to extract the parameters $K$ and $C$ in (2). The $0.7-1.0 \mathrm{GeV} / c$ range in the proton band is used for the reference data fit, while extrapolations based on the same $K$ and $C$ values yield a good agreement for protons with momenta above and below the reference range and for kaons.

The mass spectrum that results from inverting (2) for all tracks with $d E / d x>4.15 \mathrm{MeV} / \mathrm{cm}$ and $p<2 \mathrm{GeV} / c$ is shown in Fig. 8b. From the frequency plot one can observe clear kaon and proton peaks as well as good agreement for the peaks from a Monte Carlo simulation. There is also evidence for a deuteron peak in data, although saturation of the ADC scale is particularly pronounced for deuterons given their reduced $\beta$ values and relatively higher $|\eta|$ values. That the deuteron peak is poorly modelled by the simulation is partly understood as the underlying generator, PYTHIA, does not produce deuterons by design, although they can be produced in the subsequent GEANT [26] hadron showers.

\section{Track reconstruction}

The track reconstruction algorithms rely on a good estimate of the proton-proton interaction region, referred to as the beamspot. The beamspot is used as a precise estimate of the primary interaction point (in the transverse direction) prior to primary vertex reconstruction and as the sole primary interaction point if no primary vertex is found. When the beamspot centre is displaced from the expected position there is a correlation between the transverse impact parameter $\left(d_{x y}\right)$ and the angle of the track at the point of closest approach $\left(\phi_{0}\right)$. The beamspot fitter [27] uses an iterative $\chi^{2}$ fitter to exploit this correlation between $d_{x y}$ and $\phi_{0}$, looping over a sample of reconstructed tracks (using the old beamspot) to determine the new beamspot parameters. After the beamspot is measured, the standard track reconstruction is performed. During the 2009 data taking, a beamspot was fitted during each LHC fill; fill-to-fill variations were at the level of $\sim 0.5 \mathrm{~mm}$ in $x$ and $y$, and $\sim 2 \mathrm{~cm}$ in $z$.

Starting from the location of the beamspot, an initial round of track and vertex reconstruction is performed using only pixel hits. The pixel vertices found at this stage are used in the standard tracking. The standard track reconstruction at CMS is performed by the combinatorial track finder (CTF) [28]. Tracks are seeded from either triplets of hits in the tracker or pairs of hits with an additional constraint from the beamspot or a pixel vertex, yielding an initial estimate of the trajectory, including its uncertainty. The seed is then propagated outward in a search for compatible hits. As hits are found, they are added to the trajectory and the track parameters and uncertainties are updated. This search continues until either the boundary of the tracker is reached or no more compatible hits can be found. An additional search for hits is performed starting from the outermost hits and propagating inward. In the final step, the collection of hits is fit to obtain the best estimate of the track parameters.

The current implementation of the CTF performs six iterations. Between each iteration, hits that can be unambiguously assigned to tracks in the previous iteration are removed from the collection of tracker hits to create a smaller collection that can be used in the subsequent iteration. At the end of each iteration, the reconstructed tracks are filtered to remove tracks that are likely fakes and to provide a means of quantifying the quality of the remaining tracks. The filtering uses information on the number of hits, the nor- 
malized $\chi^{2}$ of the track, and the compatibility of the track originating from a pixel vertex. Tracks that pass the tightest selection are labelled highPurity. The first two iterations use pixel triplets and pixel pairs as seeds to find prompt tracks with $p_{\mathrm{T}}>0.9 \mathrm{GeV} / c$. The next iteration uses pixel triplet seeds to reconstruct low-momentum prompt tracks. The following iteration uses combinations of pixel and strip layers as seeds, and is primarily intended to find displaced tracks. The final two iterations use seeds of strip pairs to reconstruct tracks lacking pixel hits.

\section{Tracking performance}

The results presented here come from the sample described in Sect. 3, using data taken at both centre-of-mass energies $(0.9$ and $2.36 \mathrm{TeV})$, unless stated otherwise. To reduce the background from beam-gas events, discussed in Sect. 4.1.3, and to select useful events for tracking studies, two additional criteria are imposed for most of the results in this section. First, more than $20 \%$ of the reconstructed tracks in an event must be flagged as highPurity if there are at least 10 tracks in the event. Second, a primary vertex must be reconstructed in the region of pp interactions (see Sect. 6.2).

The alignment parameters for the Tracker were computed from approximately two million cosmic ray muon tracks collected during CRAFT running in November 2009 as described in Sect. 3. The nominal values of the alignment parameter errors have been used in the track reconstruction. Since the applied procedure was similar to the one discussed in Ref. [6], the resulting precision is also very similar. In particular, the width of the distribution of the mean of the residuals (taken as a measure of the local alignment precision) in the pixel barrel local $x$ and $y$ coordinates is $3 \mu \mathrm{m}$ and $4 \mu \mathrm{m}$, respectively.

The simulated events are minimum bias events produced with the PYTHIA 6.4 [29] event generator, tune D6T [30], at centre-of-mass energies of 0.9 and $2.36 \mathrm{TeV}$ (10 million events each) and processed with a simulation of the CMS detector response based on GEANT4. The misalignment, miscalibration, and dead-channel map corresponding to the detector status and calibration accuracy at the time of the first LHC collisions have been included in the simulation. The longitudinal distribution of the primary collision vertices has been adjusted to match the data.

\subsection{Basic tracking distributions}

The highPurity tracks are selected, with additional requirements of $\left|d_{z}\right|<10 \sigma_{z}$ (where $d_{z}$ is the longitudinal impact parameter with respect to the primary vertex and $\sigma_{z}$ is the combined track and primary vertex uncertainty in $z$ ) and
$\sigma_{p_{\mathrm{T}}} / p_{\mathrm{T}}<10 \%$, to compare the data and simulation. Figure 9 shows the results of this comparison for several important track parameters. The distribution of the number of tracks per event, shown in Fig. 9a, has been normalized to the number of events. The data clearly have more tracks per event than are present in the simulated data. This result, combined with other CMS results, is being used to refine the PYTHIA model used in generating simulated data in order to better match the observed data. To be able to compare shapes, the other distributions have been normalized to the number of reconstructed tracks in the data. There is general agreement between the data and simulation distribution shapes for all other tracking variables. In particular, the features in the $\phi$ distribution, due to inactive modules, are well modelled by the simulation.

\subsection{Primary vertex resolution}

The reconstruction of the primary interaction vertex in the event starts from the track collection. The tracks are clustered based on the $z$ coordinate of the track at the point of closest approach to the beamline. The clusters are fit with an adaptive vertex fit [31], where tracks in the vertex are assigned a weight between 0 and 1 based on their proximity to the common vertex.

The primary vertex resolution strongly depends on the number of tracks used in fitting the vertex and on their $p_{\mathrm{T}}$. To measure the resolution, the tracks in an event with only one vertex are randomly split into two different sets and used to independently fit the primary vertex. The distribution of the difference in the fitted vertex positions can then be used to extract the resolution by fitting a Gaussian to it and dividing $\sigma$ by $\sqrt{2}$. To examine the effect of the $p_{\mathrm{T}}$ of the tracks in the vertex, we study the resolution versus the number of tracks in the vertex for different average $p_{\mathrm{T}}$ of tracks in the vertex. Figure 10 shows the $x, y$, and $z$ resolutions for different average $p_{\mathrm{T}}$ ranges. While the resolution differs considerably depending on $p_{\mathrm{T}}$ and multiplicity, the simulation accurately reproduces the data results.

\subsection{Reconstruction of particle decays}

\subsection{1 $V^{0}$ reconstruction}

$\mathrm{V}^{0}$ particles are long-lived $(c \tau>1 \mathrm{~cm})$ neutral particles reconstructed by their decay to two charged particles ${ }^{1}: \mathrm{K}_{\mathrm{S}}^{0} \rightarrow$ $\pi^{+} \pi^{-}$and $\Lambda^{0} \rightarrow p \pi^{-}$. Reconstruction of $\mathrm{V}^{0}$ decays requires finding oppositely charged tracks that are detached from the primary vertex and form a good secondary vertex with an appropriate invariant mass. For the $\Lambda^{0}$, the lowest momentum track is assumed to be the pion. As no further

\footnotetext{
${ }^{1}$ Charge conjugate states are implied throughout the paper.
} 


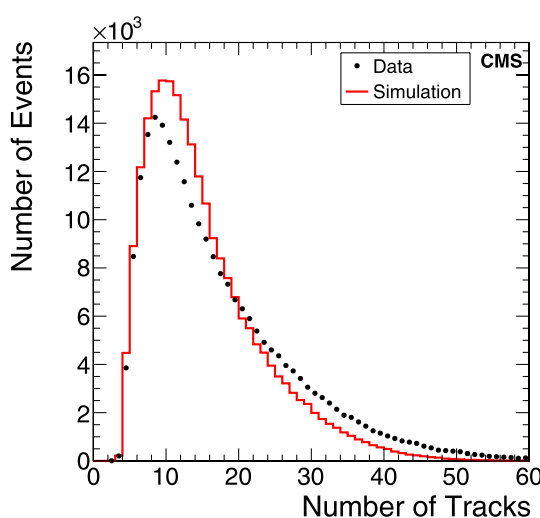

(a)

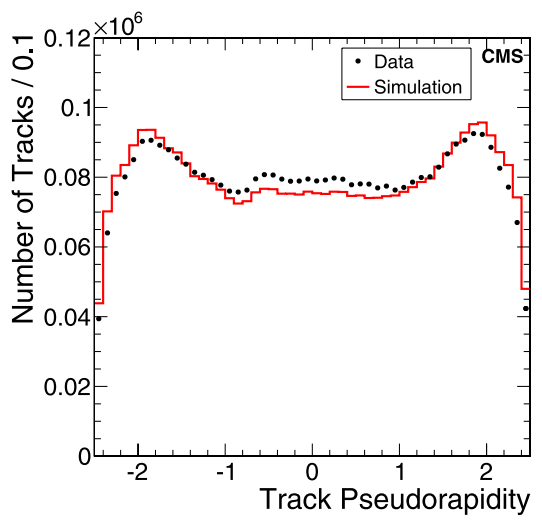

(d)

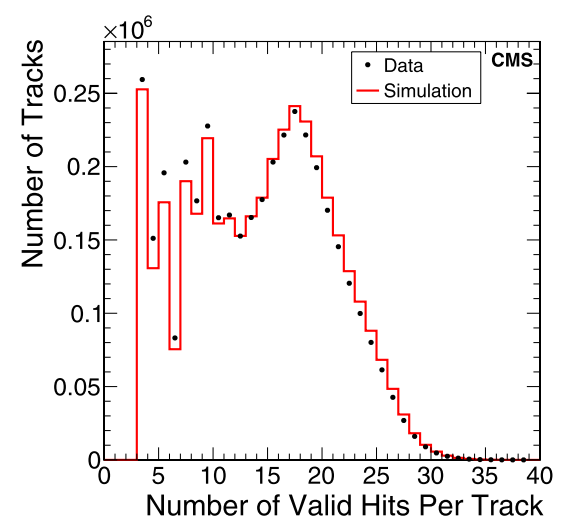

(b)

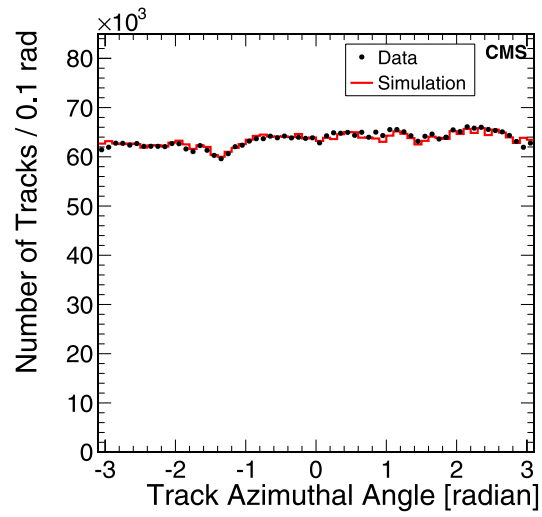

(e)

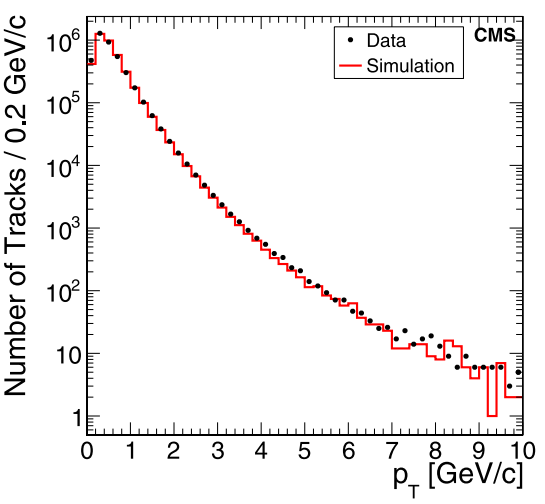

(c)

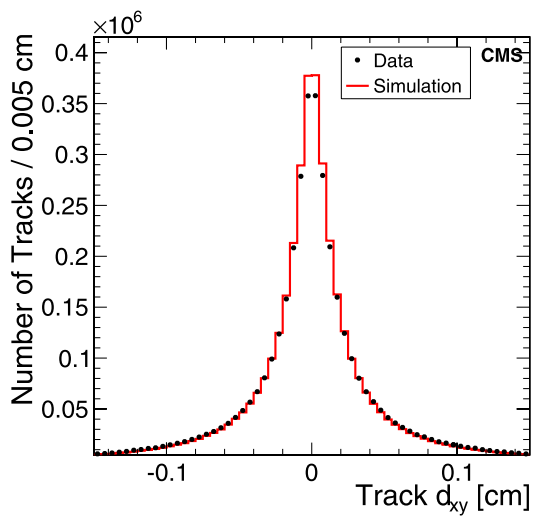

(f)

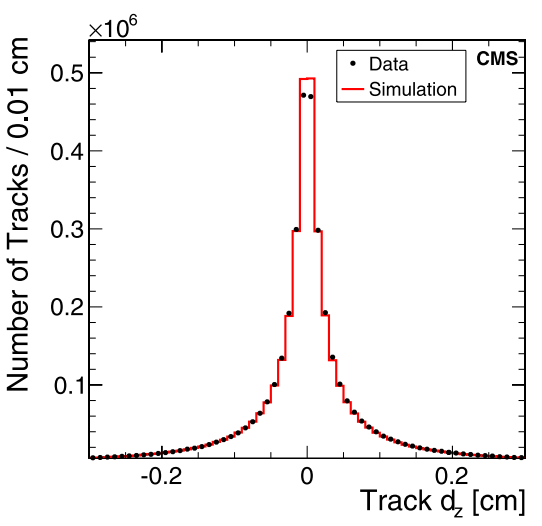

(g)

Fig. 9 Comparison of the data (points) and simulation (histogram) distributions of tracking parameters: (a) number of tracks per event, (b) number of hits used per track, transverse (c) momentum $p_{\mathrm{T}}$, (d) track pseudorapidity $\eta$, (e) azimuthal angle $\phi$, (f) transverse im-

particle identification is required, a $\mathrm{V}^{0}$ candidate can appear in both $\mathrm{K}_{\mathrm{S}}^{0}$ and $\Lambda^{0}$ samples. To be considered as a $\mathrm{V}^{0}$ decay track, a track must have at least six hits, a normalized $\chi^{2}$ less than 5, and a transverse impact parameter with respect to the beamspot greater than $0.5 \sigma_{\mathrm{IP}}$, where $\sigma_{\mathrm{IP}}$ is the calculated un-

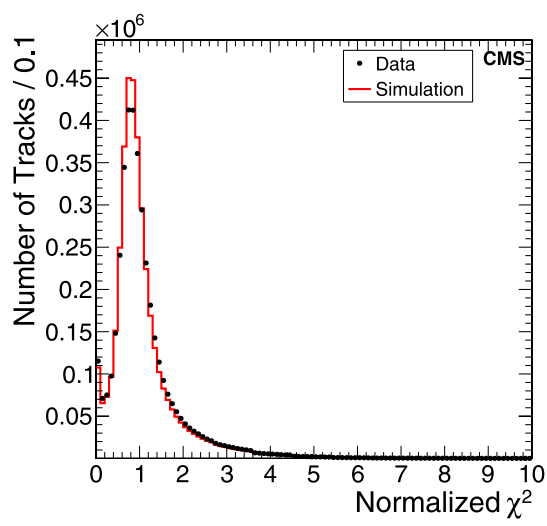

(h)

pact parameter $d_{x y}$ with respect to the primary vertex, (g) longitudinal impact parameter $d_{z}$ with respect to the primary vertex, and (h) normalized $\chi^{2}$. The simulated distributions are normalized by area to the data distributions

certainty (including beamspot and track uncertainties). The reconstructed $\mathrm{V}^{0}$ decay vertex must have a normalized $\chi^{2}$ less than 7 and a transverse separation from the beamspot greater than $15 \sigma_{T}$, where $\sigma_{T}$ is the calculated uncertainty (including beamspot and vertex uncertainties). In addition, 


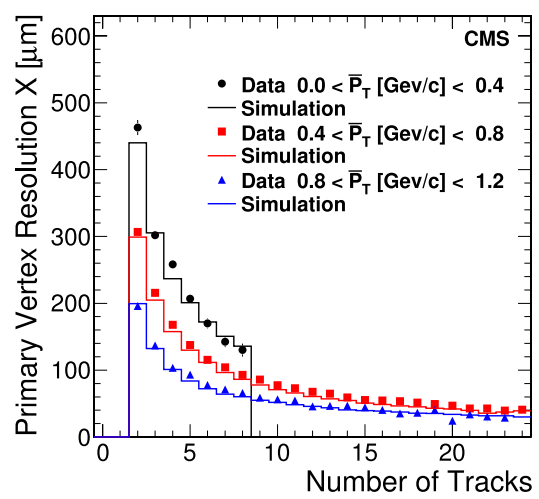

(a)

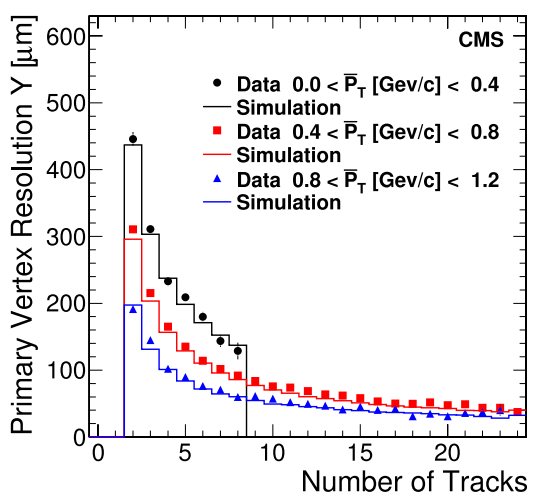

(b)

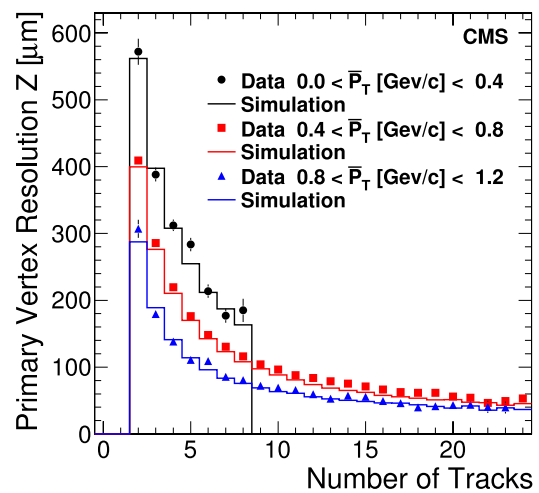

(c)

Fig. 10 Primary vertex resolution distributions in (a) $x$, (b) $y$, and (c) $z$ versus number of tracks. The three sets of results in each plot show different average $p_{\mathrm{T}}$ ranges and within each $p_{\mathrm{T}}$ range, data and simulation are compared

Table 4 Masses obtained from data, world average [32], and simulation (reconstructed and generated). The uncertainties for data and simulation results are statistical only

\begin{tabular}{|c|c|c|c|c|}
\hline \multirow[t]{2}{*}{$\mathrm{V}^{0}$} & \multicolumn{4}{|l|}{ Mass $\left(\mathrm{MeV} / c^{2}\right)$} \\
\hline & Data & PDG & Simulation & Generated \\
\hline $\mathrm{K}_{\mathrm{S}}^{0}$ & $497.68 \pm 0.06$ & $497.61 \pm 0.02$ & $498.11 \pm 0.01$ & 497.670 \\
\hline$\Lambda^{0}$ & $1115.97 \pm 0.06$ & $1115.683 \pm 0.006$ & $1115.93 \pm 0.02$ & 1115.680 \\
\hline
\end{tabular}

the $\mathrm{V}^{0}$ candidate is discarded if either of the daughter tracks has hits that are more than $4 \sigma_{3 D}$ from the $\mathrm{V}^{0}$ vertex, toward the primary vertex, where $\sigma_{3 D}$ is the uncertainty in the vertex position.

The mass resolution of the $\mathrm{V}^{0}$ depends on $\eta$ as well as on the decay vertex position and a single Gaussian is not a sufficiently accurate functional form for the signal. Therefore, a double Gaussian with the same mean was used to fit the signal. For the background shapes, a linear background was used for $\pi^{+} \pi^{-}$and the function $a\left(m-m_{p}-m_{\pi}\right)^{b}$ was used for the $p \pi^{-}$spectrum where $m$ is the $p \pi^{-}$invariant mass and $a$ and $b$ are free parameters. The $\pi^{+} \pi^{-}$ and $p \pi^{-}$mass distributions, along with the overlaid fits, are shown in Figs. 11a and 11b, respectively. Tables 4 and 5 show the reconstructed $\mathrm{V}^{0}$ masses and resolutions obtained from the data and simulation. While the various results are close to expectations, significant discrepancies are present. These features can be examined as a function of track kinematic variables to better understand the CMS tracker and magnetic field. This work is ongoing.

\subsection{2 $V^{0}$ lifetime}

For the $0.9 \mathrm{TeV}$ centre-of-mass energy data and simulation, invariant mass distributions are made for different bins of proper decay length, $c t=m c L / p$, where $L$ is the measured decay length. These distributions are fitted to obtain
Table $5 \mathrm{~V}^{0}$ mass resolutions obtained from data and simulation. The narrow and wide Gaussian resolutions are $\sigma_{1}$ and $\sigma_{2}$, respectively. The $\sigma_{1}$ fraction is the fraction of the yield from the narrow Gaussian. The final row gives the average resolution, obtained from the square root of the weighted average of the two resolutions squared. Uncertainties are statistical only

\begin{tabular}{lllll}
\hline Parameter & $\mathrm{K}_{\mathrm{S}}^{0}$ Data & $\mathrm{K}_{\mathrm{S}}^{0}$ Simulation $\Lambda^{0}$ Data & $\Lambda^{0}$ Simulation \\
\hline$\sigma_{1}\left(\mathrm{MeV} / c^{2}\right)$ & $4.53 \pm 0.12$ & $4.47 \pm 0.04$ & $1.00 \pm 0.26$ & $1.71 \pm 0.05$ \\
$\sigma_{2}\left(\mathrm{MeV} / c^{2}\right)$ & $11.09 \pm 0.41$ & $10.49 \pm 0.11$ & $3.25 \pm 0.14$ & $3.71 \pm 0.09$ \\
$\sigma_{1}$ fraction & $0.58 \pm 0.03$ & $0.58 \pm 0.01$ & $0.15 \pm 0.05$ & $0.44 \pm 0.03$ \\
$\bar{\sigma}\left(\mathrm{MeV} / c^{2}\right)$ & $7.99 \pm 0.14$ & $7.63 \pm 0.03$ & $3.01 \pm 0.08$ & $2.99 \pm 0.03$ \\
\hline
\end{tabular}

the yield, leading to the uncorrected $c t$ distribution as seen in Fig. 12a for the $\mathrm{K}_{\mathrm{S}}^{0}$ data. The uncorrected $c t$ distribution from the simulation is divided by the generated exponential shape given by $e^{-c t / c \tau_{\text {Sim }}}$ to obtain the correction factor versus $c t$. The uncorrected data $c t$ distribution is divided by the correction factor to obtain the corrected $c t$ distribution as seen in Fig. $12 \mathrm{~b}$ for the $\mathrm{K}_{\mathrm{S}}^{0}$. This distribution is fitted with an exponential, the slope of which gives the measured lifetime. The good fit to an exponential function $\left(\chi^{2} / \mathrm{NDOF}=8.1 / 8\right)$ indicates that the simulation accurately reproduces the efficiency variation versus lifetime. The fitted results, $\tau_{\mathrm{K}_{\mathrm{S}}^{0}}=90.0 \pm 2.1 \mathrm{ps}$ and $\tau_{\Lambda^{0}}=271 \pm 20 \mathrm{ps}$ (with $\chi^{2} / \mathrm{NDOF}=11.3 / 6$ ), are both within $1 \sigma$ of the world average [32].

\subsubsection{Reconstruction of $K^{*}(892)^{-}$and $\Xi^{-}$}

The reconstructed sample of $\mathrm{V}^{0}$ particles was exploited to reconstruct decays of other particles.

The $\mathrm{K}_{\mathrm{S}}^{0}$ candidates are combined with charged tracks from the primary vertex to search for the strong decay $\mathrm{K}^{*}(892)^{-} \rightarrow \mathrm{K}_{S}^{0} \pi^{-}$. For this analysis, events were required to contain a reconstructed primary vertex consisting of more 
Fig. 11 The invariant mass distributions of (a) $\pi^{+} \pi^{-}$with a fit to the $\mathrm{K}_{\mathrm{S}}^{0}$ and (b) $p \pi^{-}$with a fit to the $\Lambda^{0}$

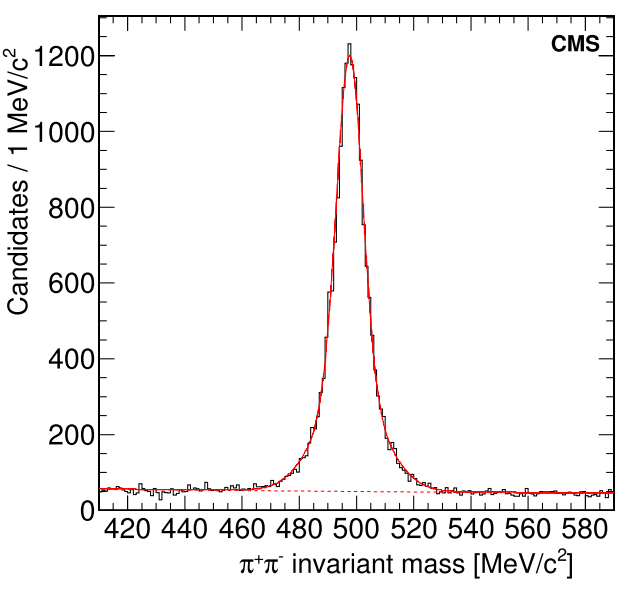

(a)

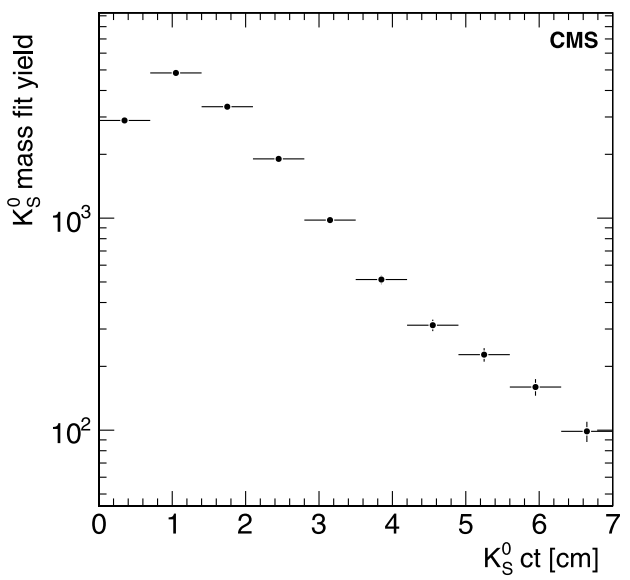

(a)

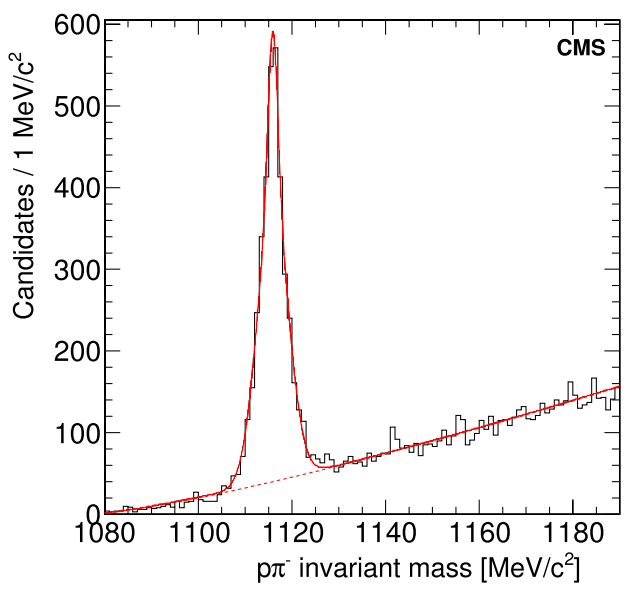

(b)

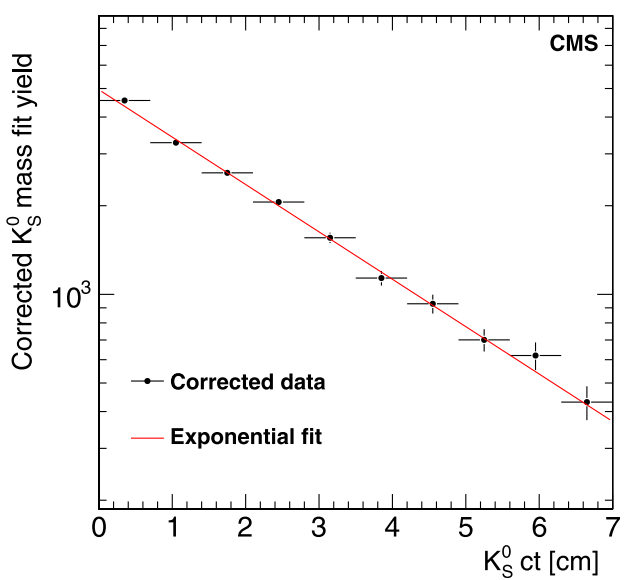

(b) than two tracks and a fit probability greater than $0.5 \%$. The $\mathrm{K}_{\mathrm{S}}^{0}$ candidate must pass the same criteria as described in Sect. 6.3.1. In addition, the requirement on the impact parameter significance of the pions from the $\mathrm{K}_{\mathrm{S}}^{0}$ is increased from 0.5 to 2 . The $\mathrm{K}_{\mathrm{S}}^{0}$ candidates must also have a mass within $20 \mathrm{MeV} / \mathrm{c}^{2}$ of the nominal mass and the $\mathrm{K}_{\mathrm{S}}^{0}$ flight path must pass within 2 mmof the primary vertex. The charged track in the $\mathrm{K}^{*}(892)^{-}$decay must have a normalized $\chi^{2}$ less than 2, at least two hits in the pixel detector, at least seven total hits, $p_{\mathrm{T}}>0.5 \mathrm{GeV} / c,|\eta|<2$, and pass within 2 (3) $\mathrm{mm}$ of the primary vertex in the direction transverse to (along) the beam line. The $\mathrm{K}_{\mathrm{S}}^{0} \pi^{-}$invariant mass is calculated using the world-average value of the $\mathrm{K}_{\mathrm{S}}^{0}$ mass [32] and is shown in Fig. 13a. The figure also shows an overlay of a fit to the $\mathrm{K}_{\mathrm{S}}^{0} \pi^{-}$mass distribution. The fit uses a Breit-Wigner for the signal plus a threshold function for the background

$$
\begin{aligned}
& \frac{S}{\left(m^{2}-M_{K^{*}}^{2}\right)^{2}+\Gamma_{K^{*}}^{2} M_{K^{*}}^{2}} \\
& +B\left[1-\exp \left(\frac{M_{K}+M_{\pi}-m}{p}\right)\right],
\end{aligned}
$$

where $m$ is the $\mathrm{K}_{\mathrm{S}}^{0} \pi^{-}$invariant mass, $M_{K^{*}}$ and $\Gamma_{K^{*}}$ are the mass and width of the $\mathrm{K}^{*}(892)^{-}, M_{K}$ and $M_{\pi}$ are the world-average masses of $K^{0}$ and $\pi^{-}$, and $S, B$, and $p$ are free parameters. The $\mathrm{K}^{*}$ width $\left(\Gamma_{K^{*}}\right)$ is fixed at the world-average value of $50.8 \mathrm{MeV} / c^{2}$ [32], while the $\mathrm{K}^{*}$ mass $\left(M_{K^{*}}\right)$ is a free parameter. The mass returned by the fit, $888.3 \pm 3.2 \mathrm{MeV} / c^{2}$, is consistent with the world-average value of $891.66 \pm 0.26 \mathrm{MeV} / c^{2}$ [32]

The $\Xi^{-}$was reconstructed through its decay to $\Lambda^{0} \pi^{-}$. The $\Xi^{-}$is a long-lived baryon, with a decay topology different from that of the $\mathrm{K}^{*}(892)^{-}$: the $\pi^{-}$from the $\Xi^{-}$decay should be detached from the primary vertex rather than originating from it. The $\Lambda^{0}$ candidates were reconstructed as described in Sect. 6.3.1 except that a looser transverse significance cut of 10 (rather than 15) was applied. $\Lambda^{0}$ candidates with a mass within $8 \mathrm{MeV} / \mathrm{c}^{2}$ of the world-average value were combined with charged tracks with the same sign as the pion in the $\Lambda^{0}$ decay. The $\Lambda^{0} \pi^{-}$fit used a $\Lambda^{0}$ mass constraint and the vertex was required to have a fit probability better than $1 \%$. All three tracks involved in the decay were required to have at least six valid hits and a 3D im- 
Fig. 13 Invariant mass plots of (a) $\mathrm{K}_{\mathrm{S}}^{0} \pi^{-}$with a fit to the $\mathrm{K}^{*}(892)^{-}$and (b) $\Lambda^{0} \pi^{-}$with a fit to the $\Xi^{-}$

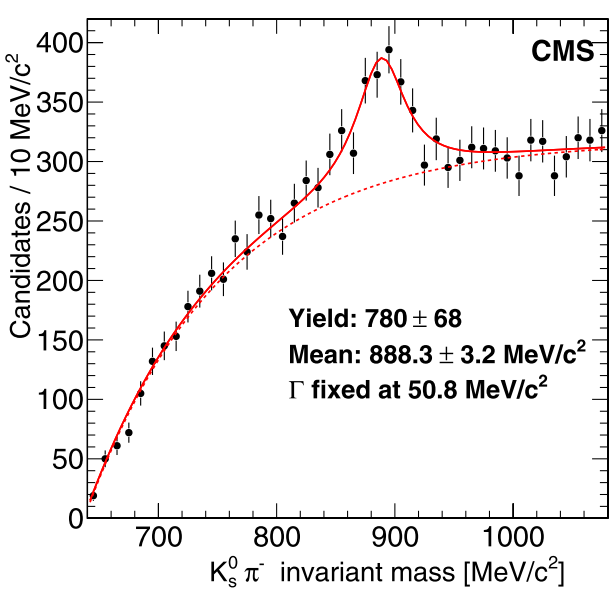

(a)

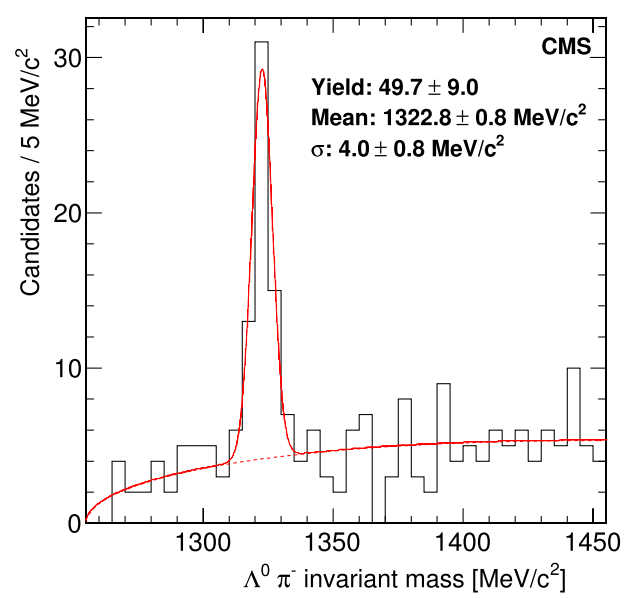

(b) pact parameter with respect to the primary vertex greater than $3 \sigma$. The resulting mass plot, shown in Fig. 13b, is fit with a single Gaussian for the signal and a background shape of $A q^{(1 / 2)}+B q^{(3 / 2)}$ where $q=m-M_{\Lambda}-M_{\pi}, m$ is the $\Lambda^{0} \pi^{-}$invariant mass, and $A$ and $B$ are free parameters. The measured mass of $1322.8 \pm 0.8 \mathrm{MeV} / c^{2}$ is close to the world-average value of $1321.71 \pm 0.07 \mathrm{MeV} / \mathrm{c}^{2}$ [32]. The resolution of $4.0 \pm 0.8 \mathrm{MeV} / \mathrm{c}^{2}$ is consistent with the simulation result of $3.6 \pm 0.4 \mathrm{MeV} / \mathrm{c}^{2}$.

\subsection{Particle identification using measured energy losses}

Estimating the energy loss $(d E / d x)$ of a particle by means of charge collected by the CMS silicon strip tracker is described in Sect. 4.2.5. In this section, applications of $d E / d x$ measurements are used to identify protons and kaons produced in $\Lambda^{0}$ and $\phi$ decays.

\subsection{1 $d E / d x$ verification with $\Lambda \rightarrow p \pi^{-}$decays}

The kinematics of the $\Lambda^{0} \rightarrow p \pi^{-}$decay requires $p_{p}>p_{\pi}$ for all $\Lambda^{0}$ particles reconstructed at CMS. This provides a clean source of protons and pions which can be used to check the $d E / d x$ results. We apply the same selection as in Sect. 6.3.1, and plot the $d E / d x$ distribution as a function of the momentum for tracks associated to $\mathrm{V}^{0}$ candidates in the mass range $1.11-1.12 \mathrm{GeV} / c^{2}$, separately for the highest momentum tracks (Fig. 14a) and the lowest momentum tracks (Fig. 14b). As expected, the highest momentum tracks are generally found near the proton curve while the lowest momentum tracks are generally inconsistent with the proton curve. The few exceptions are consistent with background under the $\Lambda^{0}$ peak.

\subsubsection{Reconstruction of $\phi(1020) \rightarrow \mathrm{K}^{+} \mathrm{K}^{-}$}

The $\phi(1020) \rightarrow \mathrm{K}^{+} \mathrm{K}^{-}$decay was reconstructed using data taken at $0.9 \mathrm{TeV}$ centre-of-mass energy. The candidate kaon tracks come from the collection of highPurity tracks and are required to have $p_{\mathrm{T}}>0.5 \mathrm{GeV} / c$, normalized $\chi^{2}<2$, at least five hits, $|\eta|<2$, and a transverse impact parameter with respect to the reconstructed beamspot smaller than $3 \mathrm{~mm}$. Finally, for tracks with $p<1 \mathrm{GeV} / c$, the track must have a measured $d E / d x$ consistent with the kaon hypothesis $(\operatorname{see}(2)): K\left(M^{\min } / p\right)^{2}+C<d E / d x<K\left(M^{\max } / p\right)^{2}+C$. The parameters of the $d E / d x$ cut for kaons are those extracted from a fit to the $d E / d x$ vs. $p$ distribution, as described in Sect. 4.2.5. We use a compatibility window of $\pm 200 \mathrm{MeV} / c^{2}$ around the $\mathrm{K}$ mass, with $M^{\min }$ and $M^{\max }$ being lower and upper boundaries of this window.

The fit of the mass spectra of pairs of tracks accepted by the $d E / d x$ selection used the sum of two normalized functions: a convolution of a relativistic Breit-Wigner shape with a Gaussian for the $\phi$ signal and an arctangent function for the background. The mass plot and overlaid fit are shown in Fig. 15a. The fitted $\phi$ mass of $1019.58 \pm$ $0.22 \mathrm{MeV} / \mathrm{c}^{2}$ is in agreement with the world-average value of $1019.455 \pm 0.020 \mathrm{MeV} / c^{2}$. The resolution found in data is $1.29 \pm 0.32 \mathrm{MeV} / c^{2}$, in agreement with the value found in simulation, $1.41 \mathrm{MeV} / \mathrm{c}^{2}$. Candidates in which at least one track fails the $d E / d x$ requirement are shown in Fig. 15b where only background is observed, indicating that the $d E / d x$ requirement has a high efficiency to select $\phi(1020)$ candidates.

\subsection{Reconstruction of photon conversions and nuclear interactions}

While the tracker is essential for finding charged particles and measuring their momenta, the tracker material is also a source for interactions. For photons, interactions with the tracker material can produce $e^{+} e^{-}$conversion pairs, while for hadrons, nuclear interactions can produce multiple hadrons. Photon conversions in the Tracker reduce the 
Fig. 14 Estimated energy loss as a function of the momentum for (a) the highest momentum track and (b) the lowest momentum track for the $\Lambda^{0}$ candidate decay products. The superimposed curves comes from the proton fit in the inclusive track sample shown in Fig. 8a

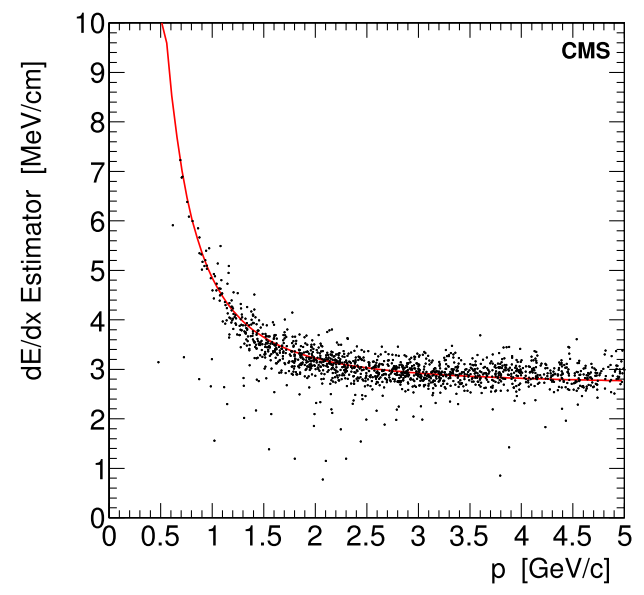

(a)

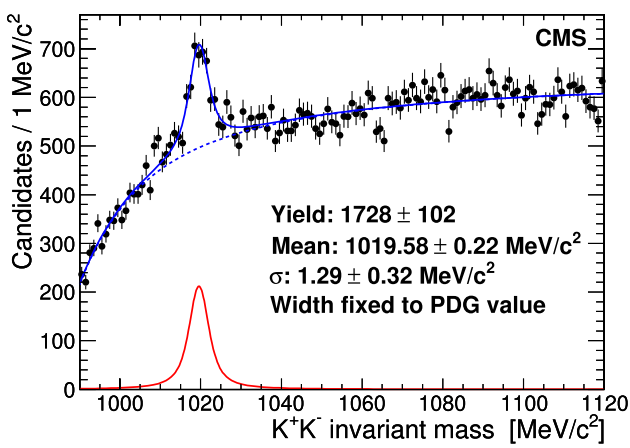

(a)

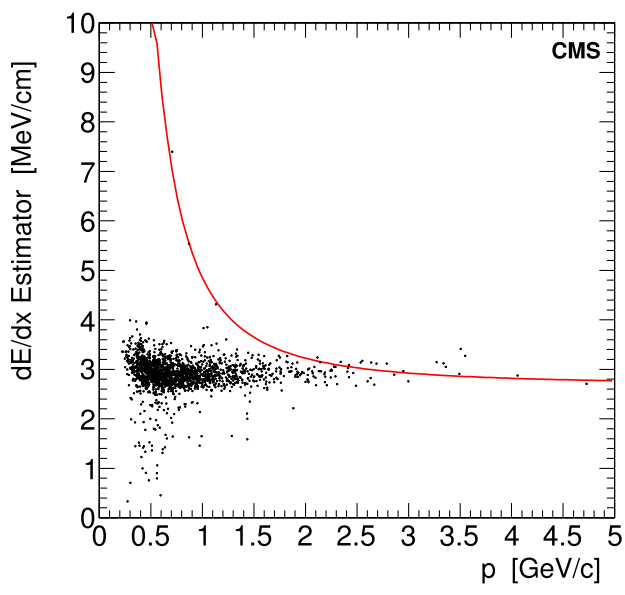

(b)

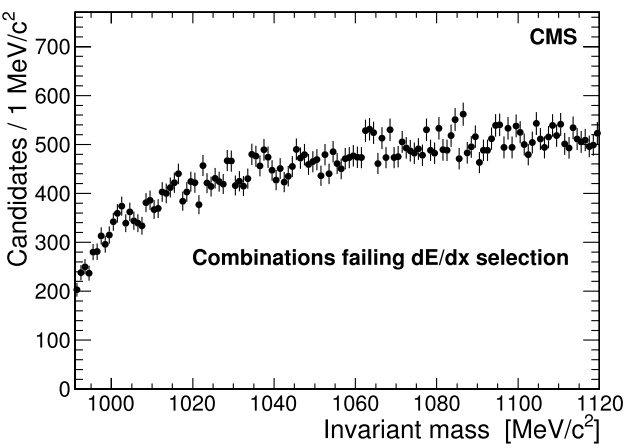

(b) efficiency for low-energy-photon finding by the electromagnetic calorimeter, while nuclear interactions reduce track finding efficiency and can affect the resolution of many hadronic observables such as jets or missing transverse energy. Thus, identification of conversions and nuclear interactions can be used to improve many aspects of the event reconstruction. Furthermore, studies of conversions and interactions can be used to improve our understanding of the material in the Tracker.

The electrons and positrons from converted photons can be identified by the electromagnetic calorimeter and used as seeds for track reconstruction [33]. In the minimum bias events collected in December 2009, however, the photons have a soft spectrum as seen in Fig. 16a and therefore the conversion pairs are unlikely to reach the electromagnetic calorimeter. These conversion pairs can still be reconstructed by using tracker-seeded conversion reconstruction techniques, made possible by the iterative tracking algorithm described in Sect. 5 which extends the capability of reconstructing low- $p_{\mathrm{T}}$ and detached tracks. The essential signature of a massless conversion photon is the two parallel tracks at the production vertex, in both the transverse and longitudinal planes. The reconstructed invariant mass, shown in Fig. 16b, shows the effect of the mass res- olution, which is well modelled by the simulation. Two different conversion reconstruction approaches have been used. Both methods fit two oppositely charged tracks to a common $3 \mathrm{D}$ vertex with the constraint that the two tracks are parallel at the vertex. The methods differ mainly in the preselection of the track pairs. The first method, from which Figs. 16a and $16 \mathrm{~b}$ are derived, requires both tracks have at least three hits and normalized $\chi^{2}$ less than 10 and at least one track with five or more hits. The tracks are required to have positive charge-signed transverse impact parameter, positive distance of minimum approach in 2D (i.e., the two full track circles have one or no intersection in the transverse plane), small $z$ separation at their innermost point $(|\Delta z|<5 \mathrm{~cm})$ if they are in the barrel, and a small opening angle in both the transverse $(\Delta \phi<0.2)$ and longitudinal plane $(\Delta \cot \theta<0.1$ where $\theta$ is the polar angle relative to the $z$ axis). The vertex fit must have a $\chi^{2}$ probability better than $5 \times 10^{-3}$ and be located inside the innermost hits on the tracks. To increase efficiency, the second method takes all tracks with a $\chi^{2}$ probability above $10^{-6}$ and requires a vertex with fit probability greater than $10^{-6}$, radius greater than $2 \mathrm{~cm}$, and at most one hit per track inside of the vertex position. The $\chi^{2}$ probability from the second method is shown in Fig. 16c with good agreement between data and simulation. 


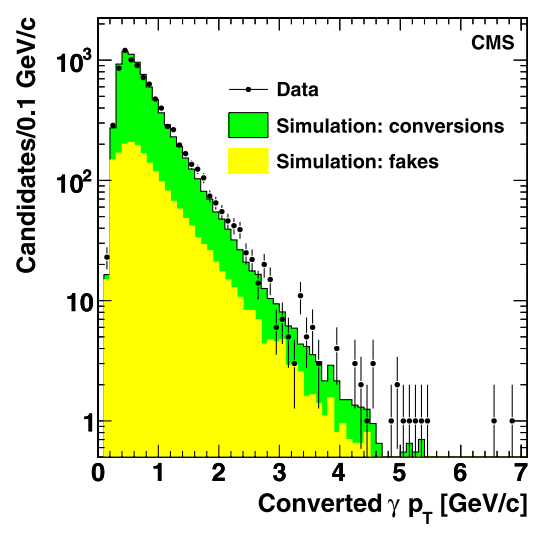

(a)

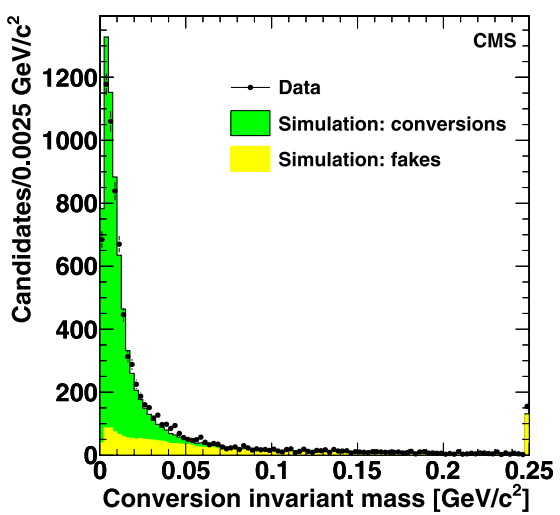

(b)

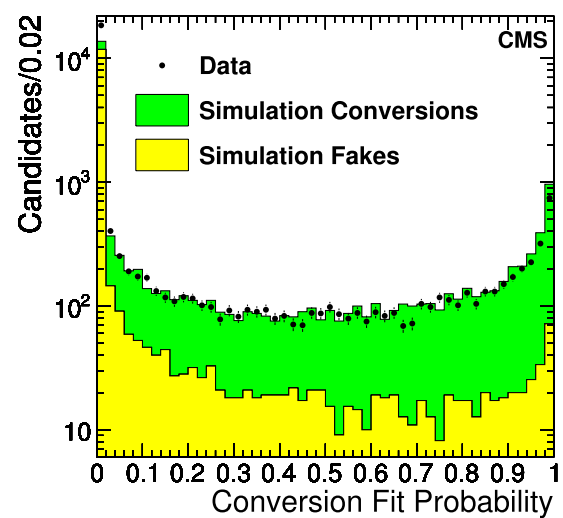

(c)
Fig. 16 Comparisons of data photon conversions (points) and real and fake photon conversion from simulation (filled histograms) showing: (a) distributions of the reconstructed $p_{\mathrm{T}}$ of the converted photons from the first method, (b) the invariant mass of the $e^{+} e^{-}$pairs from the first method, and (c) the distribution of the vertex $\chi^{2}$ probability from the second method. The last bin of (b) is the overflow bin
The nuclear interaction finder starts from the full list of tracks described in Sect. 5. For each pair of tracks, the distance of closest approach is computed and if the two tracks are close enough they are considered linked together. A recursive finder produces blocks of tracks linked together from which a rough estimate of the displaced vertex position is computed. Finally, the tracks from a block are refitted together with a displaced vertex as a common constraint. $V^{0}$ decays and photon conversions are removed from the resulting sample of displaced vertices. A tight selection is applied to the remaining vertices to remove fake tracks and pairs from the primary vertex. The resulting sample of significantly displaced vertices in the radial direction $(r>2.5 \mathrm{~cm})$ is called the nuclear interactions sample. In the data, $80 \%$ of nuclear interactions are reconstructed with two tracks and $20 \%$ with three tracks. In the first case, a $30 \%$ combinatorial fake rate is expected from the simulation, while in the second case the fake rate is negligible.

The distribution of nuclear interaction positions provides a means of observing the material in the detector and validating the simulation of the material. The distribution of radial position $r$ of the nuclear vertices, compared to the simulation, is shown in Fig. 17a. The beam pipe at a radius of $3 \mathrm{~cm}$, as well as the three barrel pixel layers at average radii of $4.3,7.3$, and $10.2 \mathrm{~cm}$, are clearly seen. The radius is measured relative to the centre of the pixel detector. In the version of the simulation used here, this is also the centre of the beam pipe. In reality, the beam pipe centre is offset from the pixel detector centre, resulting in a smeared distribution versus radius. Nevertheless, there is good agreement between the data and the simulation for the relative rate of nuclear interactions in the different barrel pixel structures and the beam pipe. This indicates a consistent description of the material distribution in this region. The material distribution in the endcap pixel detector is studied by selecting nuclear interactions with $|z|>26 \mathrm{~cm}$ and $r<19 \mathrm{~cm}$. The longitudinal position $|z|$ of the nuclear vertices, compared to the simulation, is shown in Fig. 17b. The pixel barrel flange $(|z|<30 \mathrm{~cm})$ and the two pixel disks can be clearly distinguished. The remaining material is due to pixel services including power and data cables, cooling lines and manifolds, interface boards, and support structures.

\subsection{Study of $b$-tag related observables}

The measurement of impact parameters and the reconstruction of secondary vertices, the main ingredients in b-tagging algorithms, have been tested with the limited event sample of December 2009. The measurements of track impact parameter and secondary vertex separation take advantage of relatively long lifetime of $b$ hadrons to identify $b$ jets.

Both impact parameter and vertex based $b$-jet identification algorithms [34, 35] start by associating tracks to a jet. Jet reconstruction is performed using the anti- $k_{\mathrm{T}}$ jet clustering algorithm $[36,37]$ on objects obtained by combining the information from tracks with energy deposits in the electromagnetic and hadron calorimeters [38, 39]. The track association uses the parameter $\Delta R=\sqrt{(\Delta \phi)^{2}+(\Delta \eta)^{2}}$, which defines a cone around the jet axis, to determine if a track is part of the jet. The tracks must also pass basic quality criteria based on the transverse momentum, the number of pixel and strip hits associated to the track, and the $\chi^{2}$ of the track fit. To reject badly measured tracks and products of interactions with material or decays of long-lived strange particles, tracks must pass loose requirements on the transverse and longitudinal impact parameters (with respect to the primary vertex). Using the point of closest approach between the track and the jet axis, distances to the jet axis and the primary vertex are measured and criteria applied to reduce contributions from possible additional interaction points. 
Fig. 17 Distributions of nuclear interaction vertices versus (a) radial position $r$ for $|z|<26 \mathrm{~cm}$ and (b) versus the magnitude of the longitudinal coordinate $|z|$ for $|z|>26 \mathrm{~cm}$ and $r<19 \mathrm{~cm}$. The simulation histogram is normalized to the total number of nuclear interactions found in data in the full $z$ range

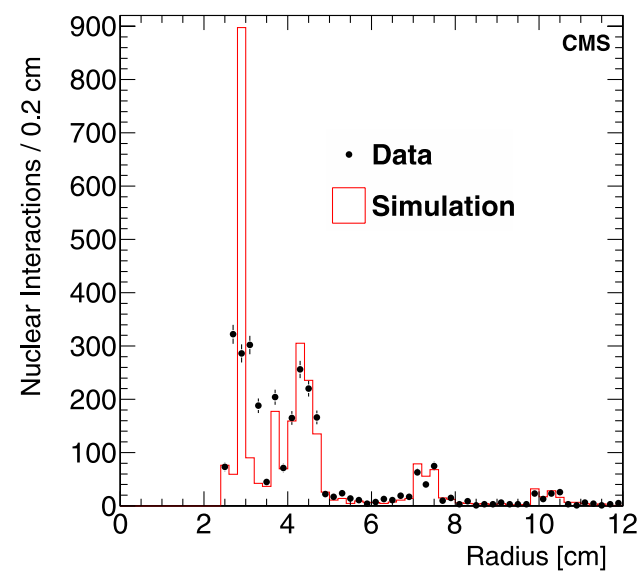

(a)

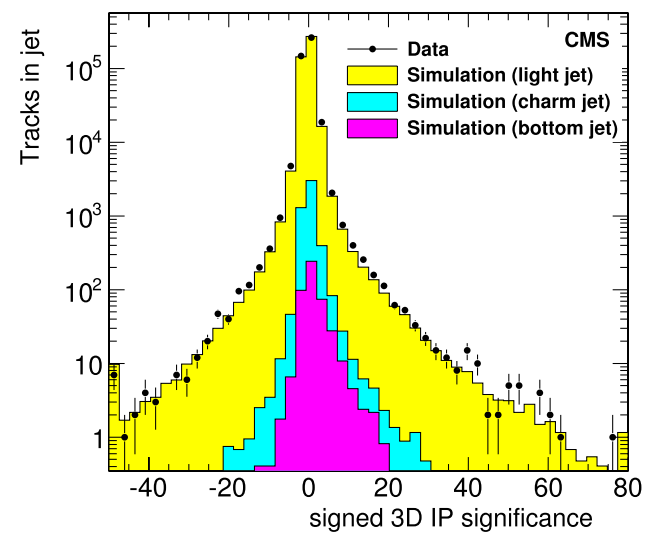

(a)

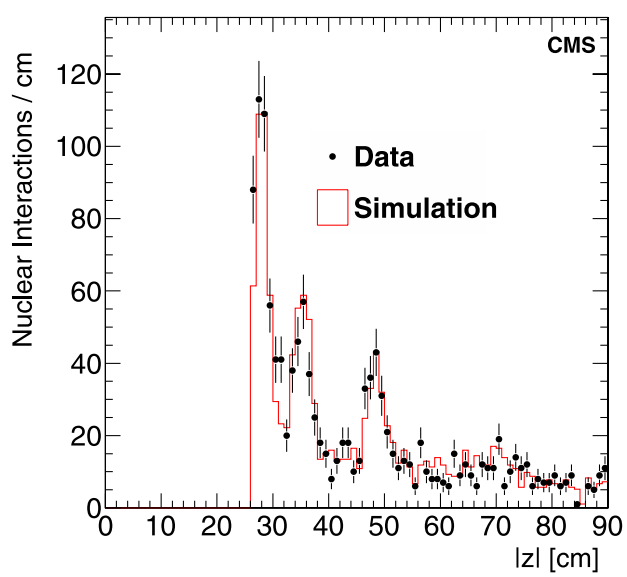

(b)

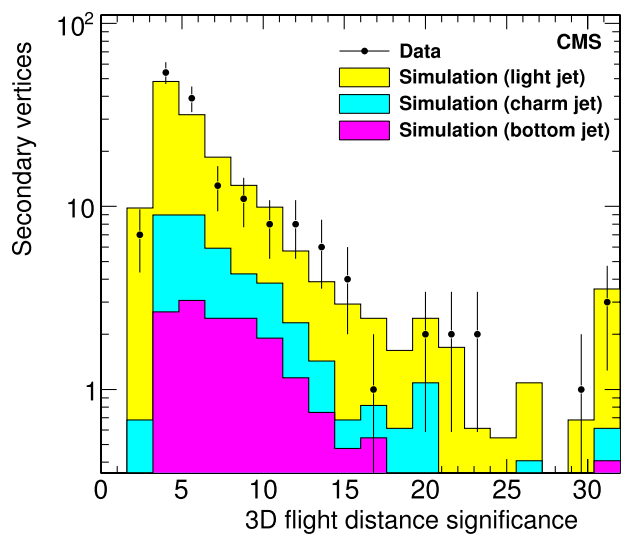

(b)
The 2009 data contain only a few well-defined jets and mainly tracks at momenta below those typically used in $b$ tagging. To test the reconstruction on a sufficiently large sample, requirements are relaxed compared to typical selections at higher energies $[34,35]$. The jet clustering and track association use $\Delta R<0.7$ and a minimum jet $p_{T}$ of $3 \mathrm{GeV} / c$. The tracks are required to have a minimum of seven hits and are not subject to a minimum $p_{\mathrm{T}}$ requirement. The selected tracks are used to calculate three-dimensional impact parameters with respect to the reconstructed primary vertex. The impact parameters are "lifetime signed": tracks with an apparent production point in the direction of the jet axis are assigned a positive sign. Figure 18a shows the threedimensional impact parameter significance distribution for all tracks associated with jets. The data show good agreement with the results from a minimum bias simulation reconstructed with the same algorithm settings.

To limit the number of vertex combinations, slightly stricter track quality requirements are used to select the tracks associated with secondary vertices. The secondary vertex reconstruction uses the adaptive vertex fitter in an iterative way on the tracks rejected in the previous itera- tions [31]. Vertex candidates must have at least two tracks, not be compatible with the $\mathrm{K}_{\mathrm{S}}^{0}$ mass, and share less than $65 \%$ of their tracks with the primary vertex. Vertices must have a transverse separation from the primary vertex of at least $100 \mu \mathrm{m}$ and $3 \sigma$, but no more than $2.5 \mathrm{~cm}$. Badly reconstructed vertices are rejected by a $\Delta R<1$ cut between the flight direction of the secondary vertex and the jet axis as well as the requirement that the invariant mass be less than $6.5 \mathrm{GeV} / \mathrm{c}^{2}$. Figure $18 \mathrm{~b}$ shows the significance of the distance between primary and secondary vertices compared to what is expected from a simulation of minimum bias events. While many two- and three-track vertices are reconstructed, only one four-track vertex is found in the data. This event is shown in Fig. 19.

\section{Conclusion}

The all-silicon CMS Tracker was designed for LHC physics. During its conception, emphasis was placed on providing fine granularity, large pseudorapidity coverage, and a number of redundant measurements to facilitate the pattern 


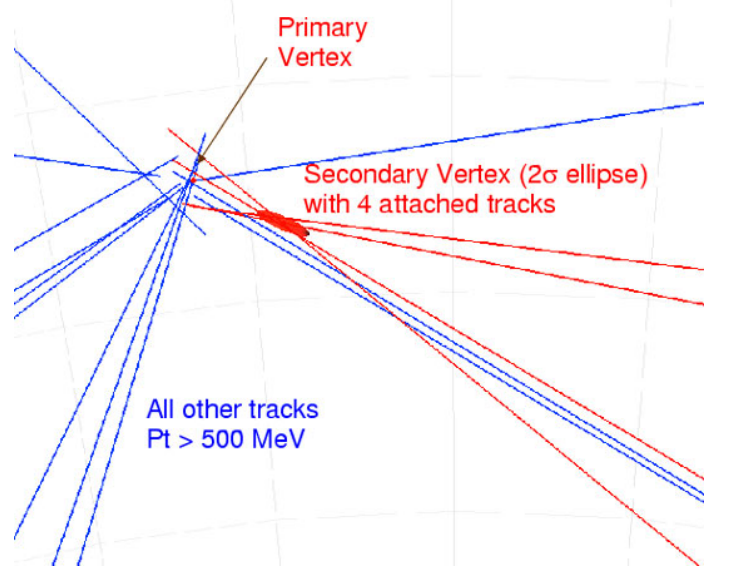

Fig. 19 Display of an event with a four-track secondary vertex. The vertex is separated from the primary vertex by $2.6 \mathrm{~mm}$, corresponding to $7 \sigma$, and the invariant mass of the four particles is $1.64 \mathrm{GeV} / c^{2}$, assuming they are all pions

recognition. Meeting these design criteria has resulted in excellent tracking performance for the large-volume detector operating in a 3.8 T magnetic field in the early collision running. The proximity of the pixel detector to the LHC beam line permits precise reconstruction of primary and secondary vertices. The extended commissioning with cosmic rays in 2008 and 2009 allowed most calibrations to be completed and provided a good initial alignment for most of the detector. This allowed fast and reliable operation of the Tracker in the first LHC collisions in December 2009.

The performance of the Tracker has been studied using the collision data at centre-of-mass energies of 0.9 and $2.36 \mathrm{TeV}$. The tracking and vertexing resolutions are in agreement with the expected design performance in minimum bias events, for the level of the alignment achieved. Studies of the decays of $\mathrm{K}_{\mathrm{S}}^{0}, \Lambda^{0}, \Xi^{-}$, and $\mathrm{K}^{*}(892)^{-}$test the capability to reconstruct displaced vertices and agree well with predictions from simulation. In particular, measurements of $\mathrm{V}^{0}$ mass, resolution, and lifetime provide strong tests of our understanding of the magnetic field, Tracker material, and detector performance. Our knowledge of the Tracker material is also evident in the agreement between data and simulation for photon conversions and nuclear interactions. Energy loss measurements in the Tracker, made possible by the calibration of the silicon strip analogue readout chain, provide good particle identification at low momentum as seen in the reconstructed $\phi$ and $\Lambda$ decays. Finally, the alignment parameters determined in the cosmic ray studies are already adequate for the precise determination of impact parameters and the reconstruction of secondary vertices. This will ultimately be exploited for bhadron physics and top-quark studies.

In conclusion, the CMS Tracker has been commissioned to take data at the LHC. New collision data will allow more precise alignment and calibration, which will enable the study of the new vista of particle physics that is now opening up at the LHC.

Acknowledgements We wish to congratulate our colleagues in the CERN accelerator departments for the excellent performance of the LHC machine. We thank the technical and administrative staff at CERN and other CMS institutes for their devoted efforts during the design, construction and operation of CMS. The cost of the detectors, computing infrastructure, data acquisition and all other systems without which CMS would not be able to operate was supported by the financing agencies involved in the experiment. We are particularly indebted to: the Austrian Federal Ministry of Science and Research; the Belgium Fonds de la Recherche Scientifique, and Fonds voor Wetenschappelijk Onderzoek; the Brazilian Funding Agencies (CNPq, CAPES, FAPERJ, and FAPESP); the Bulgarian Ministry of Education and Science; CERN; the Chinese Academy of Sciences, Ministry of Science and Technology, and National Natural Science Foundation of China; the Colombian Funding Agency (COLCIENCIAS); the Croatian Ministry of Science, Education and Sport; the Research Promotion Foundation, Cyprus; the Estonian Academy of Sciences and NICPB; the Academy of Finland, Finnish Ministry of Education, and Helsinki Institute of Physics; the Institut National de Physique Nucléaire et de Physique des Particules/CNRS, and Commissariat à l'Énergie Atomique, France; the Bundesministerium für Bildung und Forschung, Deutsche Forschungsgemeinschaft, and HelmholtzGemeinschaft Deutscher Forschungszentren, Germany; the General Secretariat for Research and Technology, Greece; the National Scientific Research Foundation, and National Office for Research and Technology, Hungary; the Department of Atomic Energy, and Department of Science and Technology, India; the Institute for Studies in Theoretical Physics and Mathematics, Iran; the Science Foundation, Ireland; the Istituto Nazionale di Fisica Nucleare, Italy; the Korean Ministry of Education, Science and Technology and the World Class University program of NRF, Korea; the Lithuanian Academy of Sciences; the Mexican Funding Agencies (CINVESTAV, CONACYT, SEP, and UASLP-FAI); the Pakistan Atomic Energy Commission; the State Commission for Scientific Research, Poland; the Fundação para a Ciência e a Tecnologia, Portugal; JINR (Armenia, Belarus, Georgia, Ukraine, Uzbekistan); the Ministry of Science and Technologies of the Russian Federation, and Russian Ministry of Atomic Energy; the Ministry of Science and Technological Development of Serbia; the Ministerio de Ciencia e Innovación, and Programa Consolider-Ingenio 2010, Spain; the Swiss Funding Agencies (ETH Board, ETH Zurich, PSI, SNF, UniZH, Canton Zurich, and SER); the National Science Council, Taipei; the Scientific and Technical Research Council of Turkey, and Turkish Atomic Energy Authority; the Science and Technology Facilities Council, UK; the US Department of Energy, and the US National Science Foundation.

Individuals have received support from the Marie-Curie IEF program (European Union); the Leventis Foundation; the A.P. Sloan Foundation; the Alexander von Humboldt Foundation; the Associazione per lo Sviluppo Scientifico e Tecnologico del Piemonte (Italy); the Belgian Federal Science Policy Office; the Fonds pour la Formation à la Recherche dans l'índustrie et dans l'Ágriculture (FRIA-Belgium); and the Agentschap voor Innovatie door Wetenschap en Technologie (IWTBelgium).

Open Access This article is distributed under the terms of the Creative Commons Attribution Noncommercial License which permits any noncommercial use, distribution, and reproduction in any medium, provided the original author(s) and source are credited. 


\section{References}

1. CMS Collaboration, The CMS experiment at the CERN LHC. J. Instrum. 3, S08004 (2008). doi:10.1088/1748-0221/3/08/ S08004

2. CMS Collaboration, Transverse momentum and pseudorapidity distributions of charged hadrons in pp collisions at $\sqrt{s}=0.9$ and 2.36 TeV. J. High Energy Phys. 02, 041 (2010). arXiv:1002.0621. doi:10.1007/JHEP02(2010)041

3. CMS Collaboration, Commissioning of the CMS experiment and the cosmic run at four tesla. J. Instrum. 5, T03001 (2010). arXiv:0911.4845. doi:10.1088/1748-0221/5/03/T03001

4. CMS Collaboration, Commissioning and performance of the CMS pixel tracker with cosmic ray muons. J. Instrum. 5, T03007 (2010). arXiv:0911.5434. doi:10.1088/1748-0221/5/03/T03007

5. CMS Collaboration, Commissioning and performance of the CMS silicon strip tracker with cosmic ray muons. J. Instrum. 5, T03008 (2010). arXiv:0911.4996. doi:10.1088/1748-0221/5/03/ T03008

6. CMS Collaboration, Alignment of the CMS silicon tracker during commissioning with cosmic rays. J. Instrum. 5, T03009 (2010). arXiv:0910.2505. doi:10.1088/1748-0221/5/03/T03009

7. CMS Collaboration, Precise mapping of the magnetic field in the CMS barrel yoke using cosmic rays. J. Instrum. 5, T03021 (2010). arXiv:0910.5530. doi:10.1088/1748-0221/5/03/T03021

8. Y. Allkofer et al., Design and performance of the silicon sensors for the CMS barrel pixel detector. Nucl. Instrum. Methods A 584, 25-41 (2008). arXiv:physics/0702092. doi:10.1016/j.nima.2007. 08.151

9. K. Arndt et al., Silicon sensors development for the CMS pixel system. Nucl. Instrum. Methods A 511, 106-111 (2003). doi:10. 1016/S0168-9002(03)01773-X

10. V. Chiochia et al., Simulation of heavily irradiated silicon pixel sensors and comparison with test beam measurements. IEEE Trans. Nucl. Sci. 52, 1067-1075 (2005). arXiv:physics/0411143. doi:10.1109/TNS.2005.852748

11. M. Swartz et al., Observation, modeling, and temperature dependence of doubly peaked electric fields in irradiated silicon pixel sensors. Nucl. Instrum. Methods A 565, 212-220 (2006). arXiv: physics/0510040. doi:10.1016/j.nima.2006.05.002

12. A. Moraes, C. Buttar, I. Dawson, Prediction for minimum bias and the underlying event at LHC energies. Eur. Phys. J. C 50, 435-466 (2007). doi:10.1140/epjc/s10052-007-0239-1

13. S. Cucciarelli, D. Kotlinski, T. Todorov, Position determination of the pixel hits. CMS Note CMS-NOTE-2002-049 (2002)

14. M. Swartz et al., A new technique for the reconstruction, validation, and simulation of hits in the CMS Pixel Detector. CMS Note CMS-NOTE-2007-033 (2007)

15. A. Dorokhov et al., Tests of silicon sensors for the CMS pixel detector. Nucl. Instrum. Methods A 530, 71-76 (2004). arXiv: physics/0311050. doi:10.1016/j.nima.2004.05.050

16. L. Wilke, V. Chiochia, T. Speer, Lorentz angle calibration for the barrel pixel detector. CMS Note 2008/012 (2008)

17. CMS Tracker Collaboration, Stand-alone cosmic muon reconstruction before installation of the CMS silicon strip tracker. J. Instrum. 4, P05004 (2009). arXiv:0902.1860. doi:10.1088/17480221/4/05/P05004
18. J. Agram et al., The silicon sensors for the compact muon solenoid tracker: design and qualification procedure. Nucl. Instrum. Methods A 517, 77-93 (2004). doi:10.1016/j.nima.2003.08.175

19. M. French et al., Design and results from the APV25, a deep sub-micron CMOS front-end chip for the CMS Tracker. Nucl. Instrum. Methods A 466, 359-365 (2001). doi:10.1016/S01689002(01)00589-7

20. CMS Collaboration, The CMS tracker system project: technical design report. CERN LHCC CERN-LHCC-98-006 (1998)

21. CMS Collaboration, The CMS tracker: addendum to the technical design report. CERN LHCC CERN-LHCC-2000-016 (2000)

22. S. Gadomski et al., The deconvolution method of fast pulse shaping at hadron colliders. Nucl. Instrum. Methods A 320, 217-227 (1992)

23. C. Delaere et al., Procedure for the fine delay adjustment of the CMS tracker. CMS Note CMS-NOTE-2008-007 (2008)

24. C. Eklund et al., Silicon beam telescope for CMS detector tests. Nucl. Instrum. Methods A 430, 321-332 (1999). doi:10.1016/ S0168-9002(99)00210-7

25. A. Giammanco, Particle identification with energy loss in the CMS silicon strip tracker. CMS Note CMS-NOTE-2008-005 (2008)

26. J. Allison et al., Geant 4 developments and applications. IEEE Trans. Nucl. Sci. 53(1), 270-278 (2006). doi:10.1109/TNS.2006. 869826

27. T. Miao et al., Beam position determination using tracks. CMS Note CMS-NOTE-2007-021 (2007)

28. W. Adam et al., Track reconstruction in the CMS tracker. CMS Note CMS-NOTE-2006-041 (2006)

29. T. Sjöstrand et al., PYTHIA 6.4 physics and manual. J. High Energy Phys. 05, 026 (2006). arXiv:hep-ph/0603175. doi:10.1088/ 1126-6708/2006/05/026

30. P. Bartalini, L. Fanó (eds.), Multiple Parton Interactions at the LHC, Proceedings, 1st Workshop, Perugia, Italy, October 27-31, 2008. DESY-PROC-2009-06

31. R. Frühwirth, W. Waltenberger, P. Vanlaer, Adaptive vertex fitting. CMS Note CMS-NOTE-2007-008 (2007)

32. Particle Data Group Collaboration, Review of particle physics. Phys. Lett. B 667, 1 (2008). doi:10.1016/j.physletb.2008.07.018

33. N. Marinelli, Track finding and identification of converted photons. CMS Note CMS-NOTE-2006-005 (2006)

34. CMS Collaboration, Algorithms for b Jet identification in CMS. CMS Physics Analysis Summary CMS-PAS-BTV-09-001 (2009)

35. CMS Collaboration, CMS physics: Technical design report. CERN LHCC CERN-LHCC-2006-001 (2006)

36. CMS Collaboration, Jets in 0.9 and $2.36 \mathrm{TeV}$ pp collisions. CMS Physics Analysis Summary CMS-PAS-JME-10-001 (2010)

37. M. Cacciari, G.P. Salam, G. Soyez, The anti- $k_{t}$ jet clustering algorithm. J. High Energy Phys. 04, 063 (2008). arXiv:0802.1189. doi:10.1088/1126-6708/2008/04/063

38. CMS Collaboration, Particle-flow event reconstruction in CMS and performance for jets, taus, and $E_{\mathrm{T}}^{\mathrm{miss}}$. CMS Physics Analysis Summary CMS-PAS-PFT-09-001 (2009)

39. CMS Collaboration, Commissioning of the particle-flow event reconstruction with the first LHC collisions recorded in the CMS detector. CMS Physics Analysis Summary CMS-PAS-PFT-10-001 (2010) 